\title{
Jeju-do earthworms (Oligochaeta: Megadrilacea)-Quelpart Island revisited
}

\author{
Robert J. Blakemore* \\ College of Natural Science, Hanyang University, Seoul 133-791, Korea \\ *Correspondent: rob.blakemore@gmail.com
}

\begin{abstract}
Surveys on Jeju-do (Quelpart Isl.) unearthed about 40 earthworm species or sub-species. Several considered new to science are described. Only a dozen were previously known and these are taxonomically reviewed. The two most commonly recorded in surveys by S. Kobayashi in the 1930s were Drawida anchingiana Chen, 1933 and Metaphire quelparta (Kobayashi, 1937), neither recently relocated. Morphologically similar taxa, supported with DNA barcodes, for Moniligastridae, are Drawida anchingiana seogwipo, D. anchingiana halla and D. iucn spp. or sub-spp. nov. For Megascolecidae, new taxa and synonyms are: Amynthas arx and A. aucklandis spp. nov. that have seminal grooves rather than the eversible male pores of Metaphire plus Amynthas simplex is another new species. Amynthas sangumburi Hong \& Kim, 2002 is recognized as a probable new synonym within the A. corticis (Kinberg, 1867) species-group while Amynthas corticis saeseum sub-sp nov. is described. Amynthas gracilis (Kinberg, 1867) is a new record from Jeju and Korea (with A. bouchei, A. omodeoi and A. edwardsi all by Zhao \& Qiu, 2009 possible synonyms of this species group from China) with a Jejuan sub-species, A. gracilis insularum, sub-sp. nov. Presence on Jeju of Amynthas carnosus (Goto \& Hatai, 1899) is confirmed (its further new synonyms are Chinese A. fuscus Qiu \& Sun, 2012 and A. taiwumontis Shen et al., 2013), as is A. micronarius (Goto \& Hatai, 1898) (with new synonym A. montanus Qiu \& Sun, 2012 also an invalid secondary homonym). Amynthas phaselus maculosus (Hatai, 1930) is in new combination with A. kamitai (Kobayashi, 1934) and A. minjae Hong, 2001 syns. nov. Two newly described Metaphire quelparta sub-spp are M. q. seogwipo and M. q. valhalla these being supported with DNA barcodes despite the nominal taxon not yet being confirmed.
\end{abstract}

Keywords: Annelida, Asian, island biodiversity, native and invasive species, taxonomy

\section{INTRODUCTION}

Jeju-do (제주도 also spelt Cheju-do or Chejoo-do and formerly known to Europeans as Quelpart Isl. or to the Japanese as Saishu-to was historically called Doi amongst other Korean names) is a special autonomous province of $1,849 \mathrm{~km}^{2}$ located $80 \mathrm{~km}$ South of the South Korean mainland. Volcanic origin commenced approximately 2 $\mathrm{Ma}(\mathrm{BP})$ and it was last connected to the Korean Peninsula approximately 10,000 years ago. Its highest mountain Hallasan or Mt Halla, amongst several previous names being known to Europeans as Mt Auckland or to the Japanese as Mt Kanra, is a National Park that supports 1,565 vascular plants and approximately 1,000 identified insects. It is a shield volcano at $1,950 \mathrm{~m}$ in the centre of the island; thus the island's basaltic soils are derived chiefly from lava. Climate is subtropical oceanic and tem- perate supporting a varied flora and fauna that is listed as a UNESCO World Heritage Site for its Volcanos and Larval Tubes (Wikipedia).

The first systematic earthworm survey by Kobayashi (1937) recorded five species, and another five were added by Kobayashi $(1938 ; 1941)$, the types now lost. Thirty years later, Song \& Paik (1970) found six known, three previously unrecorded and one new species. Their total of eleven species then raised the island count to 14 species of earthworms.

Subsequently, Hong \& James (2001a) claimed Amynthas youngtai from Mt Halla, but this is a synonym of $A$. carnosus (Goto \& Hatai, 1899) (see Blakemore, 2012a; 2012f). Also, Amynthas yongshilensis Hong \& James, 2001b: 80 - a probable junior synonym of $A$. tokioensis (Beddard, 1892) - was collected from Mt Halla (=Yongshil?) and was sympatric on Jeju and compared only to their Amynthas alveolatus Hong \& James, 2001b: 81 
from Sangumburi Crater, Jeju that is exactly the same, i.e., synonymous, with A. kanrazanus incretus (Kobayashi, 1937) which is comparable to A. kanrazanus kanrazanus (Kobayashi, 1937: 340, figs. 3, 4) itself also similar to A. tokioensis. Amynthas sangumburi Hong \& Kim, 2002 also from Sangumburi Crater near Mt Halla resembles both A. toriii (Ohfuchi, 1941: 244) from a cave in Oita-ken and $A$. subrotunda (Ishizuka, 2000) both currently in synonymy of $A$. corticis. Therefore, A. sangumburi possibly also belongs to the synonymy of either or joins both of these in A. corticis, at least its validity depends upon deeper research.

Most recently, several exotics and a translocated endemic, Amynthas tralfamadore Blakemore, 2012, were newly reported in Blakemore et al. (2012) and by Blakemore (2012d).

Previous results are compared and consolidated in this taxonomic report following collecting trips by the current author in 2012 to give a current total of more than 40 taxa.

\section{Materials ANd Methods}

Surveys were under the auspices of NIBR with funding from Hanyang University. Taxonomic determinations follow the methodology and classifications in Sims \& Easton (1972) and Blakemore (2010b; 2012a). Abbreviations are: C - circumference, GMs - genital markings, lhs - left hand side and rhs - right hand side. A “?” indicates some uncertainty.

Small tissue samples were taken for mtDNA COI gene barcoding by various Korean Companies with preliminary nucleotide analysis via 'MEGA 5' (wwww.megasoftware. net) and BLAST programs (www.blast.ncbi.nlm.nih.gov/ BLAST.cgi) provided in an Appendix. Those barcode results that are confidently proven will eventually be uploaded to GenBank (www.blast.ncbi.nlm.nih.gov/genbank) and Bold Systems (www.boldsystems.org) or the International Barcode-of-Life project (iBOL www.barcodeoflife. org) with published names.

\section{TAxonomic Results}

Results (arranged by family) are summarized in Table 1.

\section{Enchytraeidae}

Several small, pale, semi-terrestrial "pot-worms" were noted, but not kept, during the surveys and, since they are ecologically ineffectual when compared to megadriles (usually being grouped with the aquatic microdriles) nor are they taxonomically diverse (just 650 named spp from sodden soils worldwide), thus they do not warrant further consideration.

\section{Moniligastridae}

\section{Drawida anchingiana anchingiana Chen, 1933}

Material examined. None. Types not known. Remarks. Drawida gisti anchingiana Chen, 1933: 202 was elevated to specific rank (in part?) by Gates (1935: 3 ) and Kobayashi (1937: 333, fig. 1). It is comparable to Drawida gisti Michaelsen, 1931 as redescribed by Gates (1936: 406-407) that differed from Chen's (1933) version. Both Gates (1935: 2) and Kobayashi (1937: 336; 1938: 35, fig. 2) noted that Chen's description of D. gisti f. typica also differed in some important characters from Michaelsen's original and from his types as later redescribed by Gates (1937). Gates (1935: 3) had earlier noted:

"D. gisti var. anchingiana Chen, 1933, is not adequately described but differs from the [gisti] types as follows: limitation of the penis pouches (?) to the body wall, small size and smooth surface of the prostates, and the presence of the spermathecal atria in vii rather than viii. These differences are important enough to distinguish the worms specifically from $D$. gisti. The specimens may not be sexually mature - vide absence of granulations on the prostates, the empty ovisacs, and the small size of the spermathecal ampullae, as well as the indistinctness of the clitellum."

Kobayashi (1937: 337) concluded that the penial apertures were situated "nearer to b" in D. gisti Michalsen, 1931, but "much nearer to $c$ " in D. anchiangiana and that the spermathecal duct "opens into the broad distal end of a large long sac-like spermathecal atrium" in D. gisti, but "enters into posterior ectal third of the atrium" in D. anchingiana. Kobayashi had as its characteristic feature the spermathecal atrium in segment 7 that was "often" accompanied by an accessory gland (Kobayashi, 1937: 337, fig. 1B). However, it is not clear that what Kobayashi claimed from Jeju was the same as the Chinese taxon due partly to uncertainty of Chen's taxon obtained from "Anching, Anhwei and Pukow, Kiangsu", and due partly to Kobayashi's specimens that, as well as being mostly immature, also tending to vary and perhaps representing a species-complex. Types unknown, this taxon has not been confirmed in China nor Korea - and neither has its sibling species Drawida gisti nanchingiana Chen, 1933: 200. Kobayashi obtained specimens from Jeju town, from Mt Halla and from Seogwipo, some of which probably come close to the following sub-species newly found on Jeju that themselves vary somewhat but which provide objective DNA data for comparison. 
Table 1. Summary of Jeju earthworms survey results

\begin{tabular}{|c|c|c|c|}
\hline No. & Kobayashi $(1937 ; 1938 ; 1941)$ & Song \& Paik (1970) & Recent studies \\
\hline 1 & & & ?Enchytraeus spp. \\
\hline 2 & Drawida anchingiana & & Drawida a. anchingiana \\
\hline 3 & & & Drawida a. halla \\
\hline 4 & & & Drawida a. seogwipo \\
\hline 5 & & & Drawida iисn \\
\hline 6 & Drawida japonica & & Drawida japonica \\
\hline 7 & & & Drawida spp? \\
\hline 8 & & & Ocnerodrilus occidentalis \\
\hline 9 & & & Amynthas arx \\
\hline 10 & & & Amynthas aucklandis \\
\hline 11 & Pheretima carnosa & Pheretima carnosa & Amynthas carnosus \\
\hline 12 & & Pheretima heteropoda & Amynthas corticis corticis \\
\hline 13 & & & Amynthas c. saeseum \\
\hline 14 & & & ?Amynthas gracilis gracilis \\
\hline 15 & & & Amynthas g. insularum \\
\hline 16 & Pheretima hupeiensis & Pheretima hupeiensis & Amynthas hupeiensis \\
\hline 17 & Pheretima kanrazana kanrazana & Pheretima kanrazana & Amynthas kanrazanus kanrazanus \\
\hline 18 & Pheretima kanrazana increta & Pheretima kanrazana & Amynthas $k$. incretus (inc. alveolatus) \\
\hline 19 & Pheretima masatakae & Pheretima masatakae & Amynthas masatakae \\
\hline 20 & & & Amynthas micronarius \\
\hline 21 & & Pheretima phaselus var. kamitai & Amynthas phaselus maculosus (incl. kamitai and minjae) \\
\hline 22 & & & ?Amynthas p.phaselus \\
\hline 23 & & & ?Amynthas sangumburi \\
\hline 24 & & Pheretima seungpanensis & Amynthas seungpanensis \\
\hline 25 & & & Amynthas simplex \\
\hline 26 & & & Amynthas tokioensis (incl. yongshilensis) \\
\hline 27 & & & Amynthas tralfamadore \\
\hline 28 & & Pheretima agrestis & Metaphire agrestis \\
\hline 29 & & & Metaphire californica \\
\hline 30 & & & Metaphire haenyeo \\
\hline 31 & & & ?Metaphire hilgendorfi \\
\hline 32 & Pheretima quelparta & Pheretima quelparta & Metaphire quelparta quelparta \\
\hline 33 & & & Metaphire q. seogwipo \\
\hline 34 & & & Metaphire q. valhalla \\
\hline 35 & & Pheretima soulensis & Metaphire soulensis \\
\hline 36 & & & Pontodrilus litoralis \\
\hline 37 & & & Aporrectodea caliginosa/tuberculata \\
\hline 38 & $\begin{array}{l}\text { Allolobophora caliginosa } \\
\text { trapezoides? }\end{array}$ & Allolobophora cal. trapezoides & Aporrectodea trapezoides \\
\hline 39 & & & Bimastos parvus \\
\hline 40 & & & ?Dendrobaena sp \\
\hline 41 & & & ?Eisenia andrei \\
\hline 42 & & & Eisenia fetida \\
\hline 43 & Allolobophora japonica typica & & Eisenia japonica \\
\hline Total & 10 & 12 & 43 \\
\hline
\end{tabular}

\section{Drawida anchingiana halla sp. nov.}

\section{[Fig. 1]}

Material examined. IV0000251105 Holotype, figured and dissected with tissue samples taken for DNA analysis [WO46 (cf. WO45), w24, w43]. Collected $12^{\text {th }}$ June 2012 by RJB from Hyonyungsa Temple, Mt Halla $\left(33^{\circ}\right.$ $21^{\prime} 42^{\prime \prime} \mathrm{N} 126^{\circ} 31^{\prime} 45^{\prime \prime} \mathrm{E}$ ); preservation in $80 \% \mathrm{EtOH}$.

Note. Superficially similar specimens from Mt Halla looked possibly conspecific but also resemble $D$. koreana Kobayashi, 1938 are noted as "Drawida sp.2" below.
Etymology. From location, noun in apposition.

Description. Length $60 \mathrm{~mm}$. Segments 128. Body stout, flattened posteriorly. Colour dark brownish in anterior and bluish in posterior. Prolobous, ventrally cleft. No dorsal pores. Clitellum not developed. Nephropores in $\mathrm{cd}$ lines. Spermathecal pores in 7/8 median of c lines. Male pores on penes protruding slightly from wide slits centred median of $\mathrm{c}$ lines in 10/11. Female pores in $\mathrm{b}$ lines in $11 / 12$. Genital markings are widely paired presetally median of c lines in 8 and 10 (circular 'GM glands' correspond internally, DNA sample from 8lhs that appeared 

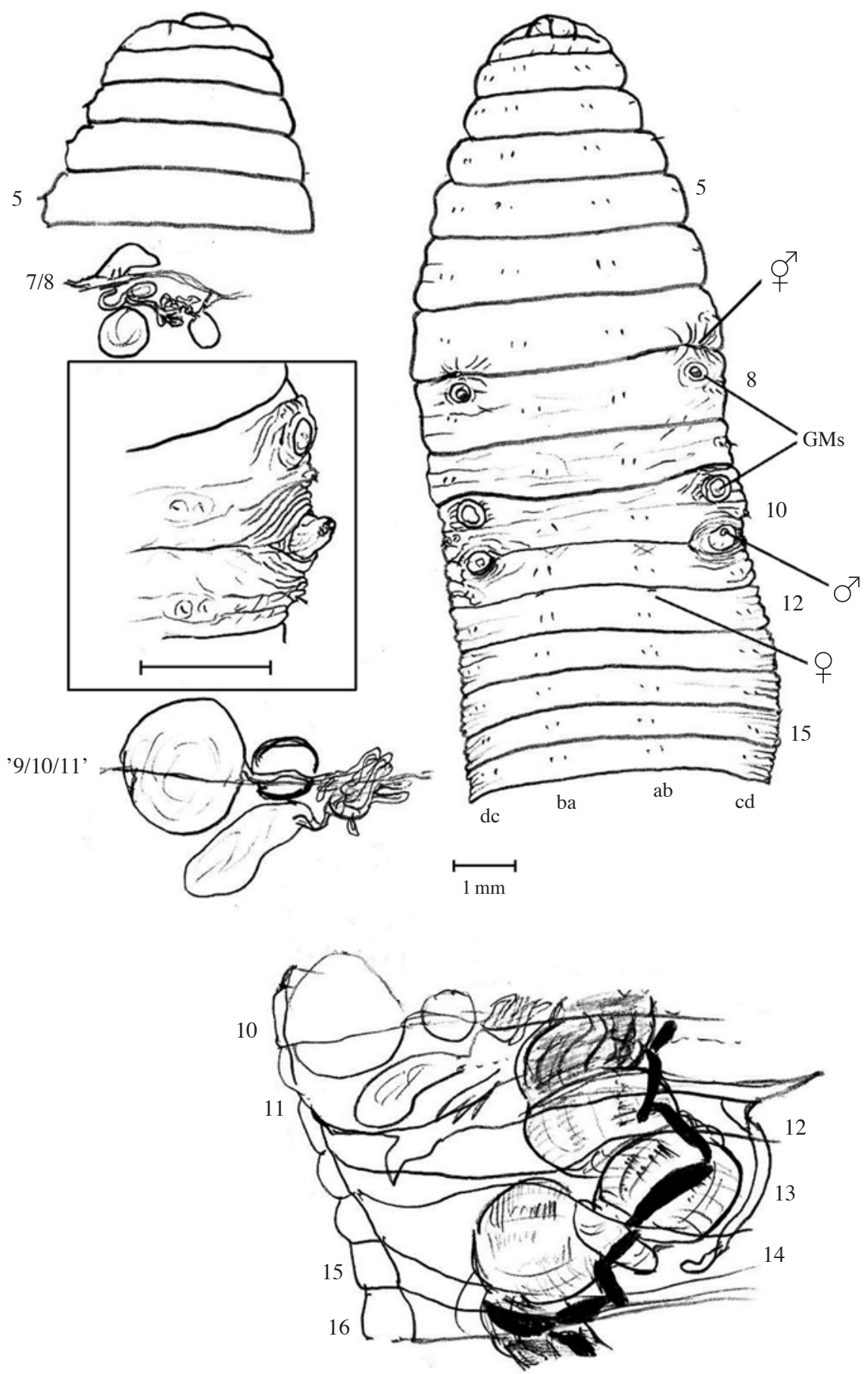

Fig. 1. Drawida anchingiana halla sp. nov. holotype showing ventral aspect, prostomium; gizzards and reproductive organs with [boxed] enlargment of male pore.

to contain mucus and a nematode - w43).

Internally, septa 5/6-8/9 thick; others thin and displaced; 10/11 supports large testis sac with coiled vas deferens to prostate near exit. Dorsal blood vessel single and hearts in 6-9. Spermathecae atria present anteriorly from $7 / 8$ with long coiled duct to ampulla attached to $7 / 8$ pos- teriorly in 8. Ovisacs small (empty) from septum 12/13; $11 / 12$ doubled to form an ovarian chamber. Nephridial bladders elongate. Gizzards in 12-14.

Remarks. Drawida gisti Michaelsen, 1931 that occurs on the mainland (Kobayashi, 1938: 35) has male pores nearer to $\mathrm{b}$ lines and gizzards in 12-14, whereas D. anchingi- 
ana has male pores nearer to c lines and gizzards described by Kobayashi (1937: 335) as three in "XII-XVI or $X I-X V I$ " with markings variable in some of 8,10 and 11 and either presetal or postsetal; plus a distinctive accessory atrial gland in segment 7. Drawida a. halla sub-sp. nov. has male pores near c lines, gizzards in 12-14 plus widely paired and presetal genital markings in 8 and 10 that, in combination with DNA data in Appendix identify this species.

\section{Drawida anchingiana seogwipo subsp. nov.} [Fig. 2]

Material examined. IV0000261226 Holotype (H) figured and dissected with small tissue samples taken for DNA analysis (WO69, w44); IV0000261228 Paratype (P) providing DNA sample (WO71). Collected $9^{\text {th }}$ Sept. 2012 by RJB during short walk from Botanic Gardens to IUCN meeting at Seogwipo $\left(33^{\circ} 15^{\prime} 10^{\prime \prime} \mathrm{N} 126^{\circ} 33^{\prime} 40^{\prime \prime} \mathrm{E}\right)$; preservation in $80 \% \mathrm{EtOH}$.

Note. Three complete specimens and two fragments, with the same collection data but none dissected, are possibly the same taxon (see "Drawida sp.1" below).

Etymology. After the type-locality, noun in apposition.

Description. Length 55-58 mm (H missing tip of tail, $\mathrm{P}$ complete). Colour bluish in posterior of body, with faintly dark mid-dorsal line. Prolobous, ventrally cleft. No dorsal pores. Clitellum not developed. Nephropores clearly in setal d lines or above in anterior. Spermathecal pores in $7 / 8$ in cd lines. Male pores on penes protruding slightly from wide slits centred on c lines in 10/11. Female pores in $b$ lines in 11/12. Genital markings paired in 8 offset medially, and lhs analogues in 9, 10 and 11 in $\mathrm{H}$; $\mathrm{P} 1$ has them on $10 \& 11$ only (circular 'GM glands' correspond internally - on the off-chance they are parasitic artefacts those from 8rhs and 10lhs from $\mathrm{H}$ sampled for DNA w44, results in Appendix).

Internally, septa 5/6-8/9 thick; 9/10 and 10/11 are conjoined and displaced to mid-10 supporting yellow testis sac with coiled vas deferens to prostate that attaches to male organ. Dorsal blood vessel single and hearts in 6-9. Spermathecae atria present anteriorly from $7 / 8$ with long coiled duct to ampulla attached to $7 / 8$ posteriorly in 8 . Ovisacs (empty) present from septum 12/13 that along with 11/12 forms an ovarian chamber ventrally. Nephridial bladders elongate. Gizzards muscular in 13-15.

Remarks. Differences in the current type of $D$. a. seogwipo from the nominal taxon are gizzards in 13-15 and lack of an atrial gland which may be its distinguishing characters, here complemented with the DNA barcode data including that of the paratype (WO71) that was not so extensively studied but differs only slightly. Morphological similarity of $D$. anchingiana seogwipo to $D$. $a$. halla sub-sp nov. described above, extends to atrium in
7 and lack of accessory glands there, but is contraindicated by gizzards in 13-15 rather than 12-14. Slight DNA barcode indications support its separation (w24 vs. WO69 in Appendix). However, should D. a. anchingiana proper be confirmed from China (or Jeju!) then morphological and genetic comparison may allow clarification of the full species relationship.

Both new sub-species may indicate a variable species complex, possibly with parts similar to what Kobayashi found. Mutual inclusion or separation depends on relocating fully mature material on Jeju that correspond to Kobayashi's description, plus further studies of types of the whole species-complex in China.

\section{Drawida iucn sp. nov.} [Fig. 3]

Material examined. IV0000261225 Holotype, figured (Fig. 3) and dissected with small tissue sample taken for DNA analysis (WO68). Collected $9^{\text {th }}$ Sept. 2012 by RJB from under rock on short walk from Botanic Gardens to IUCN meeting at Seogwipo ( $\left.33^{\circ} 15^{\prime} 10^{\prime \prime} \mathrm{N} 126^{\circ} 33^{\prime} 40^{\prime \prime} \mathrm{E}\right)$; preservation was in $80 \% \mathrm{EtOH}$.

Etymology. From acronym for International Union of Conservation Networks who hosted the World Conservation Congress at Jeju and are concerned with promotion of species diversity. This does not imply support nor funding for the survey and its results from IUCN. Pronounciation is "eye-you-see-en", a non-arbitrary combination of letters used as a word formed from an acronyms as permitted under ICZN recommendations (cf. Eisenia anzac Blakemore, 2011 from Japan).

Description. Length $73 \mathrm{~mm}$. Segments 127 , possibly the last 12 regenerated. Colour pale grey unpigmented with dark mid-dorsal line from 11. Prolobous. No dorsal pores. Clitellum not developed. Nephropores in c lines or above. Spermathecal pores in $7 / 8$ in c lines. Male pores on blunt penes protruding slightly from wide slits centred median of cd lines in 10/11. Female pores in b lines in 11/12. Genital markings single, mid-ventrally offset in 8 and 11.

Internally, septa 5/6-8/9 thick; $9 / 10$ and 10/11 are conjoined and displaced to mid-10 supporting yellow testis sac with coiled vas deferens to prostate that attaches to male organ. Dorsal blood vessel single and hearts in 6-9. Spermathecae atria present anteriorly from $7 / 8$ with long coiled duct to ampulla attached to $7 / 8$ posteriorly in 8 . Ovisacs (empty) present from septum 12/13 that along with $11 / 12$ forms an ovarian chamber ventrally. Nephridial bladders elongate. Gizzards in 12-15 with muscular modification in 12 reduced.

Remarks. Drawida iucn agrees morphologically with $D$. anchingiana seogwipo on almost all points, except for lack of blue colour, GM details and extension of gizzards into 12. Moreover, the DNA barcode data in Appendix 


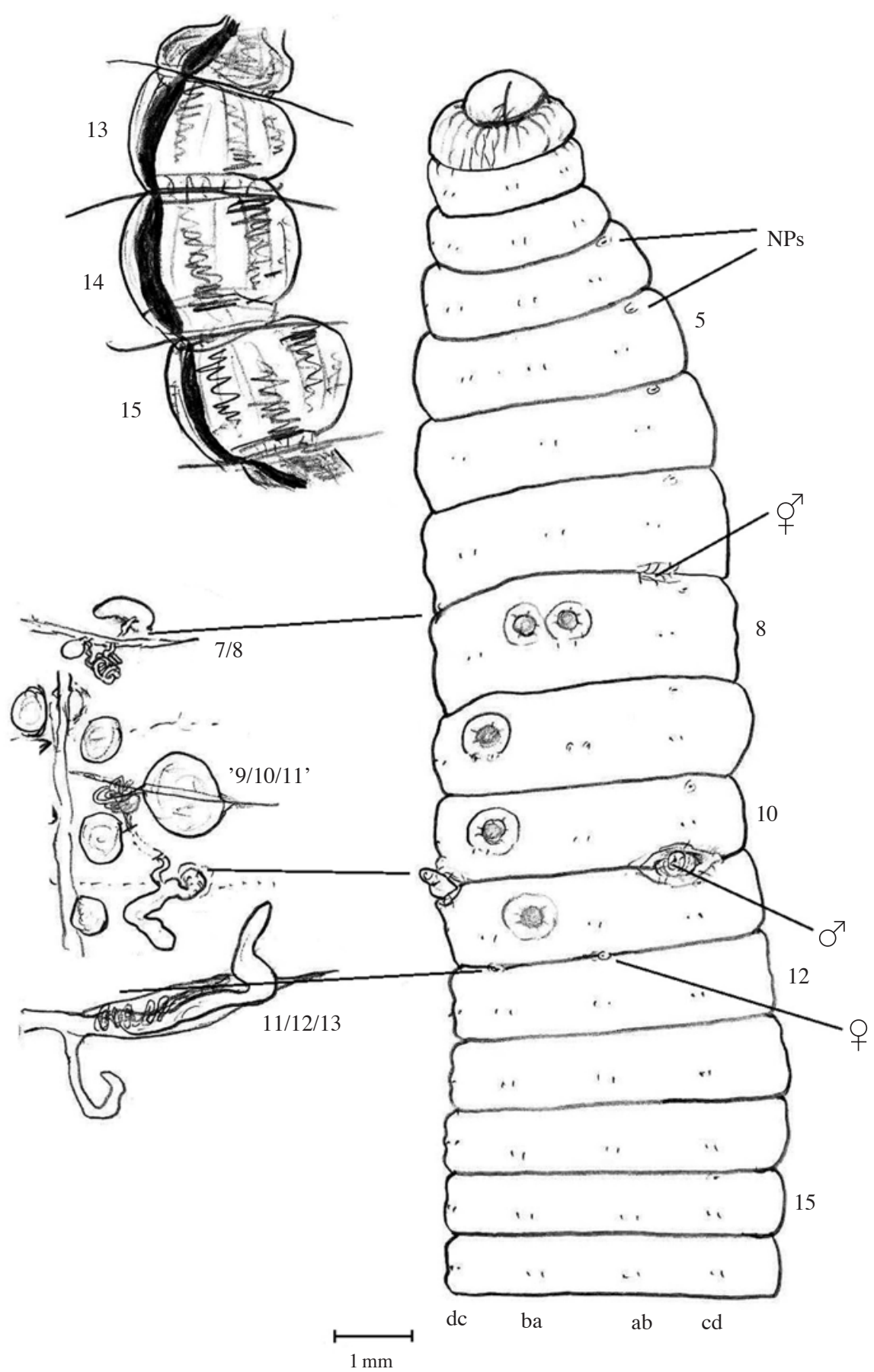

Fig. 2. Drawida anchingiana seogwipo sub-sp. nov. holotype showing ventral aspect; gizzards and reproductive organs.

(WO68 vs. WO69) shows that there is some difference $(3 \%)$ that is here considered sufficient to indicate speciation. D. iucn barcode also differs (4\%) from D. a. halla (WO68 vs. w24), supporting their mutual separation.

\section{Drawida japonica japonica (Michaelsen, 1892)}

Material Examined. Hamburg Museum and Berlin syn- types were inspected by the author as reported in Blakemore \& Kupriyanova (2010: 8) wherein two synonyms were listed: Drawida grahami Gates, 1935 and Drawida propatula Gates, 1935.

Remarks. Reported from Japan, Korea, China, Taiwan, Southeast Asia and India. Not confirmed on Jeju since Kobayashi (1938: 95) (cf. "Drawida sp 2" below); it is fully redescribed on types in Blakemore \& Kupriyanova 


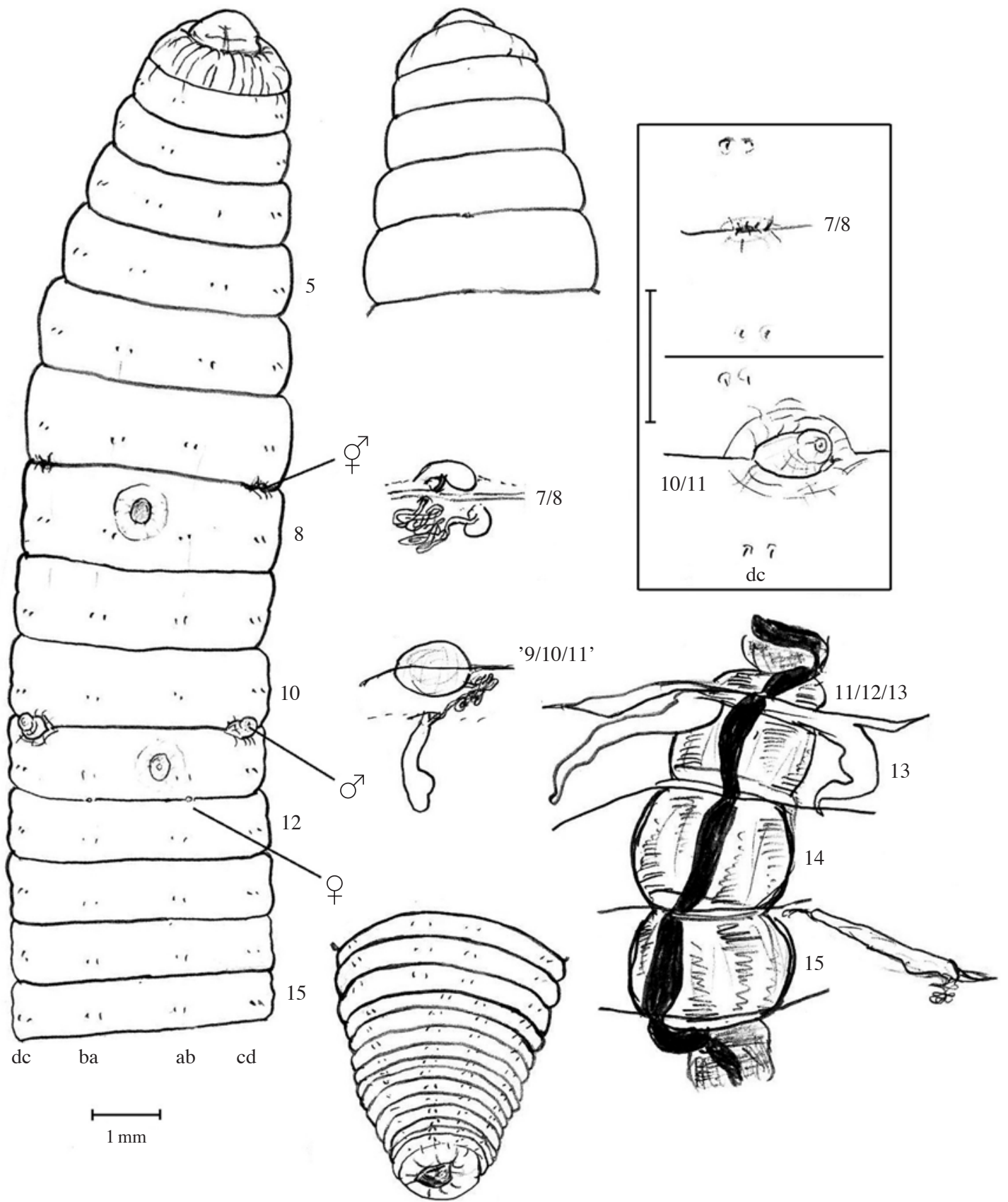

Fig. 3. Drawida iucn sp. nov. holotype showing ventral aspect, prostomium and pygidium; gizzards and reproductive organs with [boxed] enlargement of spermathecal and male pore.

(2010).

\section{Drawida sp. 1 (cf. D. a. seogwipo)}

Material examined. IV0000261227 posterior amputee specimen providing DNA (WO70), collected $9^{\text {th }}$ Sept. 2012 by RJB during a short walk from Botanic Gardens to IUCN meeting at Seogwipo, plus IV0000261229 of two complete and two fragments, with the same collec- tion data. None yet dissected.

Remarks. Sympatric and probably closest to D. a. seogwipo (see Appendix and Fig. 4).

\section{Drawida sp. 2 (cf. D. koreana)}

Material examined. IV0000249942 mature specimen undissected, from under bridge on Rt. 516, Mt Halla, $12^{\text {th }}$ June, 2012 collected by RJB and NIBR team, providing 
DNA sample (WO44 that was mixed in the lab, resampled as w17). IV0000249943, two similar semi-matures undissected, possibly the same taxon, from the same location.

Remarks. Specimens a blue colour considered to be Drawida $\mathrm{cf}$. koreana are unidentified at this time and await thorough analysis of D. koreana from the mainland. Summary of its DNA data thus far are provided in the Appendix.

Jeju results for all Drawida spp. are summarized in Table 2 and Fig. 4 (a Maximum Likelihood Tree automatically generated by MEGA 5 program).

\section{Ocnerodrilidae}

\section{Ocnerodrilus occidentalis occidentalis Eisen, 1878}

Material examined. IV0000245077 collected from Jeju Island by the author (15.II.2012) as reported in Blakemore et al. (2012, fig. 1) providing DNA sample (WM17 in Appendix).

Remarks. Pan-tropical distribution by introduction; the original home is thought to be in Central/South America; it is fully described by Blakemore (2010b). Part of a species-complex (Gates, 1972), previously noted without details from North Korea by Kobayashi (1941: 263, tab. 1) a record that appears to have been overlooked subsequently due to the old-style kanji used.

\section{Megascolecidae}

\section{Amynthas arx sp. nov.}

\section{[Fig. 5]}

Material examined. IV0000246444 holotype (H) submature specimen, sketched and dissected providing DNA sample (WM11 resampled as w4), collected from side of Haenyeos' beach at base of Seongsan Ilchulbong or "Sunrise Peak", Jeju $15^{\text {th }}$ Feb., 2012 by RJB.

Etymology. Latin noun 'arx' for 'citadel or fortress' after the location's colloquial name of 'The Floating Fortress'. Description. Length $92 \mathrm{~mm}$, segments 102. Colour in life a deep red-brown, more a chocolate-like colour in alcohol with a paler ventrum and darker mid-dorsal line. Prostomium epilobous. First dorsal pore in 12/13. Setae ca. 6070 per segment. Clitellum slightly darker in $14-16$. Spermathecal pores lateral in 6/7/8/91hs and 5/6/7/8/9rhs. Female pore obscure. Male pores widely paired superficial on raised white pads but within curved, longitudinal slits on 18 in shape of deeply incised "("and")" braces, with 35 much crowded small penial setae intervening, most concentrated nearer to the slits. Genital markings

Table 2. Contingencies of Jeju Drawida spp compared to D. koreana Kobayashi, 1938*

\begin{tabular}{|c|c|c|c|c|}
\hline Taxon & Male pores & Gizzards & GMs & Atrium (gland) \\
\hline D. a. anchingiana & Nearer c & Three (in 11,12-16?) & $(8,10,11 ?)$ & $7(Y)$ \\
\hline D. a halla & Nearer c & Three (12-14) & 8 and 10 wide & $7(\mathrm{~N})$ \\
\hline D. a. seogwipo & Nearer c & Three (13-15) & 8-11 variable & $7(\mathrm{~N})$ \\
\hline D. iucn & Nearer c & Four $(1 / 212-15)$ & 8 and $11 \mathrm{mid}$ & $7(\mathrm{~N})$ \\
\hline D.j. japonica & Near b & Two/three $(12-13,14)$ & $7-13$ or absent & $8(\mathrm{~N})$ \\
\hline D. koreana* & Near b & Two/three $(12-13,14)$ & 7-13 variable- & $8(\mathrm{~N})$ \\
\hline
\end{tabular}

*D. koreana is a deep blue colour, which helps to distinguish it from pink/grey $D$. japonica.

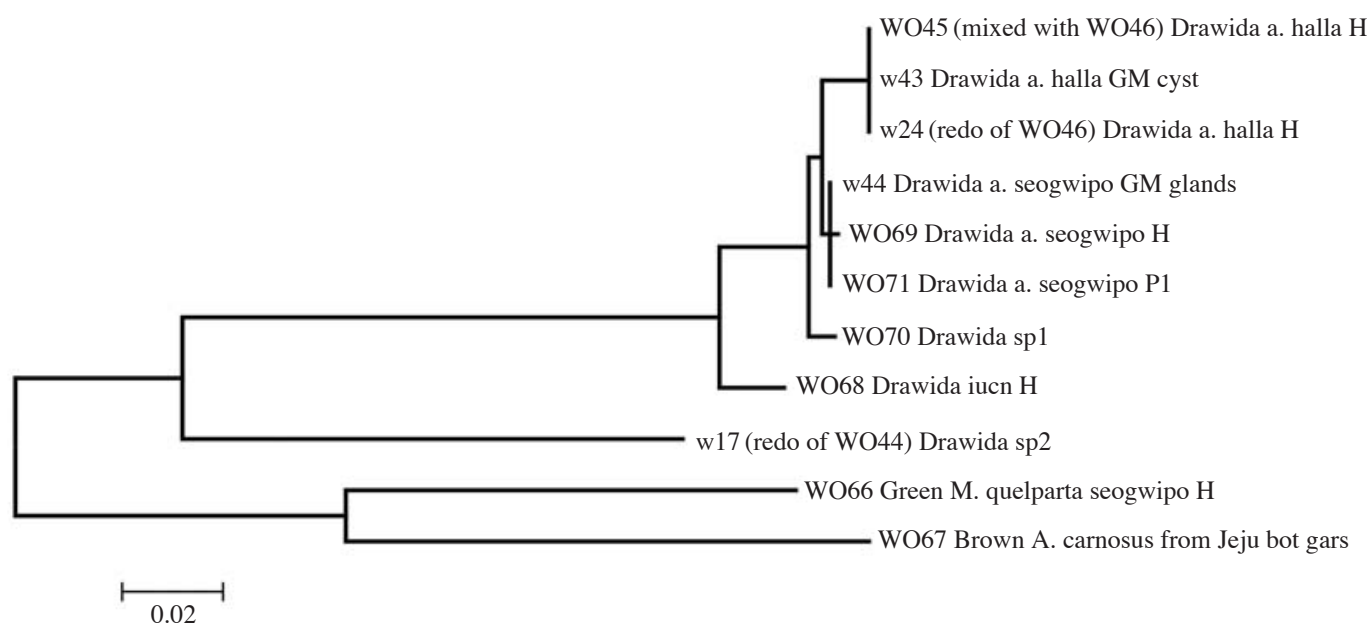

Fig. 4. Phylogram of DNA barcodes for Jeju Drawida spp. compared to megascolecids. 


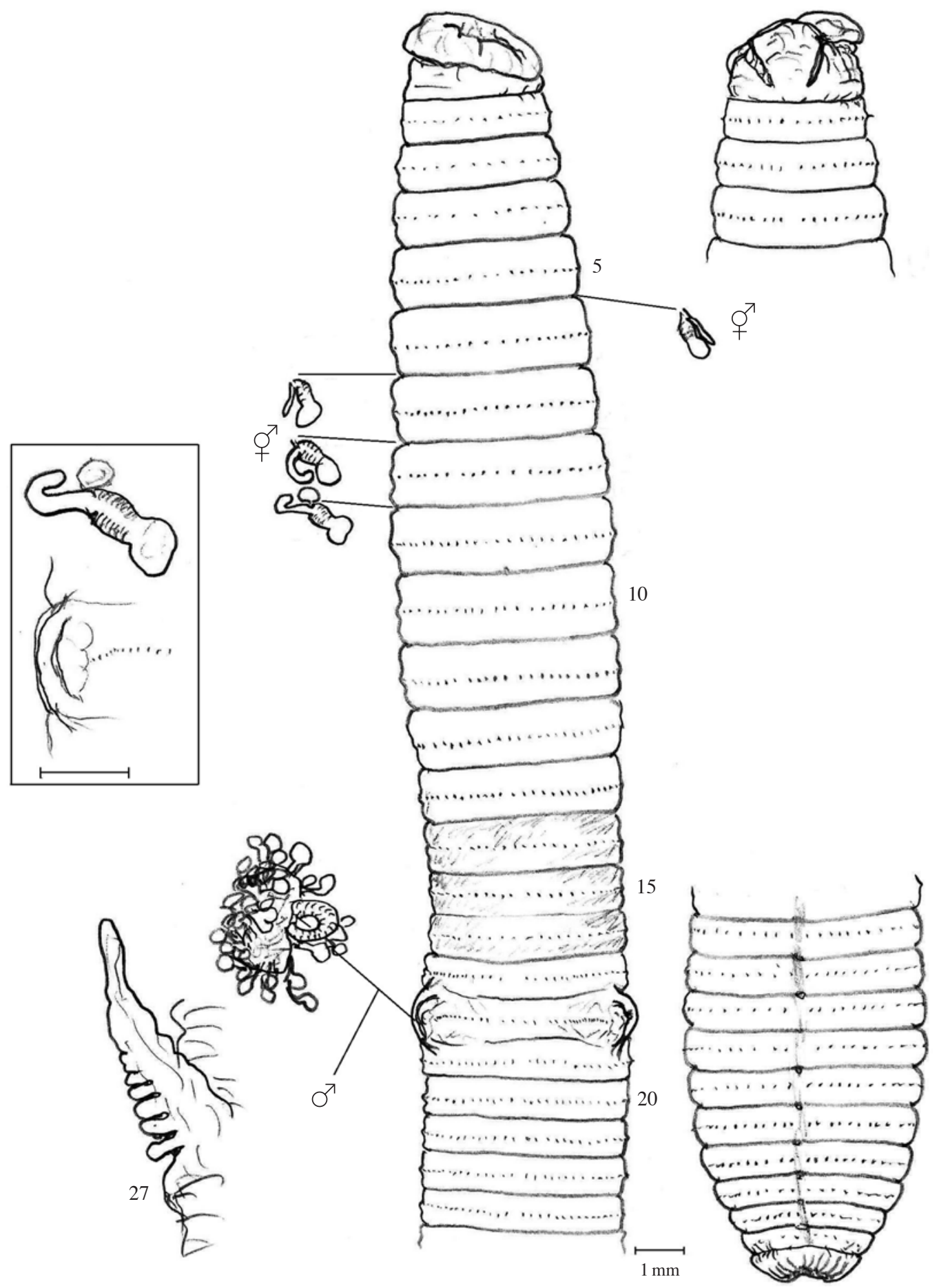

Fig. 5. Amynthas arx sp. nov. holotype showing ventral aspect; prostomium and pygidium, spermathecae, prostate and caecum in situ with [boxed] enlargement of spermatheca in 9 and of male pore 18lhs].

not found although glands present internally in 9rhs and 9lhs.

Internally, the pharyngeal mass fills segment 4 . Septa 5/6/7/8 weak, 8/9/10 absent around muscular gizzard, 10 /11-13/14 slightly thicker. Dorsal blood vessel single, hearts in 10-13. Spermathecae in 6-9lhs \& 7-9rhs, small with thick muscular duct and roundish ampulla with curved diverticulum (non iridescent). Glands occur near spermathecae in 9. Holandric but testis small and non iridescent; seminal vesicles anteriorly in $11 \& 12$. Prostates 
racemose, heavily parasitized in 18 . Ovaries flat in 13 with no ovisacs seen in 14. Oesophagus dilated in 11-13, intestinal origin in 15 . Intestinal caeca from 27, hemi-pinnate ventrally. Low but wide typhlosole from ca. 25. Gut contains soil and grits. Gregarine parasites around prostates as noted but no other parasites found.

Remarks. Specimen distinct with its red colouration, but possibly not fully mature. Its $\mathrm{C}$-shaped seminal grooves, crowded setae and incised caeca appear unique. The male pores are construed as superficial rather than eversible as in Metaphire spp. and, assuming spermathecae in 5/6/7/8/9, the species would comply with an Amynthas corticis species-group of Sims \& Easton (1972). Possibly a 'species-in-transition', losing its anterior spermathecae and approaching Metaphire with quasi-non-superficial male pores. By extension, in Metaphire it would comply with an $M$. ignobilis species-group and the taxon it would resembles most on Jeju probably being Metaphire quelparta (Kobayashi, 1937) from which it differs principally in the visibility of primary male pores, the incipient retention of spermatheca in 6lhs, colouration plus other lesser characters. The penial setae, uniquely characteristic, possibly provide traction during copulation and compensate for lack of genital markings. DNA data (w4 in Appendix) confirm its identity and shows closest match with A. aucklandis (w21). However, such DNA data cannot be used to determine whether it is correctly placed in Amynthas, the default genus for pheretimoids (see Blakemore, 2012e).

\section{Amynthas aucklandis sp. nov.}

[Fig. 6]

Material examined. Holotype (H) IV0000251100 mature, from Mt Halla under bridge on Rt $51612^{\text {th }}$ June, 2012; sketched and dissected providing DNA samples (WO41 resampled as w21).

Etymology. From Mt Auckland (Latin genitive) the European's early name for Mt Halla.

Description. Length 120; segments 98. Colour dark brown on dorsum and anterior. Clitellum buff 14-16. Epilobous. First dorsal pore in $12 / 13$. Setae number about 64 per segment. Spermathecae in wide slits in 6/7/8/9 about 0.3 $\mathrm{C}$ apart. Female pores single on 14. Male pores deep in lateral C-shaped clefts (seminal grooves), the male porophore body appears retractable as a flat pad with five or six setae above a circular disc. Genital markings, in addition to those on male pads, also as small discs lateral of spermathecal pores, plus one pair below 7/8 and another postsetal pair ventrally on 8 .

Internally, nephridial forests fill 5 and 6. Septa are all thin, 8/9/10 are absent in position of muscular gizzard. Hearts in 10-13. Spermathecae have a large saccular ampula on muscular duct with long diverticulum inseminat- ed for most of its length. Small glands associated with each spermatheca and in place of GMs. Holandric, with large seminal vesicles in $11 \& 12$. No vesicles in 13 . Prostates on thick duct to infolding of male pores. Ovaries free in 13 , no ovisacs in 14 . Oesophagus slightly dilated in 13 only, intestine from 15, caeca broad based from 27 and deeply incised ventrally. Parasites not noted.

Remarks. As with $A$. arx, the male pores are construed as within $\mathrm{C}$-shaped seminal grooves rather than non-superficial and eversible as in Metaphire. The current species differs from A. arx, M. quelparta and other similar species described herein in the shape of its spermathecae and distinctive male field. DNA data are provided for confirmation (Appendix).

\section{Amynthas carnosus (Goto and Hatai, 1899)}

Material examined. Tokyo Museum neotype (An435) designated and described by Blakemore (2012a). IV000 0261224 from Seogwipo found during walk from Botanic Gardens, 9 ${ }^{\text {th }}$ Sept. 2012 collected by RJB from under rocks beside road, providing DNA sample (WO67). Specimen undissected but is superficially similar to A.carnosus. Remarks. Chinese/Japanese/Korean Amynthas carnosus (Goto \& Hatai, 1899) recently redescribed on the Tokyo museum neotype (Tokyo NSMT An435) by Blakemore (2012a; 2012f) where an annotated synonymy included: kyamikia Kobayashi, 1934, monstrifera Kobayashi, 1936, sangyeoli, youngtai, kimhaeiensis, sinsiensis and baemsagolensis - all Korean names by Hong \& James, 2001, and Taiwanese monsoonus James et al., 2005. However, Chinese A. pingi (Stephenson, 1925) (syn. fornicata Gates, 1935) was maintained separately, at least for the present, until its comprehensive review, possibly extending to DNA barcode differentiation. Distribution is Japan (Goto \& Hatai, 1899; Easton, 1981), Korea and Quelpart Island (Kobayashi, 1936; 1938: 161). Hong \& James (2001a) claimed their Amynthas youngtai from Mt Halla, but this is a clear synonym of A. carnosus (Goto \& Hatai, 1899) after Blakemore (2012a). In addition to these locations, A. carnosus is known from China (Jiangsu, Zhejiang, Anhui, Shandong, Hong Kong, Sichuan, Beijing) and possibly Vietnam (as $P$. pingi). Recent Chinese claims from islands of Hainan as Amynthas fuscus Qiu \& Sun, 2012 and off from Taiwan, as Amynthas taiwumontis Shen et al., 2013 syn. nov. that agree with Kobayashi (1936: fig. 1 types I or II and fig. 2 type I) - see Blakemore (2012a). Both A. fuscus and A. taiwumontis were compared to Amynthas fornicatus (Gates, 1935) which is surprising as it is currently held in synonymy of Chinese A. pingi (Stephenson, 1925), itself usually in synonymy of A. carnosus. Senior synonyms were further ignored as A. fuscus was compared to A. homosetus (Shen, 1938) and the $A$. sangumburi synonyms of $A$. corticis; while $A$. 

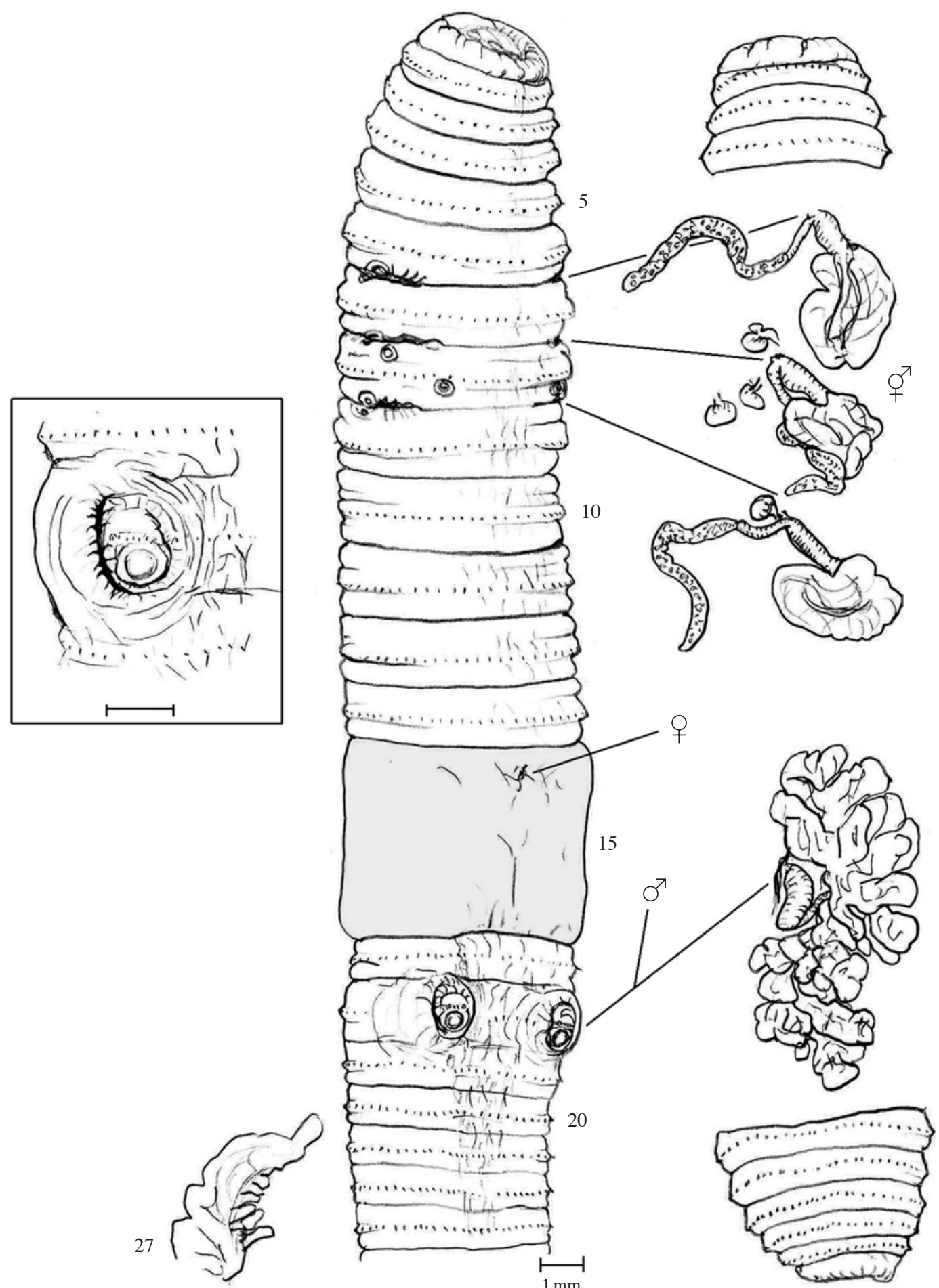

Fig. 6. Amynthas aucklandis sp. nov. holotype showing ventral aspect; prostomium and pygidium, spermathecae, prostate and caecum in situ with [boxed] enlargement of male pore 18lhs].

taiwumontis was also compared to A. marenzelleri (Cognetti, 1906) from Yokohama, a long established synonyn of A. corticis (Kinberg, 1867) despite its recent redescription by Blakemore (2012b: 114). Despite their claim, barcode data for "A. taiwumontis" could be found neither on GenBank nor on iBOLD site of the CCDB (www.ccbd. ca Feb., 2013).

These authors are invited to confirm their taxa, to cor- 
rect their mistakes and to understand that no Asian country is in isolation as the species are often mutually shared. Moreover, ICZN requires consistency across names and treatments regardless of where specimens occur.

\section{Amynthas corticis corticis (Kinberg, 1867)}

Material examined. Five mature specimens from Hyomyungsa Temple, Mt Halla collected $12^{\text {th }}$ June, 2012, one IV0000250394 providing DNA samples (WO47 redone as w25), the remainder labelled IV0000250395. [IV000 0250399 a sub-adult with same collection data that provided DNA (WO50)]. IV0000251208 four mature specimens plus two immatures also identified from Cheonjiyeon Falls, Seogwipo, collected $13^{\text {th }}$ June, 2012.

Note. DNA sample WO56 for "A. agrestis" was mixed in the lab. possibly with WO47 since it showed $100 \%$ megaBLAST agreement with A. corticis.

Remarks. It is one of the most widely cosmopolitan of Megascolecidae, being well-known from China, Japan and Korea including Jeju. Part of an Amynthas corticis species-complex sensu Blakemore (2010b), its many synonyms include Perichaeta heteropoda Goto \& Hatai, 1898: 69 from Japan that, although still quoted as a valid taxon from Korea (e.g. Hong and Kim, 2007; 2009; Kim et al., 2009), has long been held in synonymy of A. corticis and moreover, there are twenty synonyms with priority over heteropoda as fully described in Blakemore (2010b). As already noted, Amynthas sangumburi Hong \& Kim, 2002 is a probable new synonym of A. corticis, here provisionally retained pending further research by its authors.

\section{Amynthas corticis saeseum sub-sp. nov. [Fig. 7]}

Material examined. Holotype IV0000251310, H (mature figured and dissected providing DNA sample (WO57 resampled as w33); Paratypes IV0000251311, P1-3 (three matures, one posterior amputee dissected), $\mathrm{P} 4$ (immature inspected); all from Saeseum Island, Seogwipo, $\left(33^{\circ} 15^{\prime}\right.$ $10^{\prime \prime} \mathrm{N} 126^{\circ} 33^{\prime} 40^{\prime \prime} \mathrm{E}$ ), collected RJB $13^{\text {th }}$ June, 2012 from under rocks beside pathway.

Description. Length $100 \mathrm{~mm}(\mathrm{H})$. Colour brownish with darker dorsum, clitellum buff on 14-16. Epilobous. First dorsal pore in 12/13. Spermathecal pores in 5/6/7/8/9, ca. $0.3 \mathrm{C}$ apart. Female pore single on 14. Male pores absent, replaced by superfical GMs on 18 with ca. 14 setae intervening. Other genital markings variable: paired or analogue discs posteriomedian to spermatheal pores in 7,8-9, each with stalked glands corresponding internally, some of those in P1 with multiple stalked glands numbering up to three per GM in some cases.

Internally, nephridial forests cover septa in 5 and 6.
Spermathecae with wide muscular duct and slightly dilated ampulla with terminal bulb consistently constricted or stalked; diverticulum stunted or with shorter and muscular stalk, the bulb absent or rounded and non-iridescent. Septa 8/9/10 absent around gizzard. Hearts in 10-13. Testis in $10 \& 11$ (non iridescent), seminal vesicles in $11 \&$ 12. Prostates wholly absent, replaced by small gland, indicative of GM on 18 where male pores would be expected. Oesophagus dilated in 11,12-13. Intestine from 15 , caeca simple from 27, typhlosole not noted. Parasites not found.

Remarks. Superficially complying with the nominal taxon, Amynthas corticis saeseum is possibly a 'proto-species' distinguished principally by its consistent bulbous augmentation to the spermathecae on either side. Although only parthenogenetic specimens were found indicating a clonal lineage that is only potentially interbreeding (and intrabreeding), here considered equivalent to an allopatric sub-species. Recent phenotypic divergence supported by its DNA barcode complying with some parts of the $A$. corticis species-complex (WO57 redone as w33 in Appendix) yet separable by about $3 \%$ from "A. corticis" specimens identified on Jeju (e.g. w25 in Appendix) and on the Korean mainland (w59) by the author (Blakemore in prep.). The only other species found on the island was Metaphire californica (IV0000250461 providing DNA WO58 (resampled as w34).

\section{Amynthas gracilis gracilis (Kinberg, 1867) [Fig. 8A]}

Material examined. IV000251208 from Cheonji-yeon Falls, $13^{\text {th }}$ June, 2012. Mature specimen sketched and dissected providing DNA sample (w63).

Description. (Jeju specimen in summary). Genital marking discs median to male pores, postsetally with small stalked glands corresponding internally. Spermathecal pores in $5 / 6 / 7 / 8$, first dorsal pore in $11 / 12$. Septa $7 / 8$ present to base of gizzard, 8/9 absent. Spermathecae in 6-8, with saccular ampulla on muscular duct with thin diverticula stalk having small, spherical bulb. Holandric. Prostates glands well developed in 18. Intestinal caeca incised ventrally. Typhlosole absent.

Remarks. The current specimen complies morphologically with current concepts of "A. gracilis" (e.g., by Gates, 1972; Sims \& Gerard, 1999; Blakemore, 2010b), another cosmopolitan species from tropical and warm temperate localities. Blakemore (2010b) noted that Amynthas bouchei, A. omodeoi and A. edwardsi all by Zhao \& Qiu in Zhao et al., 2009 from Hainan, China in the same " $h a$ wayanus species-group", are possible syns. nov. of $A$. gracilis. Sims \& Easton $(1972:$ 176, 214) remarked that gracilis was the type-species of the monotypic genus Nitocris Kinberg, 1867, a pre-occupied name, and made it 


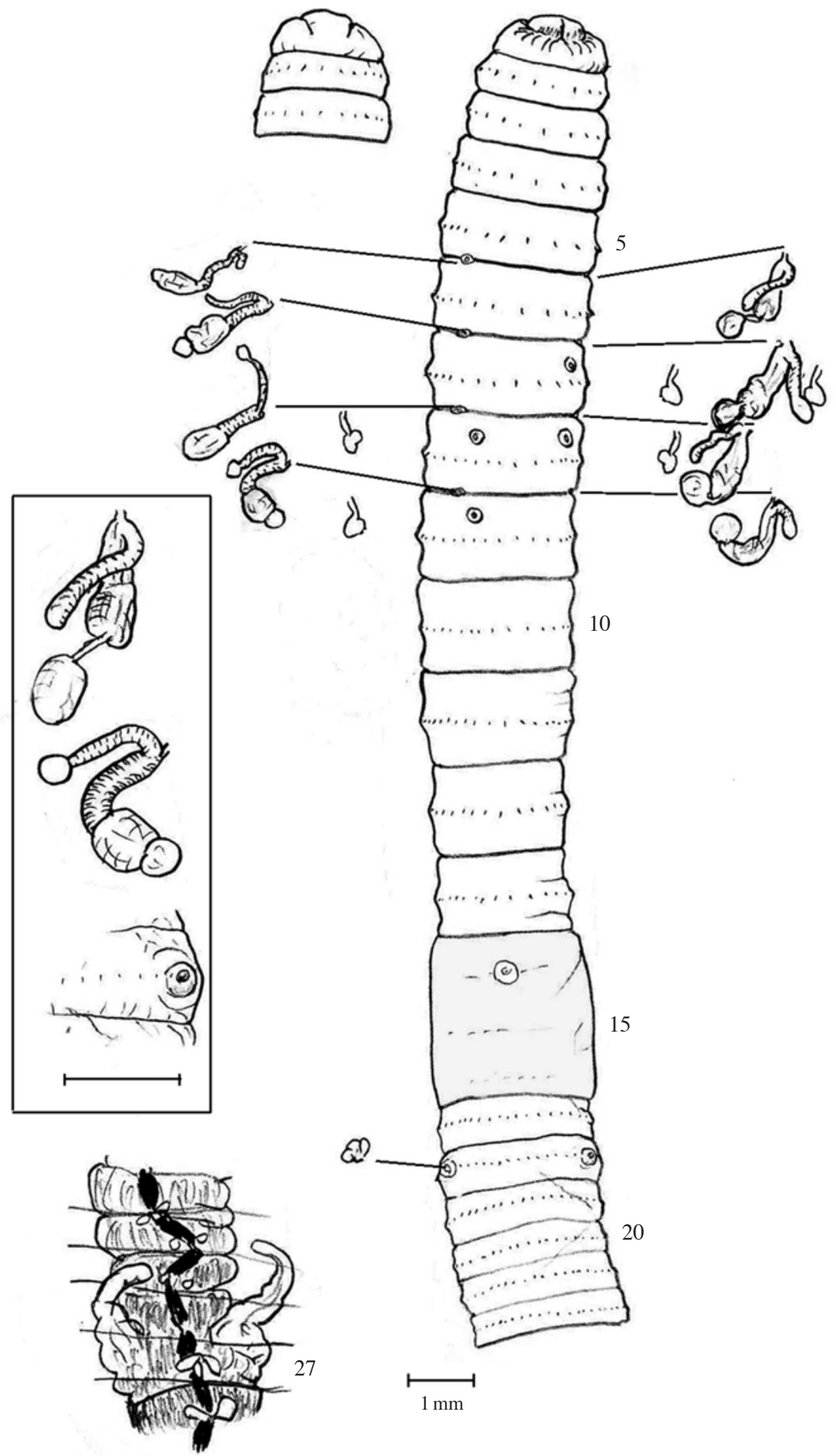

Fig. 7. Amynthas corticis saeseum sub-sp. nov. holotype showing ventral aspect; prostomium, spermathecae (those on lhs from P1), gland in 18 and caeca in situ with [boxed] enlargement of spermathecae from $\mathrm{H}$ and $\mathrm{P} 1$ and of male area 18rhs].

representative of their "hawayanus species-group" while noting that the precise identity of the type-series could not be established as the specimens are immature.

Kinberg's original description, in full, was: " $N$. gracilis n. Lobus cephalicus longitudinem partis mediae superioris segmenti buccalis aequans; segmenta 89-91; longitudo 42-66 mm. Rio de Janeiro, in horto botanico."

The current specimen complies with earlier descrip- 
tions although it lacks the GMs frequently seen in some or all of 6-8,9 near the spermathecal pores in 5/6/7/8 and in groups of up to eleven (or twelve) median to male pores and sometimes on 17 and 19 too (Gates, 1972: 189; Sims \& Gerard, 1999: 130). Without reference to DNA data, this would reasonably be the first record from Korea. However, a potential sub-species is tentatively described below that differs from the more familiar concept of the cosmopolitan A. gracilis, yet its DNA - at least for the part of the COI gene-agrees $100 \%$ with the current specimen. At the same time, the only previously A. gracilis barcodes from Japan seem to indicate slight differences (Appendix). Morphology and DNA are recorded, leaving the outcome of the issue of correct identity and taxonomic relationship of either taxon flagged for future reference.

\section{Amynthas gracilis insularum sub-sp. nov.} [Fig. 8B]

Material examined. IV0000251309, holotype (H) mature specimen sketched and dissected also providing DNA sample (WO55 resampled as w31). Collected from Cheonji-yeon Falls, Seogwipo, 13 ${ }^{\text {th }}$ June, 2012 by RJB.

Etymology. Latin for "of the islands" (latinized genitive plural).

Description. Length $110 \mathrm{~mm}$, segments 86 . Colour light brownish with darker mid-dorsal line, clitellum buff in 14-16. Prostomium epilobous. First dorsal pore in 10/11. Setae, larger and fewer in 4-7,8 (as in A. gracilis proper), number approximately 20 in 5 up to about 60 after clitellum. Spermathecal pores in 5/6/7/8 ca. 0.25-0.3 C. Female pore on 14 . Male pores superficial $0.3 \mathrm{C}$ apart on 18 with approximately 16 setae intervening. Genital markings in tight triplets, posterio-median to male pores on 18 , with corresponding branched glands internally.

Internally, tufted nephridia occur in 5 and 6. Septa 8/9 /10 are aborted around muscular gizzard. Hearts in 10-13. Holandric, but iridescence not noted. Seminal vesicles are in $11 \& 12$ with pseudovesicles in $13 \& 14$. Spermathecal ampulla spherical, duct tapers with ental diverticulum as thin stalk and slightly dilated terminal portions. Oesophagus dilated and vascularized in 11-13, intestine begins in 15 . Caeca single, incised ventrally and dorsally ruggose from ca. 27.

Remarks. Differences from A. gracilis proper possibly are a reduced first segment, dorsal pore more anteriorly in 10/11, slight difference in shape of spermathecae, distinct distribution of genital markings between male pores on 18 (a triumvirate rather than characteristically in series laterally) and caeca that are 'quasi-pinnate', rather than incised on ventral edges. As with the sympatric specimen described above, GMs are lacking between spermathecal pores.
DNA data in Appendix indicates agreement with specimens from Okinawa identified as "Amynthas gracilis" but some slight genetic separation is apparent for other specimens from the Japanese mainland. No other COI data is yet available on GenBank thus the current name awaits confirmation from further data and comparison with the fifteen or so historical names included, in whole or in part, in the nominal taxon's synonymy.

A similar species is Amynthas papulosus (Rosa, 1896) and the closeness of its relationship also requires consideration.

\section{Amynthas hupeiensis (Michaelsen, 1895)}

Remarks. Cosmopolitan species also from Japan and mainland Korea often in or near irrigated rice paddy fields (that were not searched during current Jeju surveys). It was first described from Jeju by Kobayashi (1938: 152) and is fully described by Blakemore (2010b).

\section{Amynthas kanrazanus incretus (Kobayashi, 1937)}

Remarks. Amynthas kanrazanus incretus (Kobayashi, 1937: 343, fig. 4) syn. Amynthas alveolatus Hong \& James, 2001 after Blakemore (2015), from Mt Halla. Amynthas yongshilensis (an A. tokioensis synonym) was also described from Mt Halla, Yongshil, and stated (Hong \& James, 2001: 81) only to be similar to their subsequent $A$. alveolatus yet, remarkably, A. alveolatus - that is an obvious junior synonym of $A$. kanrazanus incretus from the same type-locality - was not compared to any previous species! Moreover, their subsequent species, $A$. geomunensis Hong \& James, 2001: 82, was similarly compared only their two preceding names ignoring similarity to A. kanrazanus.

The sub-species A. kanrazanus incretus was included under its nominal taxon by Song \& Paik (1969: 136, 1970: 10), but this requires further consideration and it is here provisionally restored until a neotype can be selected from topotypes (and DNA extracted).

Taxonomic note. Kobayashi's name Pheretima kanrazana var. increta (cf. Pheretima incerta Beddard, 1912: 197) was misspelled as "incertus" in Sims \& Easton (1972: 237, 243) and this has been copied over to iBOLD, the Barcode of Life and other websites.

\section{Amynthas kanrazanus kanrazanus (Kobayashi, 1937)}

Remarks. Nominal sub-species described from Mt Hala, Jeju (Kobayashi, 1937: 340, fig 3). Amynthas yongshilensis Hong \& James, 2001 is possibly a synonym but is currently (Blakemore, 2010a) held under A. tokioensis. It was claimed on Jeju by Song \& Paik (1969; 1970) but not found in the current survey (cf. A. tokioensis). There 

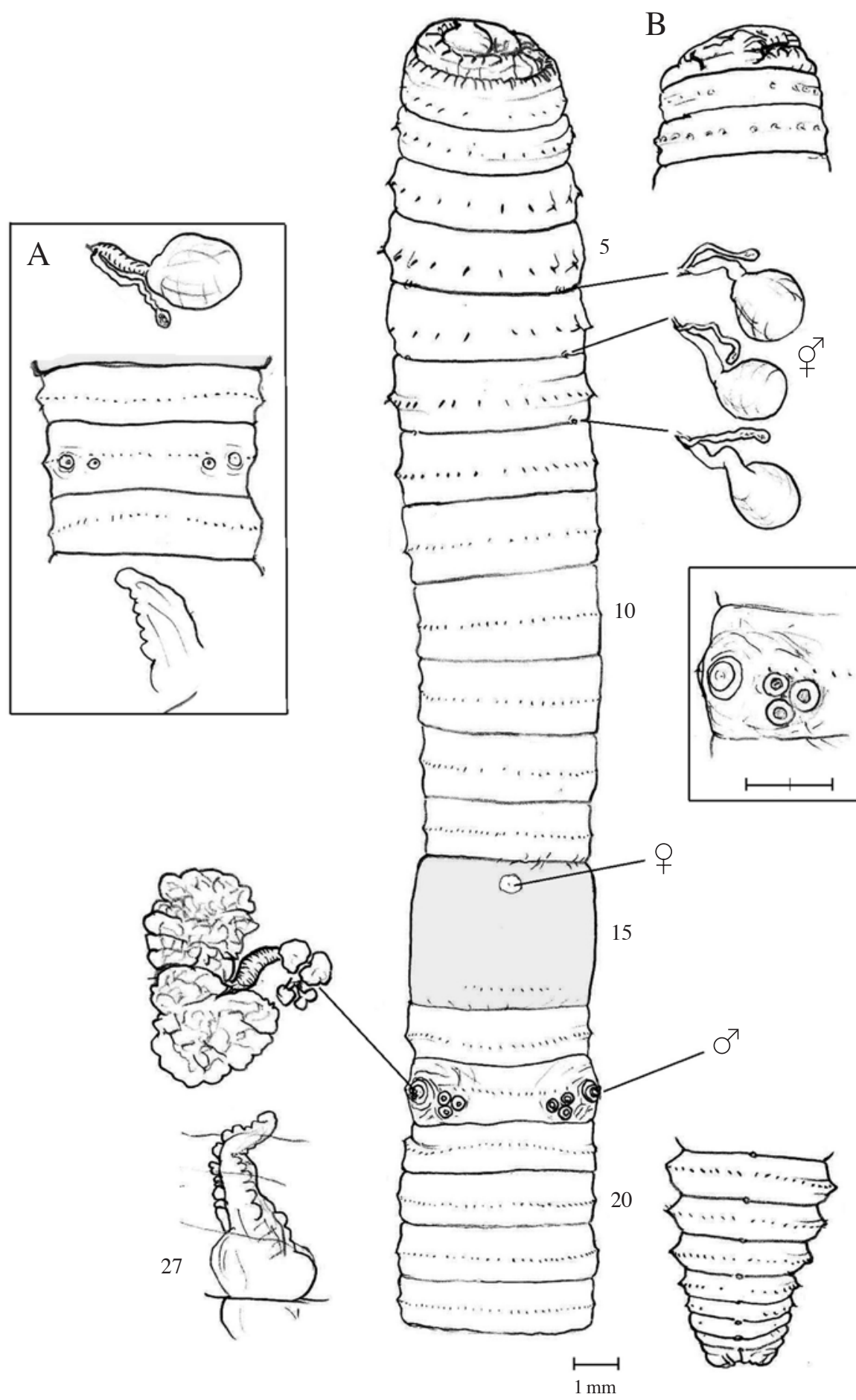

Fig. 8. A. ?Amynthas gracilis gracilis spermatheca from 7rhs, male field and caeca from 27rhs; B. Amynthas gracilis insularum sub-sp. nov. holotype showing ventral aspect; prostomium and pygidium, spermathecae, prostate in situ and caecum in 27 with [boxed] enlargement of male pore $181 \mathrm{hs}$.

seems no reason why it was initially ignored by Hong \& James (2001), nor subsequently during the last dozen years.
Amynthas masatakae (Beddard, 1892)

[Fig. 9A]

Material examined. British Museum lectotype (912) de- 
signated and described by Blakemore (2012d). IV00002 50401 providing DNA samples (WO52 failed to yield results resampled as w28 that was contaminated in the lab but was successfully resequenced as w28b) from Cheonji-yeon Falls collected $13^{\text {th }}$ June, 2012 by RJB.
Remarks. Originally from Japan and described from Jeju by Kobayashi (1937: 337) and Song \& Paik (1970: 11); it was fully redescribed based on its London types by Blakemore (2012d: fig. 1lhs). Results have since arrived for DNA barcode of the topotypic specimen from Nogeyama,

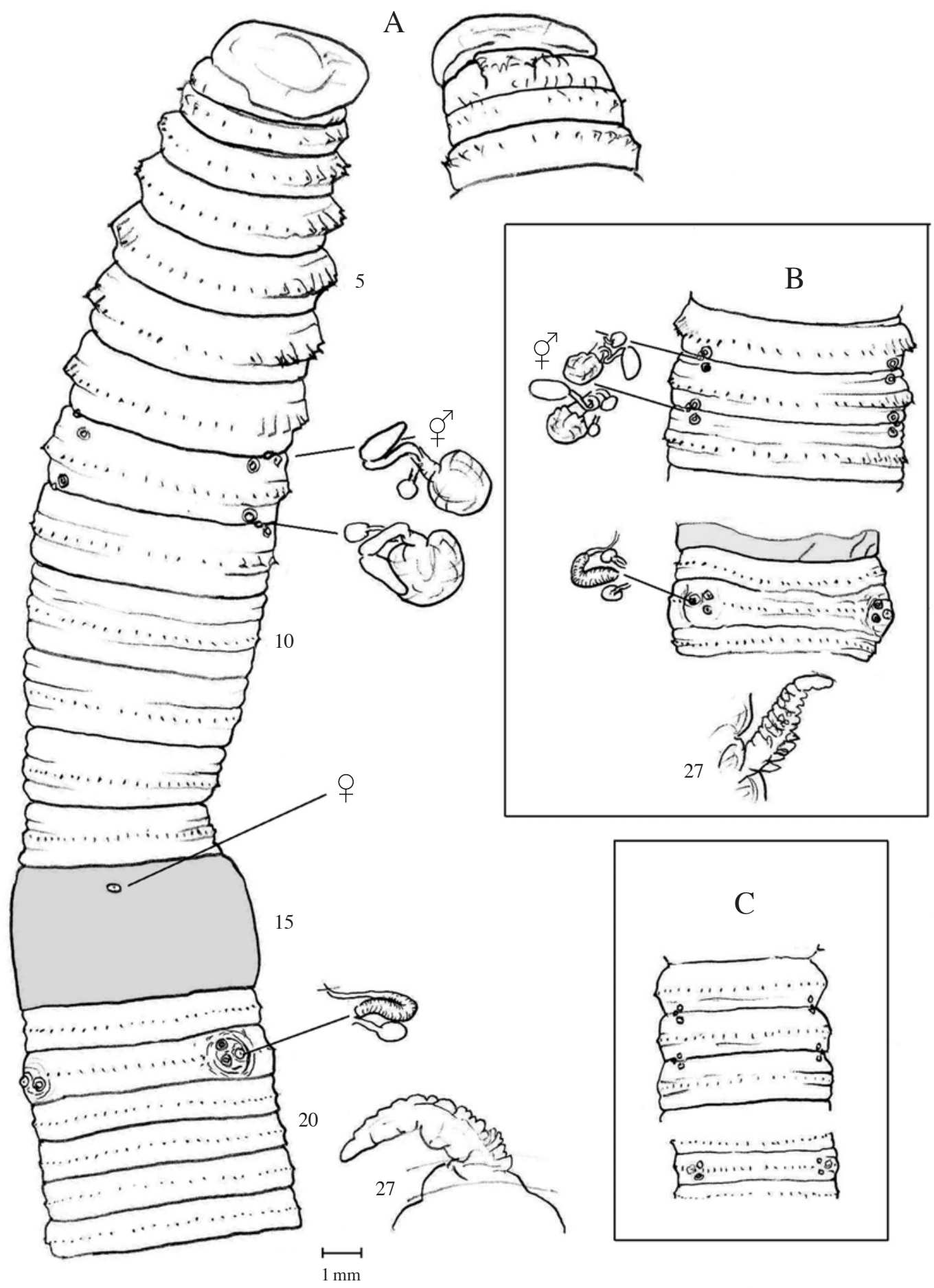

Fig. 9. A. Amynthas masatakae specimen showing ventral aspect; prostomium, spermathecae and prostate duct in situ and caecum in 27; providing DNA (w28b). B. Amynthas tralfamadore showing spermathecae and prostate duct in situ plus caecum in 27 (w29). C. A. tralfamadore second specimen providing DNA (w30). 
Yokohama described in Blakemore (2012d, fig. 1rhs) (IV0000249941 WO35) that agrees 100\% with Cheonjiyeon Falls samples, that now confirm the taxon on Jeju. DNA results for other potential specimens were inconclusive.

megaBLAST comparison indicates $100 \%$ match with EF077538 “A. triastriatus" from China (an erstwhile synonym of $M$. masatakae) that indicates the Chinese specimens are synonyms of $A$. masatakae, however it is not certain that their identification as A. triastriatus (Chen, 1946: 97) proper is correct thus the true synonymy of this name is not proven. Similarly A. robustus (Perrier, 1872) proper is not proven from Japan (nor Taiwan) and these GenBank vouchers should likely be recorded as A. masatakae following revision by Blakemore (2012b). Gates (1972) had A. masatakae in synonymy of A. robustus (Perrier, 1872) and hence included Quelpart in the domain of this latter taxon, now known to be doubtful due to recent work (Blakemore, 2012d), thus a restricted $A$. robustus is excluded.

\section{Amynthas micronarius (Goto \& Hatai, 1898)}

Material examined. Korean specimen (NIBR IV00002 46442) collected from NIBR's Jeju Island Biosphere in Incheon (NIBR IV0000246442 WO1) described by Blakemore (2012d, fig. 6rhs); Jeju Island new specimens (NIBR IV0000250396-7) from Hyomyungsa Temple, Mt Halla collected $12^{\text {th }}$ June, 2012 providing DNA samples (WO48, w26).

Remarks. DNA data confirms a 100\% match (Appendix) showing the Incheon specimens likely originated in Jeju just as the author suspected in the full redescription with revised synonymy provided by Blakemore (2012b: 138). A subsequent possible new synonym (noted by Blakemore, 2012d) is Amynthas montanus Qiu \& Sun, 2012 that has its setae seriously miscounted between descriptions and tables compared to their fig. 2; e.g., 12-18 are described between male pores yet more than 26 are figured, thus setal ranges are given as 52-142 which approach the lower counts from $A$. micronarius synonyms are unreliable. Sun et al. (2012: 149) also misspell their earlier taxa as "trapezoidesi" rather than A. trapezoides Qiu \& Sun, 2010.

\section{Amynthas phaselus maculosus (Hatai, 1930: 661) comb. nov. [Fig. 10]}

Material examined. IV0000261231 mature specimen collected from besides a stream near Sanbansan SW Jeju, $12^{\text {th }}$ June, 2012 by RJB - a mature that was figured and dissected, providing DNA samples (WO51 redone as w27). Included in same jar is a tail portion.

Description. (Jeju specimen). Length $100 \mathrm{~mm}$. Segments
99. Colour brownish, clitellum buff. First dorsal pore 11/ 12. Genital markings absent. Setae ca. 36 on segment 10. Spermathecae ca. 0.3 C apart in 5/6/7/8. Male pores superficial on 18 with 12 setae intervening. Septa 8/9/10 are aborted around gizzard. Spermathecae in 6-8 with elongate and corrugated ampulla on shorter duct with clavate diverticulum. Hearts are in 13. Holandric: testis in $10 \& 11$, seminal vesicles in $11 \& 12$ the latter pair with finger-like dorsal projections. Ovaries in 12, no ovisacs. Prostates large in 18. Oesophagus dilated in 11-13. Intestinal origin in 15 , no typhlosole to about 35 . Gut contains mainly soil.

Remarks. The current specimen agrees with earlier descriptions of Amynthas kamitai (Kobayashi, 1934) from Jeju that appears now to correspond with A. maculosus (Hatai, 1930) from northern Japan, that itself was previously included in synonymy with $A$. phaselus (Hatai, 1930), e.g. by Blakemore (2008). A. maculosus was revived based on a Tokyo syntype by Blakemore (2012: 113, fig. 12). Its new synonyms are Pheretima kamitai Kobayashi (1934: 5, figs. 4-6; 1938: 146, fig. 11) that was from Seoul and latterly included $P h$. serrata Kobayashi (1936: 165, text fig. 10) in synonymy. Referred to as $P h$. phaselus var. kamitai by Kobayashi (1938: 411) and by Song \& Paik (1969: 16, 1970: 11) on specimens from Hokkaido and Jeju, respectively. Since nothing of substance separates Amynthas minjae Hong, in Hong, Lee $\& \operatorname{Kim}$ (2001) from these prior taxa, it too is included in synonymy. Reduced to a sub-species of the nominal taxon, the question of its relationship to A. phaselus is not fully resolved. This Jeju specimen (initially thought similar to A. gracilis) complies with A. maculosus, or rather its synonym $A$. kamitai, more so than with $A$. phaselus proper.

\section{Amynthas phaselus phaselus (Hatai, 1930: 659) [Fig. 11]}

Material examined. IV0000251101, mature posterior amputee specimen collected from Mt Halla $12^{\text {th }}$ June, 2012 from under bridge on Rt 516 that provided DNA (WO42 resampled as w22). An unregistered specimen from Cheonji-yeon collected by RJB on $13^{\text {th }}$ June, 2012 is similar.

Description. (Of Jeju specimen). Length $50+\mathrm{mm}$. Darkish dorsum, clitellum buff. First dorsal pore 12/13. Setae $\sim 60$. Spermathecal pores distinct and nozzle-like in 5/6/ 7/8 ca. $0.3 \mathrm{C}$ apart. Male pores as small lateral slits centred on flat porophore on 18 with ca. 14 setae intervening. No GMs. Nephridia are tufted in 5 \& 6. Septa 8/9/ 10 aborted around gizzard, otherwise thin. Body filled with gregarines, especially segment 12 . Spermathecae in 6-8, corrugated ampulla with shorter duct and digitiform diverticulum. Holandric, testis in large sacs in 10 and free 


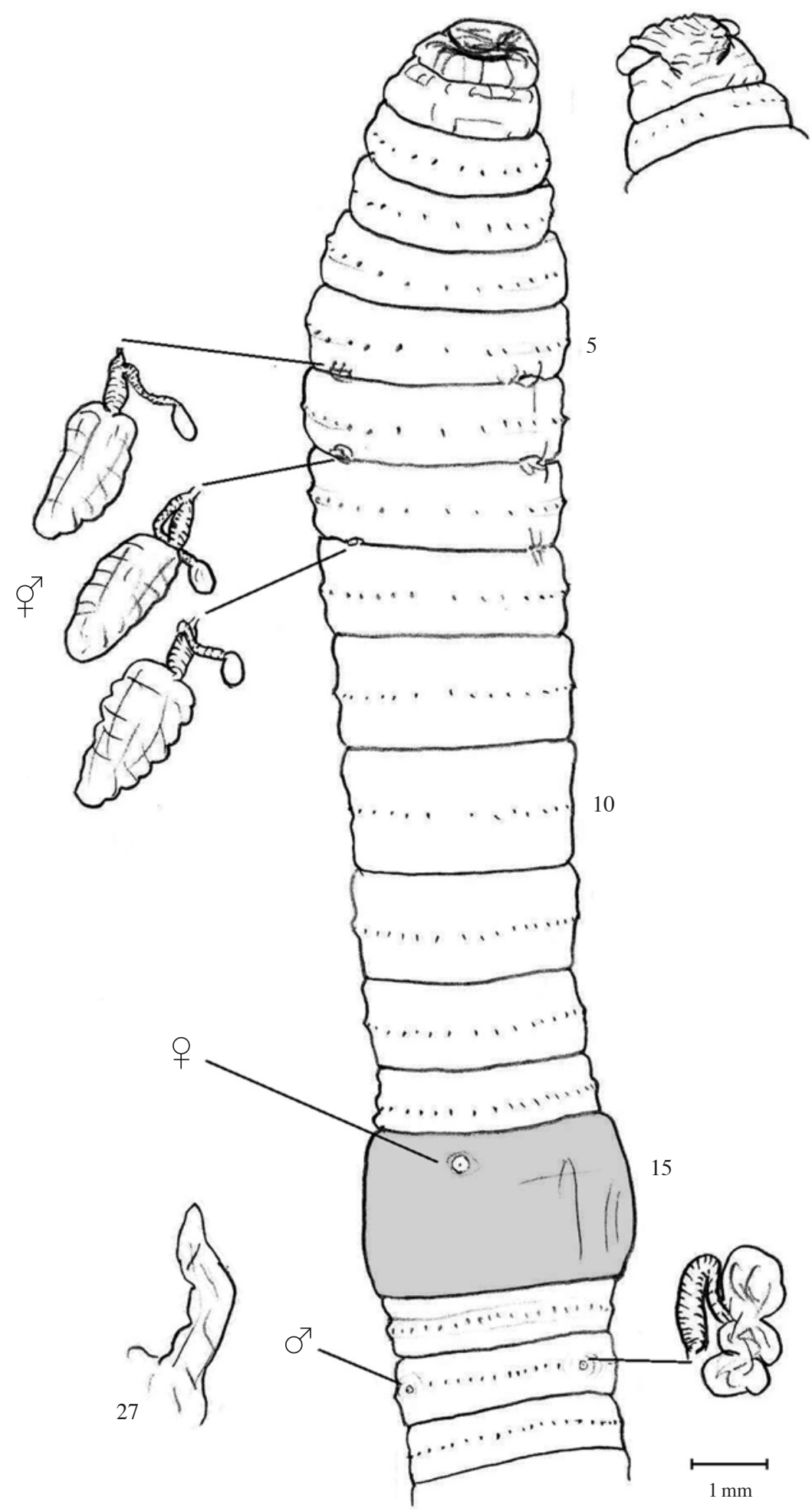

Fig. 10. Amynthas phaselus maculosus (Hatai, 1930: 661). Ventral view with prostomium, spermathecae and prostates in situ; caeca 27rhs.

in 11 , non-iridescent, seminal vesicles anteriorly in 11 \& 12 with pseudovesicles in 13 . Hearts in 10-13. Ovaries in 13 , no ovisacs in 14. Prostates with large glands on flaccid duct in 18 . Oesophagus dilated and vascular in
11-13, intestine from 15, intestinal caeca simple but deeply incised ventrally (hemi-pinnate), typhlosole not noted.

Remarks. Current specimens resemble Amynthas phaselus (Hatai, 1930) that is known from Japan and main- 

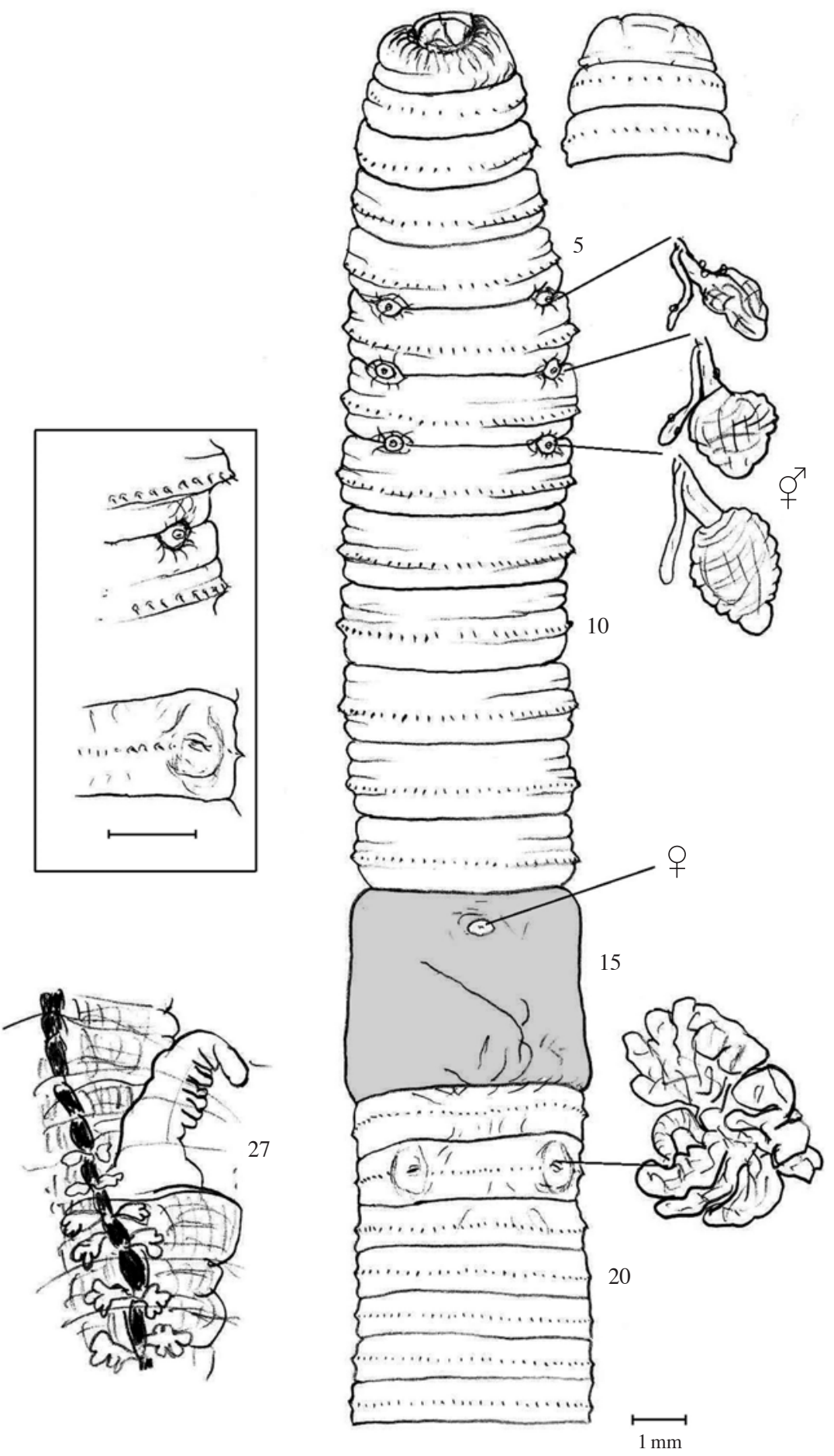

Fig. 11. Amynthas phaselus phaselus (Hatai, 1930: 659). Ventral view with prostomium, spermathecae and prostates in situ; caeca 27rhs shown with lymph glands on dorsal blood vessel; [boxed are enlargemnts of spermathecal and male pore in 7/8rhs and 18rhs].

land Korea and nominally includes synonyms: ?Pheretima maculosus [sic] Hatai, 1930b: 661 (non Pheretima maculosa Gates, 1933=Pheretima malacus Gates, 1936), Pheretima kamitai Kobayashi, 1934, Pheretima serrata Kobayashi, 1936, ?Pheretima phaselus tamurai Kobaya- shi, 1938, Pheretima mutica Chen, 1938 and A. minjae Hong, 2001. Whether A. maculosus and its synonyms (mentioned above) should remain separate or be combined in A. phaselus depends of further work. Currently, only the more "bean-shaped" male pores seem to sepa- 
rate them but confirmation from DNA data is wanting. Blakemore (2012: 113) recorded a possible syntype in Tokyo Museum, while noting that revision is underway based on new material recently collected not only from Jeju but also from Tokyo, from Seoul and on Ulleungdo Island (see Blakemore, 2013). Its full description is pending.

\section{Amynthas sangumburi Hong \& Kim, 2002}

Remarks. Described by Hong \& Kim (2002: 198, fig. 4) from Sangumburi volcanic crater near Mt Halla, it resembles A. toriii (Ohfuchi, 1941: 244) from a cave in Oitaken that is currently in synonymy of $A$. corticis. However, Blakemore (2012b: 143) found this indistinguishable from A. subrotundus (Ishizuka, 2000) itself similar to $A$. rufidulus (Ishizuka, 2000) that were both placed in $A$. corticis complex by Blakemore (2003). Therefore, possibly A. sangumburi also belongs in synonymy either of $A$. corticis or of the Japanese species, otherwise its validity dependent upon deeper research by its original authors. Sometimes misspelt "A. sangumburiensis" (e.g. http:// www.annelids.org/bbta/Main/main_3_2_1.asp). A. sangumburi is compared by Sun et al. (2012: 152, 153, tab. 1) to their A. fuscus that is actually yet another junior synonym of A. carnosus as already clarified above. A similar taxon is A. simplex sp. nov. described below.

\section{Amynthas seungpanensis (Song \& Paik, 1970)}

Remarks. Amynthas seungpanensis (Song \& Paik, 1970: 11, figs. 1-13) described from "Mt Hanra, Chejoo-do Isl.", has not been seen since. Its definitive characteristics were supposed to be spermathecal pores intrasegmental on 6 and 7 and distinctive male pores. Its name is sometimes misspelt "Ph. seungpanesis". "Mt Hanra", or Mt Hanla amongst its ten or so previous names, is now known as Mt Halla.

\section{Amynthas simplex sp. nov.}

[Fig. 12]

Material examined. IV0000251102 Holotype (H) mature specimen from Mt Halla under bridge on Rt 516, $12^{\text {th }}$ June, 1023; providing DNA sample (WO43 resampled as w23). Paratype (P) in same jar, undissected.

Etymology. Latin for "simple" due to its lack of GMs and other complex attributes (Non Michaelsen, 1928).

Description. Length $80 \mathrm{~mm}(\mathrm{H}), 60 \mathrm{~mm}(\mathrm{P})$. Segments $100(\mathrm{H}), 101(\mathrm{P})$. Darkish dorsum but transparent ventrum when preserved with internal parasites visible, clitellum buff and rather knobbly (H \& P) possibly due to parasitism. First segment reduced with thin and wide epilobous prostomium. First dorsal pore minute in $12 / 13$, open from
13/14 (H \& P). Spermathecal pores ca. $0.3 \mathrm{C}$ apart in 5/ $6 / 7 / 8 / 9$. Setae ca. 24ish per segment with ventral gaps in anterior; the penultimate three segments of 'tail' lack setae. No GMs. Male pores small in centres of large circular flat porophores. Nephridial forests in $5 \& 6$. Septum 8/9 aborted, 9/10 displaced by gizzard. Holandric with large testis sac in 10 and seminal vesicles in $11 \& 12$. Hearts 10-13. Ovaries in 13, no ovisacs present. Oesophagus vascularized and dilated in $12 \& 13$. Intestine from 15. Intestinal caeca simple. No typhlosole. Gut contains organic soil. Some large nematodes and much mucus seen in anterior segments, with many gregarine cysts, especially on ventral nerve cord under intestine.

Remarks. Similarities to A. phaselus are lack of GMs, ruggose spermathecae and simple, circular male fields. Differences are an extra pair of spermatheca in 8/9 and non-incised intestinal caecae. The other similar Jeju species with spermathecae in 5/6-8/9 and simple caeca is Amynthas sangumburi Hong \& Kim, 2002 that, however, is smaller (46-68 mm), has more setae (39-43 with only 3-4 between male pores) and quite different shape of spermathecae plus a large typhlosole from 27 . These specimens are named as new on the basis of these morphological differences that, unfortunately, could not yet be confirmed by DNA.

\section{Amynthas tokioensis (Beddard, 1892)}

Material examined. [IV0000250398 a sub-adult specimen from Hyonyungsa Temple (12 $2^{\text {th }}$ June, 2012, RJB) that provided DNA sample (WO49 detailed in Appendix) complying with either (or both?) A. tokioensis and Metaphire hilgendorfi using megaBLAST searches].

Remarks. Amynthas tokioensis (Beddard, 1892) [syns. ?schizopora, ?irregularis, levis, ?parvicystis; ?verticosa; ?yongshilensis Hong \& James, 2001, ?eastoni Hong \& James, 2001; ?boletiformis Hong \& James, 2001 (these last two synonyms as per Blakemore, 2003: 43, addenda); ?gucheonensis Song \& Paik, 1970; jiriensis Song \& Paik, 1971; ?paiki Hong in Hong, Lee \& Kim, 2001: 266 (these last three synonyms as per Blakemore, 2006; 2008)]. Plus ?Pheretima gucheonensis Song \& Paik, 1971, ?Pheretima surcata Ishizuka, 1999 and A. sonjaesiki Hong \& James, 2009 were placed in synonymy of A. tokioensis by Blakemore (2010a: 13, fig. 2) based on reinspection of its London types. Blakemore \& Ueshima (2011) \& Blakemore (2012d, f) described syntypes of Amynthas levis (Goto \& Hatai, 1899) as being similar to a parthenogenetic form of A. tokioensis and thus a possible synonym, as was already noted by Blakemore (2012b).

Amynthas conferticurtus Hong \& James, 2009: 1241 appears to be misdescribed in several key characters: e.g., the spermathecal pores are said to be on 7 and 8 (thus allegedly qualifying for an A. pomellus species group; 


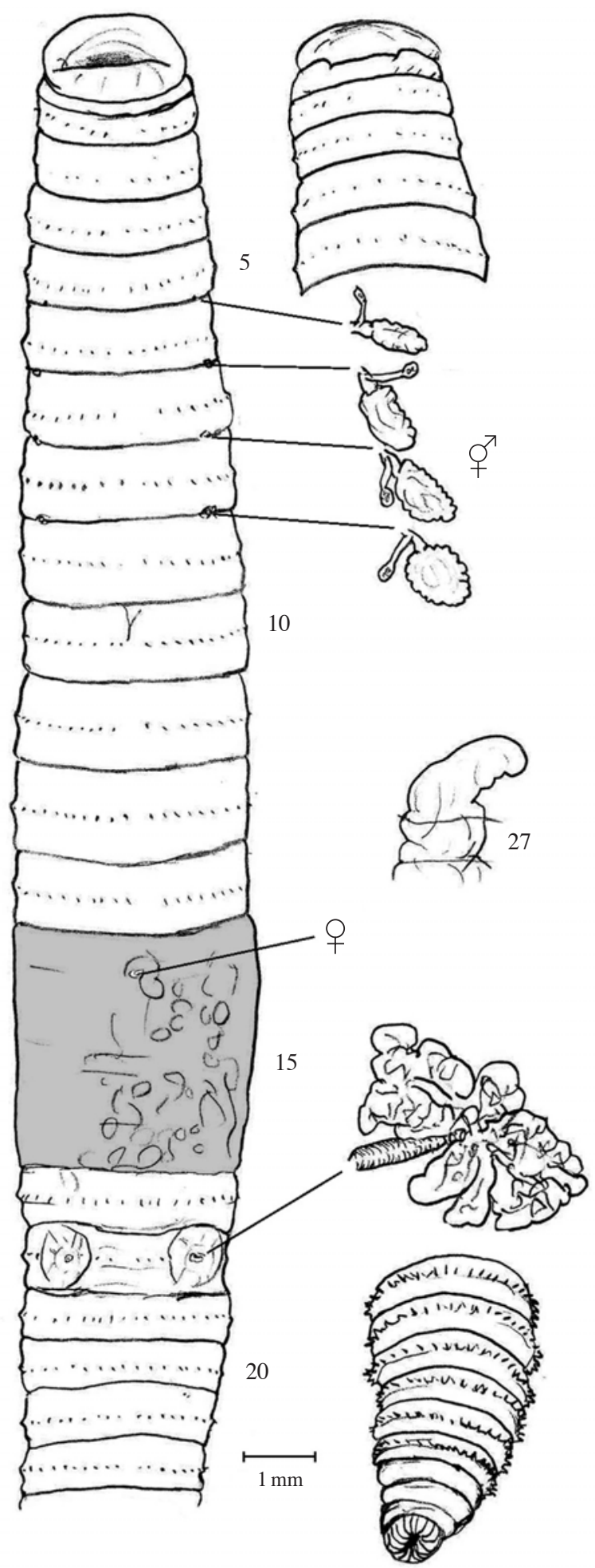

Fig. 12. Amynthas simplex sp. nov. H. Ventral view with prostomium and pygidium, spermathecae and prostates in situ; caeca 27rhs.

however, they are shown in their fig. 7 to be in $6 / 7 / 8$ ! Moreover the supposed genital markings on $7 \& 8$ and
18 are not shown in their figure 7 ! Their description with this new name appears to be indistinguishable from the subsequent A. sonjaesiki Hong \& James, 2009: 1243 that has markings resembling those claimed for A. conferticurtus and is moreover similar to A. paiki Hong, 2001 and/ or to A. fasciiformis Hong \& James, 2001, thus both latter names are probably in an A. tokioensis species-group, if not also synonyms. It seems that the A. conferticurtus name also belongs.

A. tokioensis is included due to record from Jeju of the likely synonym A. yongshilensis Hong \& James, 2001; cf. Metaphire hilgendorfi noted below.

Note. Regarding types, Amynthas conferticurtus Hong \& James, 2009 with types IV0000120468 (H) \& 479 (P) not traceable in NIBR, its synonym, A. sonjaesiki Hong \& James, 2009 with types IV0000120469 (H) \& 480 (P) also not traceable in NIBR.

\section{Amynthas tralfamadore Blakemore, 2012} [Fig. 9B, C]

Material examined. Type specimen (NIBR IV0000246 441) collected from NIBR's Jeju Island Biosphere at Incheon were described by Blakemore (2012d). New specimens (IV0000250402-3) three mature specimens sketched and dissected, providing DNA samples (WO53-54 resampled as w29-30) collected outside of Cheonji-yeon Falls Park, Jeju on 13th June, 2012 by RJB from under rocks and beside a spring.

Remarks. These specimens confirm, as anticipated, both separation of A. tralfamadore from A. masatakae and the provenance of both from Jeju suggested by Blakemore (2012d: 143).

\section{Metaphire agrestis (Goto \& Hatai, 1899)}

Material examined. IV0000250406-7 Cheonji-yeon Falls, Seogwipo, collected $13^{\text {th }}$ June, 2012 by RJB; two mature specimens, one sketched and dissected, providing DNA sample (WO56 mixed in lab and resampled as w32 in Appendix). Seven similar mainly immature specimens collected from Cheonji-yeon Falls on $14^{\text {th }}$ June, 2012 (undissected).

Remarks. These specimens conform to the redescription by the current author from Japan and Korea. A neotype in Tokyo Museum is in process of description as noted by Blakemore (2012b; 2012f). Further work is currently in progress to define this cosmopolitan species.

\section{Metaphire californica (Kinberg, 1867)}

Material examined. NIBR IV0000246440, found beside Temple at Mt Sanbangsan, Jeju, collected by R.J. Blakemore, $17^{\text {th }}$ Feb. 2012, from near drainage ditch and described Blakemore (2012: fig. 2); IV0000250461, single 
specimen from Saeseum Island, Seogwipo, collected $13^{\text {th }}$ June, 2012 by RJB, providing DNA (WO58 that was probably mixed with WO57 see Appendix). Cheonji-yeon Falls, $13^{\text {th }}$ June, 2012 collected by RJB (four specimens undissected).

Remarks. This was the first record from Jeju and Korea of this cosmopolitan species (Blakemore et al., 2012).

\section{Metaphire haenyeo sp. nov.}

[Fig. 13]

Material examined. IV000246445 Holotype (H) mature, sketched and dissected providing DNA samples (WM12 redone as w5); collected from Sehwa Beach near to Haenyeo Museum, $16^{\text {th }}$ Feb., 2012 by RJB under rocks on machair. IV0000249896-7 Paratypes (P1-2) two mature specimens, inspected.

Etymology. Due to location, near The Jeju Haenyeo Museum named after the Jeju diving ladies called 'haenyeo' (noun in apposition).

Description. Length 140 mm (H), 120 (P1), 95 (P2); segments 109 (H, P1). Colour a light green in life, soon bleached out in alcohol. Clitellum weakly formed. Epilobous. First dorsal pore in 12/13 (H, P1) or 11/12 (P2). Setae number about 60-66 per segment. Spermathecae in wide slits in 6/7/8/9 about $0.3 \mathrm{C}$ apart. Female pores paired on 14. Male pores in small c-shaped pouches with 15-17 setae intervening, everted in P2. No genital markings present but small glands noted associated with each spermathecae posteriorly.

Internally, nephridial forests fill 5 and 6. Septa 5/6/7/8 are thin, 8/9/10 are absent in position of muscular gizzard, and 10/11-13/14 have some thickening. Dorsal blood vessel single, hearts in 10-13. Spermathecae with large oval ampulla on short duct and medium diverticulum with inseminated terminal bulb having irregular outline. A small gland associated with each spermatheca ectally. Holandric, with iridescent testis small in 10 \& 11, seminal vesicles in $11 \& 12$. Prostates on thick duct to small copulatory pouch. Ovaries small in 13, no ovisacs in 14. Oesophagus slightly ditated in $12-15$, intestine from 16 , with caeca broadly based from 27 and deeply incised ventrally. Typhlosole not well developed, gut contains organic soil and sand grains. No parasites were noted.

Remarks. Complying with the M. houletti species-group of Sims \& Easton (1972) that included indigo Ohfuchi, 1951, keishuensis Kobayashi, 1938 and quelparta Kobayashi, 1937. Perhaps it is closest to the latter taxon both morphologically and geographically. Similarity is their characteristic greenish blue dorsum and light blue ventrum. Differences in M. quelparta are that the spermathecal and male pores have saccular bodies internally that may protrude and the former are accompanied with paired short stalked accessory glands. An apparently ma- jor difference is that only one, rather than a pair of glands accompanies the spermathecae in the current species that, nevertheless, may only merit sub-specific status, cf. $M$. quelparta. DNA data are provided and compared in the Appendix.

Metaphire quelparta valhalla sub-sp. nov. from Mt Halla appears to differ morphologically, but has similar DNA. Another taxon is M. q. seogwipo sub-sp. nov. that appears to differ molecularly from $M$. haenyeo and morphologically from M. quelparta quelparta. At the same time, no confirmed specimens of $M$. quelparta have been found in the current surveys and thus neither a neotype nor its DNA libarary can be provided presently.

\section{?Metaphire hilgendorfi (Michaelsen, 1892)}

Material examined. [IV0000250398 a sub-adult specimen from Hyonyungsa Temple (12 ${ }^{\text {th }}$ June, 2012, RJB) provided DNA sample (WO49 details in Appendix that gives some slight indication cf. A. tokioensis noted above].

Remarks. Despite the specimen providing the DNA being not fully mature, its DNA should be definitive. However, the GenBank voucher library gave unreliable results and, moreover, there is the slight possibility that this sample was mixed in the genetics lab. Thus this species record is only tentative for Jeju and requires confirmation from resurvey.

\section{Metaphire quelparta quelparta Kobayashi, 1937}

Material examined. A possibly similar specimen is IV0 000250892 from beside Temple at Mt Sanbangsan, Jejudo collected by RJB $17^{\text {th }}$ Feb. 2012 from near drainage ditch that provided DNA samples (WM13, WO11 and w6). Types are not known.

Remarks. Not definitely identified in current studies as the similar specimen found was damaged and thus was not fully studied. Yet Metaphire quelparta was said to be "the most common species on this small volcanic island" (Kobayashi, 1937: 35, fig. 5) and it was also claimed on the Korean peninsula (Kobayashi, 1938: 155) where it was initially mistakenly labelled as "Pheretima keishuensis" nom. nudum by Kobayashi (1938: 90), as noted by Song \& Paik (1970: 11).

In discussing Metaphire yamadai (Hatai, 1930) neotype, Blakemore (2012b: 119) said that, apart from its nonmanicate caeca, "M. quelparta is almost exactly the same in each described character except for its large saccuar bodies associated with the spermathecal pores. But it may belong in synonymy nevertheless, along with $M$. sanseiana (Hatai, 1951: 56), and the probable syn. nov. of the latter species, M. indigo (Hatai, 1951: 58)" and another possible synonym is $M$. sanseiana (Ohfuchi, 1951) from China. Blakemore (2010a: 18, 2012b: 117) reviewed $M$. 


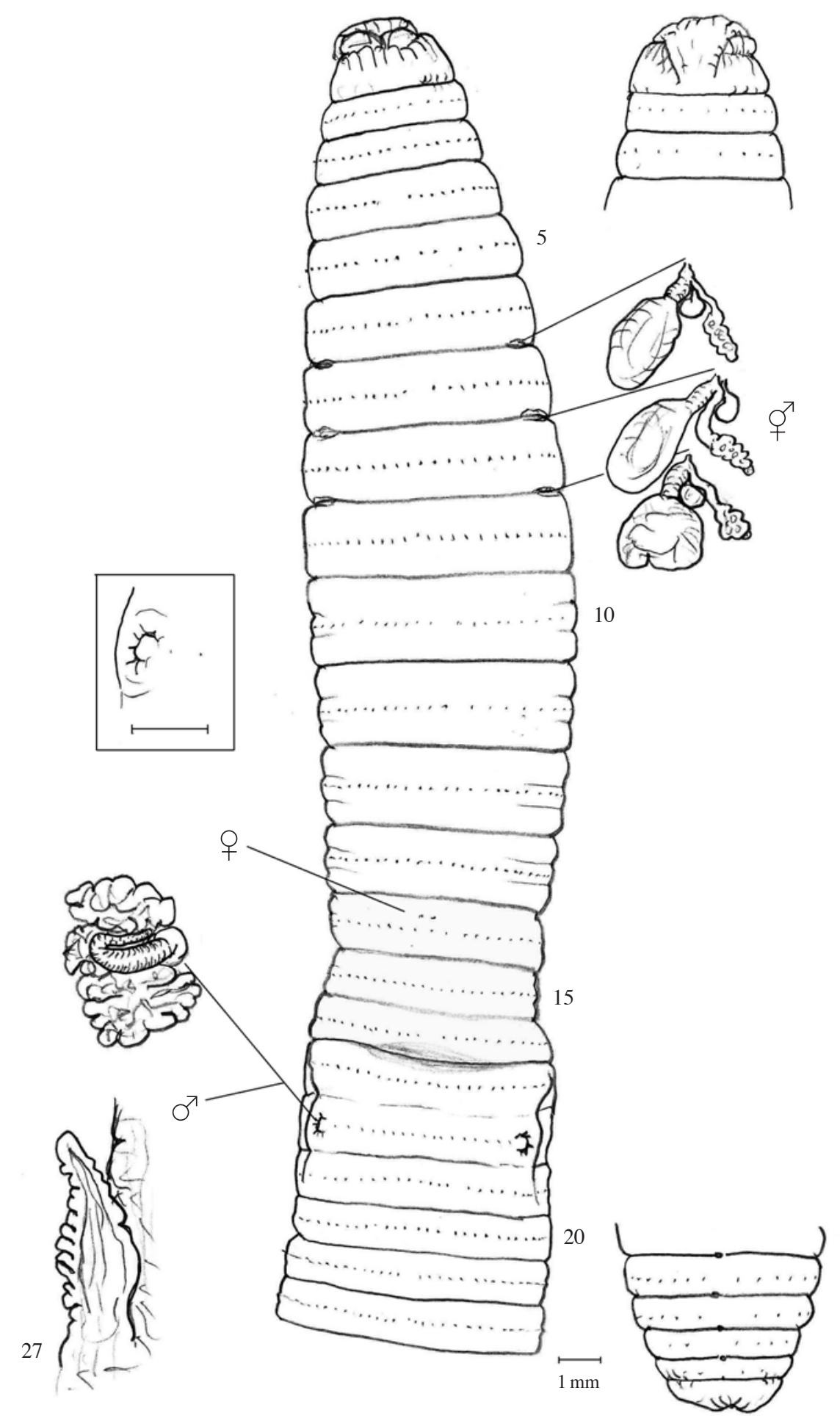

Fig. 13. Metaphire haenyeo sp. nov. holotype showing ventral aspect; prostomium and pygidium, spermathecae, prostate and caecum in situ with [boxed] enlargement of male pore 18lhs].

yamadai, latterly based on Tokyo Museum neotype and noted that for M. yamadai from China, Chen (1933: 259, figs. 20,21) shows variations with the caeca either deeply incised or manicate, but this was likely a misdescrip- tion by Chen of a composite of both Michaelsen's Pheretima pectenifera and Ph. tschiliensis. This latter taxon may indeed have incised caeca and is currently classified as ?Metaphire tschiliensis (Michaelsen, 1928), but further 
research is required from China to confirm its full relationships.

Kobayashi $(1938: 155,157)$ had said that his Ph. aggera Kobayashi, 1938: 153 was close to and may be synonymous with some of the species above and with his $P h$. quelparta. However, Easton (1981)'s inclusion of Metaphire soulensis (Kobayashi, 1938) in synonymy of $M$. yamadai (Hatai, 1930) is no longer supported as there are notable differences in morphology (pers. obs.), especially of the markings around the male pores (when present). Thus, M. soulensis is thus maintained separately and has M. shinkeiensis (Kobayashi, 1938), M. aokii (Ishizuka, 1999) and Amynthas dageletensis Hong \& Kim, 2005 included as its junior synonyms, as clearly stated previously by Blakemore (2003; 2010a; 2012a).

\section{Metaphire quelparta seogwipo sub-sp. nov.} [Fig. 14]

Material examined. IV000261223 Holotype (H) semimature specimen, sketched and dissected providing DNA samples (WO66); Collected $9^{\text {th }}$ Sept. 2012 by RJB, during a short walk from the Botanic Gardens to IUCN meeting at Seogwipo $\left(33^{\circ} 15^{\prime} 10^{\prime \prime} \mathrm{N} 126^{\circ} 33^{\prime} 40^{\prime \prime} \mathrm{E}\right)$ from under rocks beside road.

Etymology. Based on location: Seogwipo (noun in apposition).

Description. Length $130 \mathrm{~mm}$; segments 93. Colour greenblue with dark mid-dorsal line in life. Paler in alcohol. Clitellum weak in 14-16. Epilobous. First dorsal pore in $12 / 13$. Setae number 50-60. Spermathecae in $6 / 7 / 8 / 9$ about $0.3 \mathrm{C}$ apart. Female pore on 14. Male pores in small $\mathrm{C}$-shaped pouches with 12 setae intervening. No genital markings.

Internally, nephridial in 5 \& 6. Septa 8/9/10 aborted around muscular gizzard. Hearts in 10-13. Spermathecae, probably not fully formed, with saccular ampulla on longer duct, and with elongate uninseminated diverticulum that is straight rather than folded. A small stalked gland associated with spermatheca in $7 \& 8$ ectally (not in 9 nor near prostates). Holandric, with iridescent testis in small sacs in $10 \& 11$ posteriorly, seminal vesicles in 11 \& 12 anteriorly. Prostates on thick duct. Ovaries small in 13, ovisacs absent. Oesophagus dilated and vascularized in 13; intestine from $1 / 215$; caeca from 27 incised ventrally. Typhlosole not noted.

Remarks. Although possibly not fully mature, apparent differences from the nominal taxon are lack of noticeable saccular bodies on male and spermathecal pores, and absence of paired spermathecal glands that are shown consistently in Kobayashi's work. Moreover, only a dozen setae intervene between male pores rather than 18-21 as in M. quelparta proper. It is somewhat similar, but differs from M. haenyeo, not least by having longer sperma-

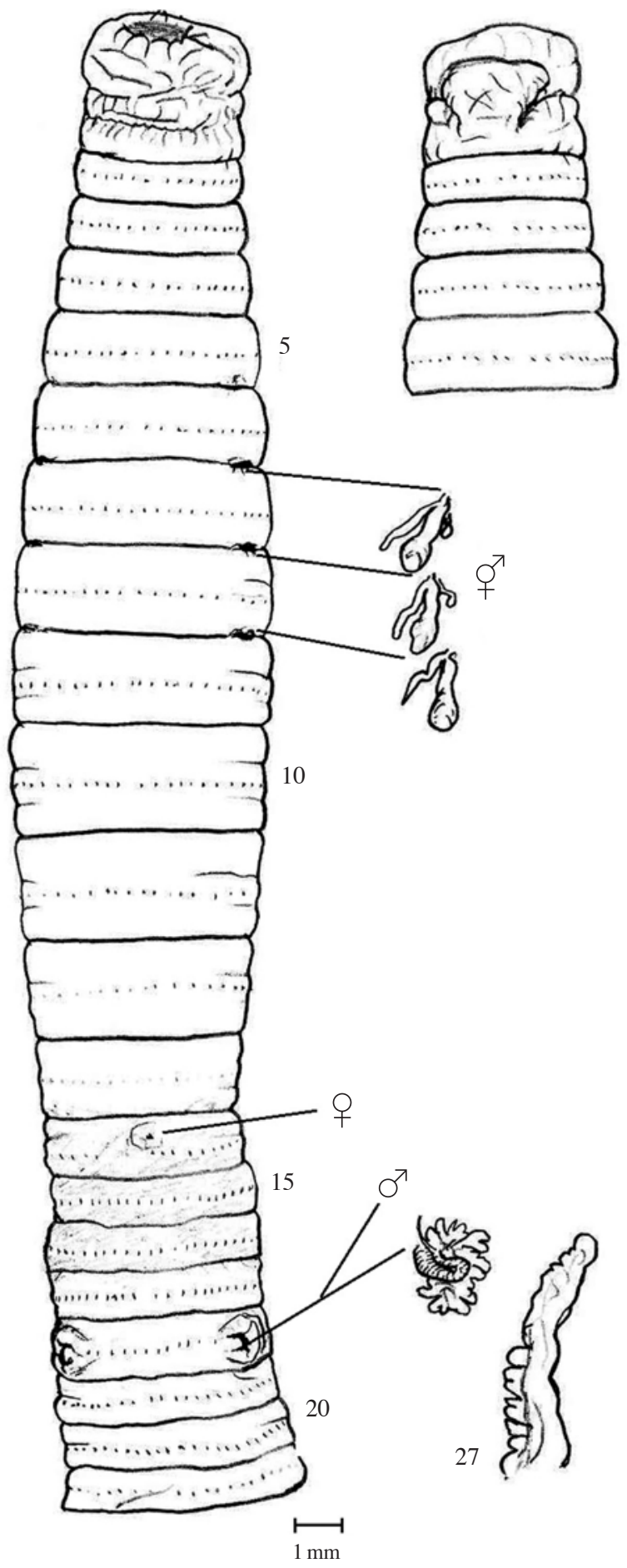

Fig. 14. Metaphire quelparta seogwipo sub-sp. nov. holotype showing ventral aspect; prostomium, spermathecae, prostate and caecum in situ.

thecal diverticula although DNA data indicates slight $(\sim 3 \%)$ separation.

Full relationships require comparison with new material of nominal M. quelparta which had type-localities 
in Seogwipo as well as Jeju-township and Mt Halla listed by Kobayashi (1937). So far such material has been elusive although both the current and subsequent subspecies come closest.

\section{Metaphire quelparta valhalla sub-sp. nov.} [Fig. 15]

Material examined. IV0000251099 Holotype (H) mature specimen, sketched and dissected providing DNA samples (WO40 resampled as w20); collected from Mt Halla under Bridge on Rt 516, 12 $2^{\text {th }}$ June, 2012 by RJB, from under a large rock of valley beside creek.

Etymology. Based on location: a valley on Mt Halla (noun in apposition).

Behaviour. sluggish and non responsive to touch.

Description. Length $250 \mathrm{~mm}$; segments 112. Colour white, unpigmented with distinctive blue dorsal blood vessel and guts visible in life. Clitellum pale too. Epilobous. First dorsal pore in 12/13. Setae number about 60 per segment on 12, 64 on 20; those on ventrum more pronounced in anterior. Spermathecae obvious in 6/7/8/9 about $0.25 \mathrm{C}$ apart. Female pores paired on 14. Male pores in small pouches with 14 setae intervening. No genital markings present but small glands noted behind each spermatheca posteriorly.

Internally, nephridial forests on $4 / 5 / 6 / 7$. Septa 8/9/10 aborted around muscular gizzard, otherwise muscular in anterior. Dorsal blood vessel single, hearts in 10-13. Spermathecae with saccula ampulla, often flattened on medium duct and long diverticulum with inseminated ental half that zig-zags. A small stalked gland associated with each spermatheca ectally. Holandric, with iridescent testis small in 10 \& 11 , seminal vesicles in 11 \& 12 anteriorly and pseudovesicles in 13. Prostates on thick duct to small presumably eversible pouch. Ovaries in 13, ovisacs vestigial in 14. Oesophagus slightly dilated and vascularized in 12 \& 13; intestine from 15; caeca from 27 deeply incised ventrally. Typhlosole not noted.

Remarks. The current sub-species differs from the nominal taxon on several points, especially lack of noticeable saccular bodies on male and spermathecal pores. It is approximately twice the size and lacks the distinctive green colouration. It is somewhat similar, but differs from $M$. haenyeo, not least by having longer spermathecal diverticula although DNA data indicates only slight separation $(\sim 1 \%)$.

Complete comparison with $M$. quelparta depends upon discovery of specimens that comply with Kobayashi's description having green colouration and paired spermathecal glands shown in Kobayashi (1937: figs. 5E-G; 1938: figs. $14 c \& c^{\prime}$ ). Specimens claimed by Song \& Paik (1970: 11) were not described in any detail, they merely their presence on "Chejoo-do" was noted.

\section{Metaphire soulensis (Kobayashi, 1938)}

Remarks. Song \& Paik (1970: 11) also located Metaphire soulensis (Kobayashi, 1938) on "Chejoo-do". Its synonyms are Pheretima shinkeiensis Kobayashi, 1938; Ph. aokii Ishizuka, 1999 - synonymy as per Blakemore (2003: 43) plus A. dageletensis Hong \& Kim, 2005 from Ullungdo. Revision is currently in progress based on Seoul and Ulleungdo specimens (Blakemore, in prep.).

\section{Pontodrilus litoralis (Grube, 1855)}

Material examined. IV0000245076 four specimens as newly reported on Jeju by Blakemore et al. (2012) and it was subsequently confirmed by the author abundantly in Seogwipo port $22^{\text {nd }}$ May, 2012 (unpublished data). One of the latter provided DNA (WO39 that was mixed in the genetics lab. with WO38).

Remarks. Blakemore (2007; 2010b) lists its many synonyms and pantropical distribution.

\section{Lumbricidae}

Aporrectodea caliginosa (Savigny, 1826)

Material examined. IV0000249913 mature specimen from Hamdeok Sewoobyong beach collected $15^{\text {th }}$ Feb., 2012 by RJB providing DNA sample (WO17 that gave ambigous result).

Remarks. Cosmopolitan species, this the first record from Jeju.

\section{Aporrectodea trapezoides (Dugès, 1828)}

Material examined. Mature specimen from Hamdeok Sewoobyong beach collected $15^{\text {th }}$ Feb., 2012 by RJB providing DNA sample (WO16), as yet inconclusive/mixed (cf. WO7 \& WO13).

Remarks. Cosmopolitan species, first recorded on Jeju by Kobayashi (1941).

Bimastos parvus (Eisen, 1874)

Material examined. Jeju from Hamdeok Sewoobyong beach, under logs and stones behind shop fronts unregistered samples with clitellum 24-30, one providing DNA (WO13 currently inconclusive and probably mixed with WO16 or some other sample in lab.).

Remarks. Cosmopolitan species, this the first record from Jeju.

\section{Dendrobaena sp.?}

Material examined. IV0000249923 a mature specimen collected from Hyomyungsa Temple on Mt Halla $16^{\text {th }}$ 

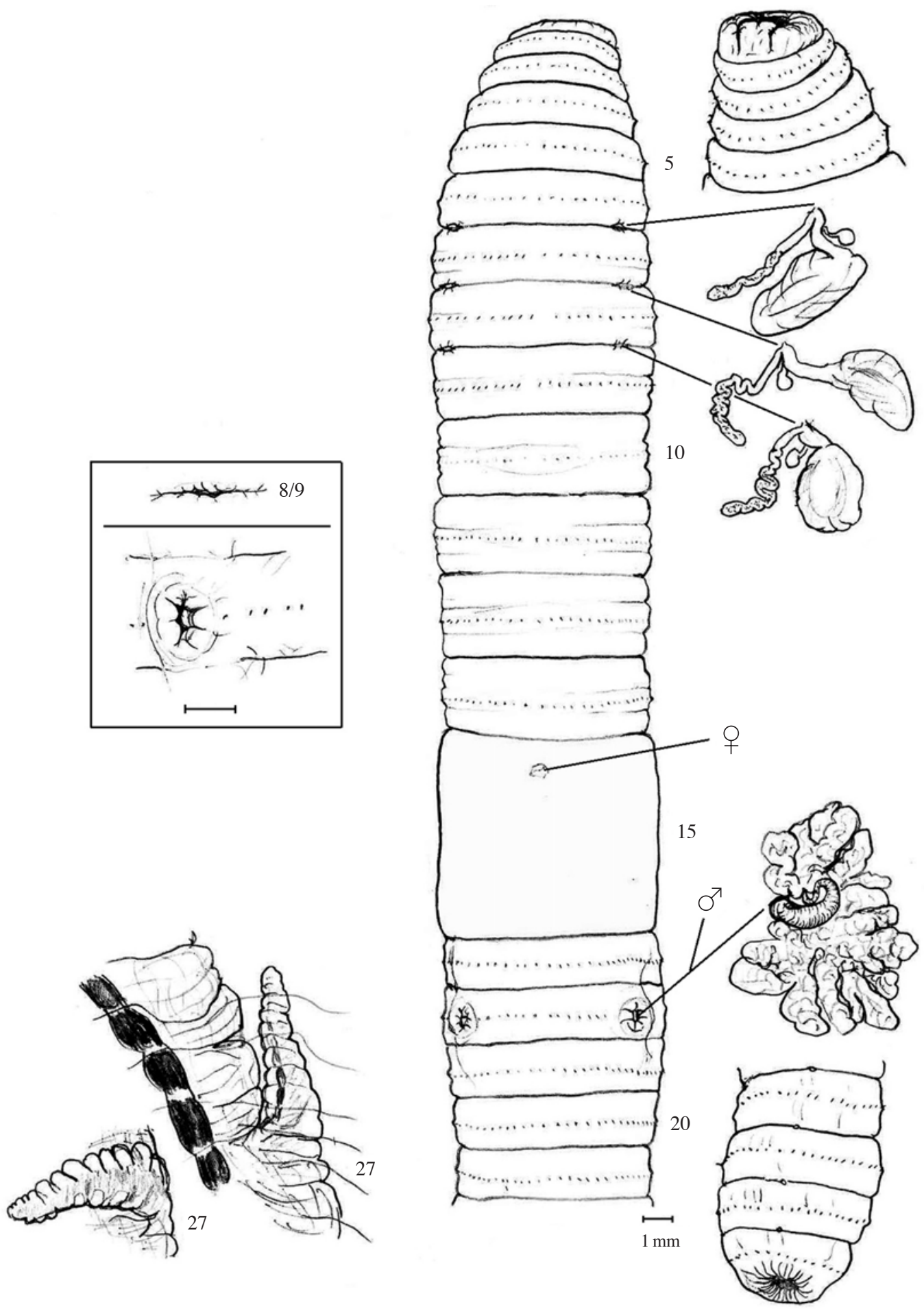

Fig. 15. Metaphire quelparta valhalla sub-sp. nov. holotype showing ventral aspect; prostomium and pygidium, spermathecae, prostate and caecum in situ and folded back with [boxed] enlargement of spermathecal pore and male pore 18lhs.

Feb., 2012 by RJB (providing DNA sample WM10 as "Dendrobaena cf. attemsi" that was mixed in the lab with specimen of E. japonica from NZ in Blakemore (2012c); redone as $w 1$ that has yet to provide a result).
Remarks. Pale lumbricid, $62 \mathrm{~mm}$ long, with segment 9 tumid laterally and 16 ventrally; clitellum $27-33$, with tubercula pubertates wide in 29-32. Currently unidentified. 

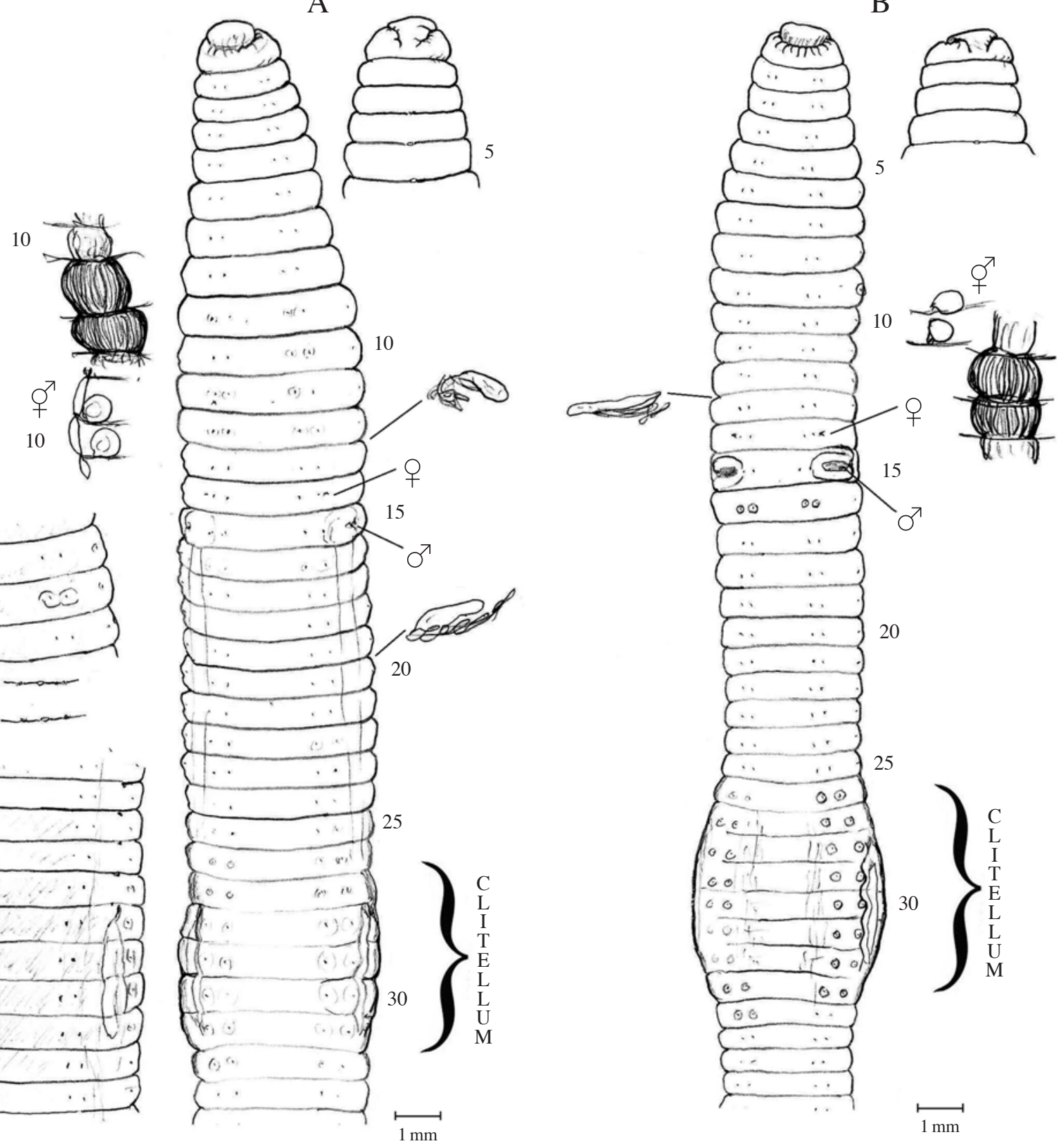

Fig. 16. A Eisenia fetida/andrei specimen providing DNA sample (WM18) ventral view plus prostomium, calciferous glands in 11 \& 12 , some nephridia ( $13 \& 20)$ and the spermathecae in situ in $9 \& 10$; lateral views show pale patch near 9 and tubercula pubertates in 28-31. B Eisenia fetida/andrei specimen providing DNA sample (WO7=w62) for comparison.

\section{?Eisenia andrei Bouché, 1972}

\section{[Fig. 16A]}

Material examined. IV0000249915 mature specimen providing DNA sample (WM18 with nil result, redone as WO12 and as w11 to recheck). From Hamdeok, Sewoobyong beach, Jeju-si, Jocheon-eup, Hamdoek-ri (N33 ${ }^{\circ} 30^{\prime}$ E126 $30^{\prime}$ ) north coast of Jeju-do Island. Collected by R.J. Blakemore, $15^{\text {th }}$ Feb. 2012 from sandy parkland be- hind shops on beachfront, under logs and stones along with many other introduced lumbricids, pheretimoids, and an ocnerodrilid.

Remark. DNA sample WO12 returned a megaBLAST result of $100 \%$ for both E. fetida and for E. andrei (GenBank Nos GU013883.1, FJ214228.1, AY874508.1). Thus $E$. andrei is very tentatively included in the Jeju list. However, there are a dozen species names with priority over the E. andrei associated with E. fetida and no DNA an- 
alysis has yet used types of either taxon to definitively prove their differences. These two species are thus in serious need of revision worldwide. Hong et al. (2001) claimed it from Korean vermiculture based solely on supposed color differences from E. fetida, however E. fetida colour varies greatly and, as noted in its description below, there in no DNA evidence based on types to determine whether on not the name andrei takes priority from the dozen or so synonyms of E. fetida.

\section{Eisenia fetida (Savigny, 1826)} [Fig. 16B]

Material examined. In addition to specimen IV000024 9915 as noted above that provided DNA sample (WO12) and gave a result of either E. fetida or E. andrei. Two other specimens, one IV0000249919 providing DNA sample (WO7 that was mixed, resampled as w62 that is yet to provide results) from Songsaneup, Jeju collected $16^{\text {th }}$ Feb., 2012 by RJB.

Remarks. Cosmopolitan species, this the first record from Jeju; often confused with E. andrei Bouché, 1972 as either a synonym, morph, 'variety', sub-species, or as a separate species; however, several prior synonyms of $E$. fetida compete for priority (Blakemore, 2010b).

\section{Eisenia japonica (Michaelsen, 1892)}

Material examined. Samples from Songsaneup volcanic cone, collected 16 ${ }^{\text {th }}$ Feb. 2012 by RJB (unregistered). Samples from Mt Halla near Nongyo Bridge on Rt 516 $16^{\text {th }}$ Feb., 2012 (unregistered); and further under bridge on Rt 516 two mature specimens, one (IV0000251103) providing DNA (WO45 redone as w18 and as w18b), the other (IV0000251104). Sanbangsan in drain next to Temple, specimens inspected but not registered. DNA results are in the Appendix.

Remarks. This taxon was first recorded on Jeju by Kobayashi (1941) is here confirmed. A redescription based on types by the author is reported in Blakemore and Grygier (2011).

\section{Discussion}

The earliest taxonomic work on Korean earthworms was by Kobayashi $(1934 ; 1936 ; 1938 ; 1941)$, who listed approx. 45 earthworm species from the Korean peninsula (including North and South Korea), of which ca. 27 were considered native (i.e., ca. 18 exotics). He included ten species from Jeju which the current study has raised to about 40 taxa. Surprisingly the two taxa thought most common by Kobayashi, Drawida anchingiana Chen, 1933 and Metaphire quelparta (Kobayashi, 1937), were not relocated possibly because they have been subsumed by newer introductions or because their sub-species were not previously separable without the aid of genetic data. Further surveys are required around Jeju Town, where Kobayashi found some of the Drawida and Metaphire specimens. Newly recognized is the presence of members of the ubiquitous and readily transported Amynthas tokioensis/Metaphire hilgendorfi species-complex but not being as common as on adjacent lands and even on remote Ulleungdo (see Blakemore, 2013).

The conclusion of the relatively high biodiversity is that the interval since introduction of earthworms to this volcanic island, perhaps initially by 'rafting' and more recently by human activity, has since allowed speciation within certain taxa, perhaps related to the selection for the abrasive basaltic soils that Kobayashi (1937) thought hostile to earthworm survival. Although the current species inventory is reasonably high, several other species may be expected with further rigorous study.

The idea that some of the irregular "markings" in Drawida species may actually be parasitic artefacts has yet to be demonstrated, however the appearance of a nematode in the more regular and thus more likely "true" GMs in D. halla is rather unexpected but was not supported by the DNA results. Possibly the DNA primers kits used are more suitable to extract from annelids rather than microbial parasites thus only detecting host tissue.

The possible clonal parthenogentic entities of Amynthas corticis saeseum described in this paper are provided with scientific names, although they may yet prove to be of infra-subspecific rank and thus outside of zoological nomenclature. They are provisionally regarded as a valid taxon and there is slight (1-2\%) mitochondrial DNA detection indicative of divergence. Similarly, there is only slight indication of separation of the A. gracilis insularum sub-species that may be a sexual form and thus more representative of a conventional "species concept" having acquired some genetic modification apparently manifest only on islands. This too has yet to be definitively proven.

Taxonomic revision has obliged several often obvious synonyms to be added to historical taxa and this study has aimed for confidence of combining morphological information with genetic barcoding. Unfortunately there were some initial laboratory errors in failing to get any DNA results, the mixing of samples and contamination in the genetic laboratories/sequencers. Outside of these basic technical problems, the comparisons of molecular data depend entirely upon the reliability of identification of voucher references on GenBank and the Barcode-of-Life library database. Anomalous identifications have been noted in several cases in the current study (e.g. for Metaphire hilgendorfi and Eisenia fetida) as well as current 
lack of comparison for some less common Moniligastridae, Ocnerodrilidae and even Lumbricidae. These are indicative of the relative infancy of this research field that is expected to improve with accumulation of verifiable data with taxonomic names corrected, hopefully based upon their definitive type materials. While the hunt for neotypes of Kobayashi's Jeju worms continues, tangible results can be expected in due course as researchers collaborate to refine use of molecular and computing methods in combination.

\section{ACKNOWLEDGeMENTS}

Collection trips were under the auspices of NIBR with funding from Hanyang University. Research facilites were kindly provided by managers, editors and staff of NIBR, Incheon who also sent off DNA samples for analysis as noted in the Appendix. Referees provided helpful advice although by opting for anonymity the opportunity to openly discuss issues was missed.

\section{REFERENCES}

Blakemore, R.J. 2007. Origin and means of dispersal of cosmopolitan Pontodrilus litoralis (Oligochaeta: Megascolecidae). European Journal of Soil Biology 43 (supplement 1 November, 2007): S3-8 [Available from: http://dx.doi. org/10.1016/j. ejsobi.2007.08.041].

Blakemore, R.J. 2010a. Saga of Herr Hilgendorf's worms. Zoology in the Middle East 49:7-22.

Blakemore, R.J. 2010b. Cosmopolitan Earthworms. $4^{\text {th }}$ Edition. VermEcology So (i)lutions, Yokohama, Japan pp. 750+ 350 Figs. and links.

Blakemore, R.J. 2012a. Amynthas carnosus (Goto \& Hatai, 1899) redescribed on its neotype (Oligochaeta: Megadrilacea: Megascolecidae). Journal of Species Research 1(1): 35-43 [Available from: www.nibr.go.kr/download? attach_path=journal \&attach=2012vol1no1_4.pdf].

Blakemore, R.J. 2012b. On opening a box of worms (Oligochaeta, Megascolecidae) - Historical earthworm specimens transferred to Tokyo from the Saito Ho-on Museum of Natural History in Sendai. Bulletin of the National Science Museum, Tokyo Series A 38(3):95-124.

Blakemore, R.J. 2012c. On Schmarda's lost worm and some newly found New Zealand species. Journal of Species Research 1(2):105-132 [Available from: http://www.nibr. go.kr/english/ event/journal_spe_3.jsp].

Blakemore, R.J. 2012d. Earthworms from NIBR Jeju-do biosphere compared to historical and new Japanese types (Oligochaeta: Megadrilacea: Megascolecidae). Journal of Species Research 1(2):133-115 [Available from: http: //www.nibr.go. kr/english/event/journal_spe_3.jsp].
Blakemore, R.J. 2012e. Restating scope of genus Metaphire Sims \& Easton, 1972: 40 years on (Oligochaeta). Zoology in the Middle East, Supplementum 4:5-14 [Available from: http://www. kasparek-verlag.de/ abstract].

Blakemore, R.J. 2012f. Japanese earthworms revisited a decade on (Oligochaeta: Megadrilacea). Zoology in the Middle East, Supplementum 4:15-22.

Blakemore, R.J. 2013 (current issue). Ullung-do Earthworms - Dagelet Island revisited. Journal of Species Research 2 (1):55-68.

Blakemore, R.J. and M.J. Grygier. 2011. Unravelling some Kinki worms (Annelida: Oligochaeta: Megadrili: Lumbricidae) Part III. Journal of Soil Organisms 83(2):231244.

Blakemore, R.J. and E.K. Kupriyanova. 2010. Unravelling some Kinki worms (Annelida: Oligochaeta: Megadrili: Moniligastridae) Part I. Opuscula Zoologica 40:3-18 [Available from: http://opuscula.elte.hu/PDF/Tomus41_1/ 1_Op\%20-\%20 Blakemore_Drawida.pdf].

Blakemore, R.J., E. Kupriyanova and M.J. Grygier. 2010. Neotypification of Drawida hattamimizu Hatai, 1930 (Oligochaeta: Megadrili: Moniligastridae) and the first COI sequence from an earthworm type. ZooKeys 41:1-29 [Available from: http://pensoftonline.net/zookeys/index. php/journal/article/view/374/401].

Blakemore, R.J., J.-L. Cho and T.-S. Park. 2012. Six exotic terrestrial earthworms (Oligochaeta: Megadrilacea: Moniligastridae, Lumbricidae, Ocnerodrilidae \& Megascolecidae) newly added to Korean species biodiversity list. Zootaxa 3368:300-304 [Available from: http://www. mapress.com/zootaxa /2012/f/zt03368p304.pdf].

Chen, Y. 1933. Preliminary survey of the earthworms of the lower Yangtze Valley. Contributions from the Biological Laboratory of the Science Society of China (Zoology) 9: 177-296.

Gates, G.E. 1935. New earthworms from China, with notes on the synonymy of some Chinese species of Drawida and Pheretima. Smithsonian Miscellaneous Collections 93(7): 1-20 [Available from: http://www.archive.org/stream/ smithsoni anmisce931935smit\#page/n71/mode/1up].

Gates, G.E. 1936. On some species of Chinese earthworms, with special reference to specimens colleced in Szechwan by Dr. D.C. Graham. Proceedings of the United States National Museum 85(3040):405-507 [Available from: http://www. archive.org/stream/proceedingsofuni 851940 unit\#page/406/mode/2up].

Gates, G.E. 1937. Notes on some species of Drawida and Pheretima with description of three new species of Pheretima. Bulletin of the Museum of Comparative Zoology, Harvard 80:305-335.

Gates, G.E. 1972. Burmese Earthworms, an introduction to the systematics and biology of Megadrile oligochaetes with special reference to South-East Asia. Transactions of the American Philosophical Society 62(7):1-326. 
Hong, Y. and S.W. James. 2001a. Five new earthworms of the genus Amynthas Kinberg (Megascolecidae) with four pairs of spermathecae. Zoological Studies 40(4):269-275.

Hong, Y. and S.W. James. 2001b. New species of Korean Amynthas Kinberg 1867 (Oligochaeta, Megascolecidae) with two pairs of spermathecae. Revue Suisse de Zoologie 108(1):65-93.

Hong, Y. and S.W. James. 2009. Some new Megascolecoid earthworms (Oligochaeta). Jounal of Natural History 43: 1229-1256.

Hong, Y. and T.-H. Kim. 2002. Four New Earthworms of the genus Amynthas (Oligochaeta: Megascolecidae) from Korea. Korean Journal of Biological Science 6:195-199.

Hong, Y. and T.-H. Kim. 2005. The earthworm fauna from Ulleungdo Island, Korea with one new specis of the genus Amynthas (Oligochaeta: Megascolecidae). The Korean Journal of Systematic Zoology 21(2):129-136.

Hong, Y. and T.-H. Kim. 2007. Occurrence of earthworm in agro-ecosystem. The Korean Journal of Environmental Biology 25:88-93.

Hong, Y. and T.-H. Kim. 2009. The earthworm composition in agroecosystem of Sunyu Island, Korea. The Korean Journal of Environmental Biology 27(2):135-139.

Hong, Y., T.-H. Kim and Y.-E. Na. 2001. Identity of two earthworms used in vermiculture and vermicomposting in Korea: Eisenia andrei and Perionyx excavatus. The Korean Journal of Systematic Zoology 17(2):185-190.

Kim, T.-H., Y. Hong and N.-J. Choi. 2009. Selection of Earthworms for Bioindicators in Agroecosystems. Korean Journal of Environmental Biology 27(1):40-47.

Kobayashi, S. 1934. Three new Korean earthworms belonging to the genus Pheretima, together with the wider range of the distribution of Pheretima hilgendorfi (Michaelsen). Journal of Chosen Natural History Society 19:1-11.

Kobayashi, S. 1936. Distribution and some external charac- teristics of Pheretima (Ph.) carnosa (Goto et Hatai) from Korea. Science Report of the Tohoku Imperial University 11(1):115-138

Kobayashi, S. 1937. Preliminary survey of the earthworms of Quelpart Island. Science Report of the Tohoku Imperial University (B) 11(3):333-351.

Kobayashi, S. 1938. Earthworms of Korea I. Science Report of the Tohoku Imperial University 13(2):89-170.

Kobayashi, S. 1941. On the terrestrial earthworms from Shikoku, Chieukoku, Kinki and the central region of Japan. Zoological Magazine 53:258-266 (in Japanese).

Shen, H.-P., C.-H. Chang, C.-L. Li, W.-J. Chih and J.-H. Chen. 2013. Four new earthworm species of the genus Amynthas (Oligochaeta: Megascolecidae) from Kinmen, Taiwan. Zootaxa 3599(5):471-482.

Sims, R.W. and E.G. Easton. 1972. A numerical revision of the earthworm genus Pheretima auct. (Megascolecidae: Oligochaeta) with the recognition of new genera and an appendix on the earthworms collected by the Royal Society North Borneo Expedition. Biological Journal of the Linnean Society 4:169-268.

Sims, R.W. and B.M. Gerard. 1999. Earthworms. Synopses of the British Fauna. No. 31 (revised). Field Studies Council, Shrewsbury, pp. 169.

Song, M.J and K.Y. Paik. 1969. Supplemental note of Pheretima kanrazana and Ph. soulensis. Theses Colloquium Communication on $60^{\text {th }}$ Birthday of Dr In Suck Yang, pp. 135-144 [Not currently accessible].

Song, M.-J. and K.-Y. Paik. 1970. Earthworms from Chejoodo Island, Korea. Korean Journal of Zoology 13(1):9-14.

Sun, J., Q. Zhao and J.P. Qiu. 2012. Four new species of the Amynthas corticis-group (Oligochaeta: Megascolecidae) from Hainan Island, China. Zootaxa 2680:149-158.

Submitted: January 21, 2013, Accepted: February 15, 2013 
Appendix - COI barcode preliminary results (in chronological order).

Simply obtaining barcodes has been horrendously difficult and some data are yet wanting. Especially EWU unreliably mixed samples at random. Small tissue from representative specimens, mostly freshly collected, were carefully labelled and refrigerated with batches sent for analysis. Various laboratories were commissioned when initial results were inconsistent. The first "WMxx" series rarely produced data yet used all the DNA extracted requiring resampling from the original specimens as many as five times over three months. The second "WOyy" samples batches either failed to return results or were catastrophically mixed samples in the laboratory invalidating results. Many of the third "wzz" samples returned contamination data due to lack of sequencer hygiene in the NSU. Samples from w43 onwards were performed by different workers outside of NIBR subcontractors, some by Hanyang University student Seunghan Lee, that were mostly successful one year later.

\begin{abstract}
$>$ WM10 Jeju1 \#6 lumbricid tumid 9 (Dendrobeana attemsi?). Species unknown. megaBLAST results $=556 / 658(84 \%)$ for unidentified Amynthas sp. or $<85 \%$ for various megascolecids and only $<81 \%$ from my $D$. attemsi from NZ - thus possibly sample mixed or a new species not yet on GenBank; rechecked as w1 but nil results thus far.

BLAST WM10 vs. WM6 - NZ "Eisenia japonica" Identities=660/660 (100\%) i.e. same! So the NZ sample (Blakemore, 2012c) was contaminated with the Jeju lumbricid sample.

AATCAAAATAGGTGCTGATATAGAATAGGGTCTCCCCCGCCTGCAGGATCGAAGAATGATGTATTTAGGTTTCGGTCTGTTA GAAGTATTGTAATAGCCCCAGCCAGTACGGGCAATGAGAGCAATAGAAGTACTACGGTAATAACCACTGCTCATACAAATA GGGGAATTCGTTCTAATCGTAGTCCCGATCAGCGTATATTAATTACTGTGGTGATGAAGTTGATAGCCCCTAGAATTGAGGA TGCTCCGGCTAAGTGGAGTGAGAAAATTGCTAGATCCACAGAAGGTCCAGCATGTGCAATATTTCTTGCTAGGGGTGGATAT ACCGTTCACCCGGTTCCTGCCCCCTTCTCTACTGCTGCTGAGGATACTAGTAAAATTAGAGACGGCGGTAGTAGTCAGAATC TTATGTTATTTAGTCGTGGGAATGCTATGTCTGGTGTCCCCAATATAAGAGGTAGTAGCCAATTTCCAAAGCCCCCAATAAA TACTGGTATAACTAAGAAAAAGATTATTAGAAATGCGTGTGCAGTAACAATTGTGTTGTATAGTTGATCGCTCCCTATAAAG GACCCGGGCTGCCTTAGCTCAATCCGAATAAGTAATCTTATCCCGGCACCCACCATGCCGGCCCAAATTCCTAAGATAAAAT ATAAAGTTCCA
\end{abstract}

\begin{abstract}
$>$ WM11 Jeju1 \#3 Amynthas arx H from beach at "Sunrise Peak".
megaBLAST nothing better than $<86 \%$ for M. sieboldi, M. tschiliensis or A. vittatus - i.e., a new species or not yet on GenBank; rechecked as $\mathrm{W} 4$.

TAGGTGTTGGTAGAGGATTGGATCCCCTCCCCCGGCCGGGTCGAAGAAGGATGTATTTAGATTTCGATCTGTTAATAGTATT GTAATGGCTCCCGCTAGCACAGGTAGGGATAGGAGTAGTAGTACCACAGTAATTACGACTGCCCAGACAAATAGTGGGATT CGCTCTAATCGTAAACCTGATCAACGTATATTAATTACAGTGGTGATAAAGTTGATCGCCCCCAGAATTGATGATGCCCCCG CCAAGTGAAGGGAGAAAATTGCAAGATCTACAGATGGCCCAGCATGTGCAATATTTCTTGCAAGGGGTGGATATACTGTTC ATCCTGTCCCTGCCCCTTTTTCAACGGCAGCAGATGATACTAATAAAATAAGTGATGGGGGGAGTAATCAAAATCTCATATT ATTTAGTCGTGGGAATGCTATATCTGGTGTCCCCAGTATTAGGGGTAAAAGTCAGTTTCCAAATCCCCCAATAAATACTGGT ATTACTAGAAAGAAAATTATTAAAAATGCGTGTGCTGTAACAATAGTATTATAGAGTTGGTCTCTACCTAGGAATGATCCTG GTTGTCTTAGCTCAACTCGAATAAGGAGTCTCATACCTGCTCCAATTATCCCAGCTCAAATACCTAAAATGAAATAAA
\end{abstract}

\begin{abstract}
> WM12 Jeju1 \#5 Metaphire haenyeo H sp. nov from Machair(redone as w5).
megaBLAST result 93\% for "Lumbricina sp. - Chinese Herbal Medicine"; or <87\% A. fuscatus from Japan, or A. tuberculatus/M. tschiliensis from China.

WM12 vs. w20 M. q. valhalla Identities=650/657 (98.9\%) i.e., similar/same samples $/$ species.

AATAGGTGTTGATATAGAATTGGATCTCCTCCACCAGCCGGATCAAAAAATGATGTATTTAGGTTTCGATCTGTTAATAACA TTGTAATAGCCCCTGCTAGTACCGGTAGGGATAGTAGTAGTAATACTACGGTAATTACTACTGCTCACACAAATAGTGGGAT TCGCTCTAACCGCAGTCCCGATCACCGCATATTAATTACAGTAGTGATGAAATTAATAGCTCCCAAAATTGATGATGCCCCA GCAAGATGAAGTGAAAAGATTGCCAAATCTACTGAAGGTCCAGCGTGGGCAATATTTCTTGCTAGTGGGGGATATACTGTT CACCCTGTTCCTGCACCCTTTTCAACTGCCGCGGATGATACTAGTAGAATAAGTGAGGGTGGTAGAAGTCAGAAACTTATAT TATTTAGTCGTGGGAAAGCTATGTCTGGTGTCCCAAGTATTAGGGGGAGTAGCCAGTTTCCGAAGCCCCCAATAAATACTGG TATTACCAGAAAGAAAATTATTACGAATGCGTGAGCAGTTACAATTGTATTATATAGTTGATCTCTTCCTAGAAATGATCCA GGCTGTCTTAGCTCAATTCGAATAAGTAGTCTTATACCGGCTCCAATTATTCCAGCTCAAATACCCAAAATAAAATATAGTG TT
\end{abstract}

> WM13 Jeju1 \#8 Sanbansan Temple big green damaged Metaphire - BLAST ditto WM12 “chinese herbal medicine” and WO11 redo of
WM16! But WM13 vs. WM12 Identities=631/650 (97\%); and WM13 vs. WO11=646/646(100\%)! So WM13=WO11.
AGAATAGGTGTTGATATAGAATTGGATCTCCTCCACCAGCTGGATCAAAGAATGATGTATTTAGGTTCGATCTGTTAATAA
TATTGTAATAGCCCCTGCTAGTACCGGTAGGGATAGTAGTAGTAATACTACGGTAATTACTACTGCTCACACAAATAGGGGG
ATTCGCTCTAACCGCAGTCCCGATCACCGCATATTAATTACAGTAGTGATGAAATTAATAGCTCCCAAAATGATGATGCCC
CAGCAAGATGAAGTGAAAAGATTGCCAGGTCTACTGAAGGTCCAGCGTGGGCAATATTTCTGCTAGTGGGGGATATACTG
TCCACCCTGTTCCTGCACCTTTTCAACTGCGGCGGATGACACCAATAGAATAAGTGAGGGTGGCAGAAGTCAGAATCTTAT
ATTATTTAGTCGTGGGAAAGCTATGTCTGGTGTCCCGAGTATTAGGGGGAGTAGCCAGTTTCCGAACCCCCAATAAATACT
GGTATTACCAGAAAGAAAATTATTACGAATGCGTGAGCAGTTACAATTGTATTATATAGTTGATCTCTTCCTAGAAATGATC
CAGGTTGTCTTAGCTCAATTCGAATAAGTAATCTTATACCGGCTCCAATTATTCCGGCTCAAATACCCAAAATAAAATA 
$>$ WM14 Korea $1^{\text {st }}$ survey from smallest lumbricid - BLAST 84\% Dendrodrilus rubidus from Austria, i.e. different species not yet on GenBank.

AGTGTTGATATAAAATTGGATCTCCTCCCCCCGCAGGGTCAAAAAATGAAGTGTTTAAGTTTCGATCAGTTAATAATATAGT AATTGCGCCAGCAAGAACTGGAAGAGATAAAAGAAGTAAAACAACAGTAATTAGAACTGCTCATACAAATAAGGGAATCC GTTCTAAACGTAACCCGGCTCATCGTATGTTAATGACGGTGGTGATAAAGTTAATTGCACCAAGAATTGAAGATGCACCTGC CAGGTGAAGGGAGAAAATTGCTAGATCTACTGAAGGTCCAGCATGAGCAATATTTCTAGAGAGAGGAGGGTATACAGTTCA ACCAGTTCCAGCTCCTTTTTCTACTGCCGCAGAGGATACTAGGAGAATCAGTGAAGGAGGTAAAAGTCAGAATCTTATATTA TTTAACCGAGGAAATGCTATATCTGGTGCACCTAGTATTAATGGAAGAAGTCAATTTCCAAATCCTCCAATAAATACAGGCA TAACTAGGAAGAAAATTATTACGAAGGCATGGGCTGTAACGATTGTATTATATAATTGATCCCTTCCTAAGAAAGCTCCAGG TTGTCTTAATTCAATTCGAATTAGAAGTCTTATTCCGGCACCTACTATTCCGGCTCAGACACCTAAAATAAAGTAAA

$>$ WM17 Jeju1 \#1 Oc. occidentalis tissue - megaBLAST nothing better than < 85\% for random megadriles, i.e., nothing similar yet available on GenBank/iBOLD.

GGTGTTGATATAAAATTGGGTCTCCTCCTCCTGCTGGATCAAAGAATGAGGTATTTAGATTTCGATCTGTTAGGAGTATTGTA ATAGCTCCTGCTAGTACAGGAAGTGATAATAGTAGAAGTACTACCGTAATTACTACTGCTCATACAAATAATGAGATTCGTT CTAATCGTAGGCCATTTCATCGTATATTAACTACTGTAGTGATAAAGTTGATTGCCCCTAAAATTGATGAGGCACCGGCTAG ATGAAGTGAGAAAATTGCTAGGTCTACTGATGGTCCTGCGTGTGCTATATTTCTGGCTAGTGGTGGATATACTGTTCATCCA GTTCCTGCTCCTTTTTCTACTGCTGCGGATGAGACTAGTAGGATTAGGGCAGGAGGGAGTAGTCAGAATCTTATGTTATTTA GTCGTGGGAAGGCTATATCTGGTGCGCCTAGTATTAAAGGTAGTAATCAGTTTCCAAACCCCCCAATAAATACTGGTATTAC CAGGAAGAAAATTATTAAAAAGGCATGTGCTGTTACGATAGTATTATATAGTTGATCTCTGCCTAGGAATGCACCTGGTTGC CTTAATTCAATTCGAATTAATAGTCTTATTCCGGCCCCTACTATTCCTGCTCATACCCCTAGGATAAAATATAAGGT

> WM18 Jeju1 \#1 Red dorsum Eisenia worm NIL result - redone as WO12

> WO7 Jeju1 \#4 Pale lumbricid (Eisenia fetida?)dissected on sheet 26.III.2012 - BLAST A. trapezoides 100\%. (Same 100\% as WO13 - i.e., mixed sample or contamination?)

AACCCTATATTTTATTCTTGGGGTCTGAGCTGGCATAGTGGGTGCCGGTATAAGGCTCCTTATTCGAATTGAGCTAAGTCAG CCGGGAGCTTTCCTAGGAAGAGACCAACTCTACAATACCATTGTTACAGCCCATGCATTTGTAATAATTTTCTTTTTAGTTAT GCCCGTATTTATTGGTGGCTTCGGAAACTGACTACTACCCCTAATACTAGGAGCTCCAGATATAGCCTTTCCTCGACTAAAT AACATAAGATTCTGGCTACTACCACCCTCATTAATTCTTCTAGTATCTTCTGCTGCTGTAGAAAAAGGAGCAGGAACAGGTT GAACAGTATATCCACCATTAGCCAGAAATCTAGCCCATGCCGGCCCATCAGTAGACCTTGCCATTTTCTCACTTCATTTAGC AGGGGCATCTTCAATTCTAGGAGCTATCAACTTTATTACAACCGTAATCAACATACGGTGAAGTGGTTTACGACTAGAACGA ATCCCCTTATTTGTATGGGCAGTAGTAATTACTGTTGTGCTTCTTCTTATATCCCTACCAGTGCTTGCAGGAGCTATTACTATG CTTTTAACAGACCGAAATCTTAACACTTCATTCTTTGACCCTGCCGGAGGAGGAGACCCTATTTTATATCAACATCTATT

$>$ WO11 Jeju1 \#8 Big damaged pale Amynthas (redo of WM16) - ditto "chinese herbal medicine" and WO11 vs WM13 Identities= 646/646 (100\%), Gaps $=0 / 646(0 \%)$ ! i.e. WM13=WO11.

GGTGTTGATATAGAATTGGATCTCCTCCACCAGCTGGATCAAAGAATGATGTATTTAGGTTTCGATCTGTTAATAATATTGTA ATAGCCCCTGCTAGTACCGGTAGGGATAGTAGTAGTAATACTACGGTAATTACTACTGCTCACACAAATAGGGGGATTCGCT CTAACCGCAGTCCCGATCACCGCATATTAATTACAGTAGTGATGAAATTAATAGCTCCCAAAATTGATGATGCCCCAGCAAG ATGAAGTGAAAAGATTGCCAGGTCTACTGAAGGTCCAGCGTGGGCAATATTTCTTGCTAGTGGGGGATATACTGTCCACCCT GTTCCTGCACCTTTTTCAACTGCGGCGGATGACACCAATAGAATAAGTGAGGGTGGCAGAAGTCAGAATCTTATATTATTTA GTCGTGGGAAAGCTATGTCTGGTGTCCCGAGTATTAGGGGGAGTAGCCAGTTTCCGAAACCCCCAATAAATACTGGTATTAC CAGAAAGAAAATTATTACGAATGCGTGAGCAGTTACAATTGTATTATATAGTTGATCTCTTCCTAGAAATGATCCAGGTTGT CTTAGCTCAATTCGAATAAGTAATCTTATACCGGCTCCAATTATTCCGGCTCAAATACCCAAAATAAAATATAGTGTT

\begin{abstract}
> WO12 Jeju1 \#1 Red backed Eisenia lumbricid (redo of WM18) BLAST - 100\% for "DNA barcodes for soil animal taxonomy: transcending the final frontier" by Bouche and James from France as "Lumbricidae sp." or 100\% E. fetida or 100\% E. andrei; GenBank Nos GU013883.1, FJ214228.1, AY874508.1. October redo W11 exactly same results as WO12.

ATAAATGTTGGTAGAGAATAGGGTCGCCACCTCCAGCAGGGTCAAAGAATGAGGTATTTAGGTTTCGATCTGTCAATAGTAT AGTGATAGCTCCCGCAAGTACTGGAAGAGATAAAAGTAGTAACACCACGGTAATAACTACAGCTCATACAAATAGGGGGAT TCGTTCTAGTCGAAGCCCACTTCATCGTATGTTAATAACTGTAGTAATGAAGTTAATTGCCCCTAAAATTGAGGAGGCACCT GCTAAATGGAGGGAAAAAATAGCCAGGTCCACTGAGGGCCCCGCGTGCGCTAAGTTACTGGATAGGGGTGGGTAAACTGTC CACCCTGTTCCAGCACCCTTTTCCACTGCAGCAGAGGATACTAGGAGAATTAGGGAAGGGGGCAGAAGTCAAAATCTTATG TTGTTGAGACGTGGAAAGGCTATGTCTGGAGCTCCTAGTATAAGAGGTAGAAGTCAGTTTCCAAATCCACCAATAAATACA GGTATTACCAGAAAGAAAATTATTACAAATGCATGGGCTGTAACAATTGTATTATATAGTTGGTCCCTTCCTAGGAAGGCAC CTGGTTGCCTTAGCTCGATTCGAATGAGAAGGCTTATACCAGCACCAACCATACCTGCTCAGACCCCGAGAATGAAATAGA G
\end{abstract}

> WO13 Jeju1 \#1 smaller lumbricids in vial B. parvus - megaBLAST A. trapezoides 100\% (same as WO7! - contamination or mixed in lab.?)

TGGAACCCTATATTTTATTCTTGGGGTCTGAGCTGGCATAGTGGGTGCCGGTATAAGGCTCCTTATTCGAATTGAGCTAAGTC AGCCGGGAGCTTTCCTAGGAAGAGACCAACTCTACAATACCATTGTTACAGCCCATGCATTTGTAATAATTTTCTTTTTAGTT 
ATGCCCGTATTTATTGGTGGCTTCGGAAACTGACTACTACCCCTAATACTAGGAGCTCCAGATATAGCCTTTCCTCGACTAA ATAACATAAGATTCTGGCTACTACCACCCTCATTAATTCTTCTAGTATCTTCTGCTGCTGTAGAAAAAGGAGCAGGAACAGG TTGAACAGTATATCCACCATTAGCCAGAAATCTAGCCCATGCCGGCCCATCAGTAGACCTTGCCATTTTCTCACTTCATTTAG CAGGGGCATCTTCAATTCTAGGAGCTATCAACTTTATTACAACCGTAATCAACATACGGTGAAGTGGTTTACGACTAGAACG AATCCCCTTATTTGTATGGGCAGTAGTAATTACTGTTGTGCTTCTTCTTATATCCCTACCAGTGCTTGCAGGAGCTATTACTAT GCTTTTAACAGACCGAAATCTTAACACTTCATTCTTTGACCCTGCCGGAGGAGGAGACCCTATTTTATATCAACATCTATT

\begin{abstract}
$>$ WO16 Jeju1 \#1 dark A. trapezoides not figured-megaBLAST only <84\% Dendrodrilus rubidus. i.e. new or more likely mixed in the laboratory! CTTTACTTTATTTTAGGTGTCTGAGCCGGAATAGTAGGTGCCGGAATAAGACTTCTAATTCGAATTGAATTAAGACAACCTG GAGCTTTCCTAGGAAGAGATCAATTATATAATACAATTGTTACAGCCCACGCCTTCGTAATAATTTTCTTCCTAGTTATGCCT GTATTTATTGGAGGATTTGGAAATTGACTTCTTCCATTAATACTAGGTGCACCAGACATAGCATTTCCTCGATTAAATAATAT AAGATTCTGACTTTTACCTCCTTCACTGATTTTCTTAGTTTCTTTTGCGGCAGTAGAAAAAGGAGCGGGAACTGGTTGAACGG TATACCCTCCTCTCTCTAGAAATATTGCTCATGCTGGACCTTCAGTAGATCTAGCAATTTTCTCCCTTCACCTGGCAGGTGCA TCTTCAATTCTTGGTGCAATTAACTTTATCACCACTGTCATTAACATACGATGAGCCGGGTTACGTTTAGAACGGATTCCCTT ATTTGTATGAGCAGTTCTAATTACTGTTGTTTTACTTCTTTTATCTCTTCCAGTTCTTGCTGGCGCATTTACTATATTATTAACT GATCGAAACTTAAACACTTCATTTTTTGACCCTGCGGGAGGGAGGAGATCCAATTTTATATCAAC
\end{abstract}

\begin{abstract}
> WO17 Jeju1 \#1 pale A. caliginosa not figured - - megaBLAST nothing better than 83\% lumbricids (new?) ? redone below as wo17b. BLAST WO17 vs wo17b Identities $=480 / 636(75 \%)$ ! Thus samples were mixed in lab.

ACTCTATATTTTATTCTAGGAGTATGAGCCGGCATAGTAGGTGCTGGAATAAGTCTTCTTATTCGAATTGAGCTAAGTCAAC CTGGGGCTTTTCTAGGTAGAGATCAACTCTACAACACGATTGTTACAGCTCATGCATTTGTGATAATCTTCTTCTTAGTAATA CCCGTATTTATTGGAGGATTTGGAAACTGACTCTTACCTCTAATATTAGGAGCACCTGATATAGCCTTCCCCCGTCTAAATAA TATAAGATTCTGATTACTACCTCCTTCCCTCATTCTTCTTGTATCTTCTGCAGCAGTAGAAAAAGGGGCCGGAACAGGCTGA ACCGTATATCCCCCTCTATCGAGGAACCTTGCACATGCCGGCCCATCCGTAGATTTAGCTATCTTCTCCCTTCATCTAGCAGG AGCTTCTTCTATTCTCGGGGCCATCAATTTCATTACTACAGTTATTAATATGCGGTGAACAGGAATACGTCTAGAACGAATC CCTTTATTTGTTTGAGCTGTAATCATTACAGTAGTTCTTCTTCTTCTTTCACTTCCAGTGCTTGCTGGAGCAATTACAATGCTC CTAACAGATCGAAACCTCAATACTTCATTCTTTGATCCTGCGGGAGGAGGAGACCCTATCCTTTATCAACATCTATTC
\end{abstract}

\begin{abstract}
$>$ WO17b redo of Jeju \#1 pale A. caliginosa - megaBLAST=99\% "Drawida sp. Watarase" i.e. mixed sample again! - maybe first WO17a is correct. But what is this? Data is the same as WO28 D. koreana from Shindo and with WO32a "P. sanctaehelenae" types! I.e., earthworm sample of unknown origin mixed in genetics lab.

TTTATTTTAGGTGTATGAGCTGGGATAATTGGTGCGGGAATGAGGCTATTAATTCGAATTGAGTTAAGACAACCAGGTTCGT TTTTAGGCAGAGATCAGCTTTATAATACTATTGTTACTGCACATGCTTTTATTATAATTTTTTTCTTAGTAATACCCGTATTTA TTGGTGGCTTTGGTAATTGATTATTACCCTTAATACTTGGGGCTCCAGACATGGCTTTCCCTCGACTTAATAATTTAAGATTT TGGCTCTTACCGCCGGCTTTAATTCTTTTAGTGTCGTCAGCAGCAGTGGAAAAGGGTGCTGGTACCGGGTGAACAGTTTATC CGCCTCTGGCTAGTAATTTAGCACATGCGGGCCCATCAGTCGACCTAGCGATTTTTTCTTTACATTTGGCAGGAGCATCCTCT ATTTTAGGGGCCACTAATTTTATTACAACTGTTATTAACATACGATGGGTTGGCATACACTTTGAGCGAATTCCGCTATTCGT GTGGGGAGTTTTAATTACTGTAGTACTATTACTTCTATCTTTGCCTGTGTTGGCCGGCGCAATTACTATGCTTTTAACCGATC GAAACTTAAATACTTCATTCTTTGATCCGGCCGGAGGCGGAGACCCTATTTTATACCAGCATT
\end{abstract}

\begin{abstract}
$>$ WO35 Nogeyama A masatakae 5/5/12 - megaBLAST=99-100\% “A. triastrianus" from China or “A. robustus" from Japan - these misidentifications, as expected!

WO35 vs. w28b Identities $=657 / 657(100 \%)$, i.e. A. masatakae confirmed from Jeju. QED.

WO35 vs. w29 Identities $=614 / 657(93 \%)$, i.e. different taxa (=A. tralfamadore).

WO35 vs. w30 Identities $=617 / 657(94 \%)$, i.e. different taxa $(=A$. tralfamadore $)$.

GGAACCTTATACTTTATCCTGGGAATTTGAGCAGGAATAATTGGTGCAGGAATAAGACTACTTATTCGTATTGAATTAAGAC AACCGGGATCCTTCCTTGGTAGAGACCAACTATATAATACAATTGTAACAGCACATGCATTCTTAATAATCTTCTTTCTAGTA ATGCCAGTATTTATTGGTGGATTCGGAAACTGGCTACTGCCCCTAATACTTGGAACCCCAGATATGGCGTTCCCCCGATTAA ATAACATAAGATTTTGACTACTTCCACCATCCCTAATTTTATTAGTAAGATCGGCCGCGGTAGAAAAAGGAGCGGGCACAG GTTGGACAGTTTACCCACCCCTAGCAAGAAATATAGCACATGCCGGCCCTTCAGTAGACCTTGCAATTTTCTCACTCCATCTT GCAGGTGCCTCATCAATTCTTGGAGCTATTAACTTTATCACAACAGTAATTAACATGCGATGATCTGGGCTACGTCTAGAAC GAATCCCACTATTTGTATGAGCAGTAGTGATCACCGTAGTACTATTATTATTATCCCTACCTGTACTGGCTGGGGCAATTACC ATATTACTAACGGATCGAAACCTTAATACATCATTCTTTGACCCAGCAGGAGGGGGGGACCCAGTTCTATACCAACACCTAT
\end{abstract} $\mathrm{T}$

$>$ WO38 April $18^{\text {th }}$ Tubificidae - megaBLAST 99\% Pontodrilus litoralis - i.e., sample mixed probably with WO39 that is $P$. litoralis TACTTCATCCTAGGAGTATGAGCTGGGATAATTGGAGCTGGAATAAGACTACTTATTCGAATTGAACTAAGACAACCAGGA GCATTTCTAGGGAGAGATCAACTATATAATACAATTGTTACTGCACACGCATTTCTAATAATTTTTTTCTTAGTAATACCTGT ATTTATTGGAGGATTTGGAAACTGATTACTACCCCTTATACTAGGAGCACCAGATATAGCTTTTCCACGACTAAACAACATA AGATTTTGACTTCTACCTCCTTCTCTAATCCTTCTAGTTTCATCAGCTGCAGTAGAAAAAGGTGCAGGAACTGGATGAACAG TTTATCCACCATTAGCAAGTAATATTGCCCATGCTGGGCCATCAGTAGATCTAGCCATTTTTTCACTACATTTAGCCGGGGCT TCATCAATTCTAGGAGCTATTAACTTTATTACAACAGTTATTAATATACGATGATCAGGATTACGACTAGAACGAATTCCAT 
TATTCGTATGGGCTGTAGTTATTACAGTAGTGCTACTACTATTATCCCTTCCTGTGTTAGCAGGGGCTATTACAATACTATTA ACAGATCGCAATCTAAATACATCTTTCTTCGACCCAGCAGGTGGGGGAGATCCA

\footnotetext{
> WO39 - Pontodrilus litoralis - nil result (but cf. WO38!).

$>$ WO40 - Metaphire q. valhalla - nil result (redone as w20).

$>$ WO41 - Amynthas aucklandis - nil result (redone as w4).

$>$ WO42 - Metaphire cf. phaselus - nil result (redone as w22).

$>$ WO43 - Amynthas simplex $\mathrm{H}$ - nil result (redone as w23).
}

> WO44 "Drawida cf. koreana" Jeju \#2 June 2012 - megaBLAST 94\% some lumbricids, i.e., mixed in lab with "Eisenia cf. japonica" sample (WO45) as this barcode data is the same as for w18b redone for Eisenia japonica from Jeju (cf. WO45). WO44 redone as w17.

$>$ WO45 "Eisenia cf. japonica from Mt Halla Jeju \#2" megaBLAST $=<82 \%$ for some Moniligastrid, i.e., sample mixed in lab. BLAST WO45 vs. w24 Identities $=650 / 650(100 \%)$. [WO45 vs. WO28 Identities $=516 / 648(80 \%)$, i.e., "D. cf. koreana" from Incheon is a different species]. Sample "WO45" was mixed in the genetics lab probably with WO44. WO45 redone as w18.

TTATATTTCATTTTAGGTGTGTGGGCAGGTATAATTGGTGCTGGTATAAGTCTTTTGATTCGGATTGAATTAAGACAGCCTGG CTCATTTTTGGGAAGCGATCAGCTCTATAATACTATTGTTACTGCTCATGCTTTCGTTATAATTTTTTTTCTTGTAATACCTGT ATTTATTGGTGGCTTCGGAAATTGACTTCTTCCTCTTATACTAGGGGCTCCGGATATAGCATTCCCACGTCTTAATAATCTGA GATTTTGGCTATTGCCCCCAGCATTAATTCTTTTAGTTTCTTCGGCAGCAGTGGAAAAAGGTGCTGGTACTGGGTGAACAGT ATACCCACCGCTAGCAAGCAACCTGGCTCACGCCGGTCCCTCAGTTGATTTAGCAATTTTTTCTCTTCATTTAGCAGGAGCGT CTTCAATTTTGGGCTCAATTAATTTTATTACAACTGTAATTAATATGCGATGATCTGGGATAAATGTAGAGCGAATTCCACTA TTTGTTTGAGGTGTAGTTATTACTGTAGTATTACTTTTATTATCTCTTCCGGTTTTAGCGGGAGCAATTACTATGCTTTTGACA GATCGAAATCTGAATACGTCTTTCTTCGATCCTGCCGGTGGTGGAGACCCAATTCTGTATCAGCACTT

> WO46 Drawida sp Jeju \#2 June, 2012. mega BLAST=Amynthas corticis 100\%, i.e., (apparently mixed in lab with sample " $>$ WO47 of Amynthas corticis". Resampled as w24.

$>$ WO47 - A. corticis Hyomyungsa, Halla $12^{\text {th }}$ June, 2012 - nil result (resampled as w25).

$>$ WO48 - A. micronarius from Hyomyungsa Temple, Jeju $12^{\text {th }}$ June, 2012 - nil result.

$>$ WO49 Amynthas sp. \#1 immature from Hyonyungsa Temple, Hallasan, Jeju $12^{\text {th }}$ June, $2012-$ megaBLAST $=100 \%$ M. hilgendorfi (AB543234) and 100\% A. tokioensis (AB542560, 542556) from Japan which are either (or both!) misidentifications. It is not known if this sample is reliable or mixed but it provides evidence of the M. hilgendorfi spp-gp. on Jeju.

ACTATACTTCATTCTAGGTATTTGAGCTGGAATAATTGGAGCAGGAATAAGACTCCTTATTCGAATTGAGCTAAGACAAGCC GGGTCCTTTTTAGGTAGAGACCAACTCTACAATACAATCGTAACAGCTCATGCATTCTTAATAATTTTCTTTCTTGTAATGCC TGTATTTATTGGAGGATTTGGAAATTGATTACTACCTCTAATGTTAGGTGCACCCGATATAGCATTTCCACGACTCAACAATA TAAGATTTTGATTACTGCCCCCGTCACTAATTCTACTAGTATCCTCGGCTGCGGTAGAGAAAGGTGCAGGTACAGGATGAAC AGTATATCCCCCACTATCAAGAAATATTGCTCATGCAGGCCCATCAGTAGATCTAGCTATTTTCTCACTCCACTTAGCAGGTG CCTCTTCAATTTTAGGTGCTATCAACTTTATTACCACAGTAATTAATATACGATGATCCGGACTACGCTTAGAGCGAGTACCT CTATTTGTATGAGCAGTTGTAATTACTGTAGTTCTTCTACTTCTATCTCTGCCAGTCCTAGCAGGTGCTATCACAATACTACT AACAGATCGAAATCTAAATACATCTTTTTTTGATCCAGCAGGTGGGGGAGACCCAATTCTATATCAACACCTAT

$>$ WO50 A. corticis \#2 imTemple Jeju ? megaBLAST =A. corticis $90 \%$ - i.e., mixed in lab with WO47 or WO51?

CTCTATATTTCATTTTAGGAATTTGAGCCGGAATAATTGGAGCAGGGATAAGCCTACTTATCCGAATTGAATTAAGACAACC AGGATCATTCCTTGGAAGTGATCAACTATATAACACAATTGTAACAGCACATGCATTCTTAATAATCTTTTTTCTAGNGATAC CAGTATTTATTGGTGGATTTGGAAACTGATTATTACCCCTTATATTAGGGACTCCAGACATAGCATTCCCTCGACTAAACAAT ATGAGATTTTGACTATTGCCACCCTCACTGATCTTACTAGTCTCATCTGCCGCAGTAGAAAAAGGTGCAGGGACAGGGTGGA CAGTTTACCCACCCCTAGCAAGAAATATCGCACATGCGGGACCATCAGTAGATCTTGCAATTTTCTCACTACACTTAGCAGG AGCATCATCAATCTTGGGAGCAATTAATTTTATTACCACAGTAATTAATATACGGTGATCTGGGCTACGACTAGAACGAATT CCACTATTTGTCTGAGCAGTGGTAATTACCGTAGTTTTACTACTTTTATCACTACCAGTACTAGCCGGAGCTATTACAATACT ACTAACAGATCGAAACCTTAATACATCATTCTTTGACCCAGCAGGGGGAGGAGACCCAATTTTATATCAACACCTA

\footnotetext{
$>$ WO51 - "A. gracilis?" from Cheonji Falls - nil result. Redone as w27.

$>$ WO52 - A. tralfamadore/masatakae from Cheyonji Falls - nil result. Redone as w28a, b.

$>$ WO53 - darker A. tralfamadore/masatakae from Cheyonji Falls - nil result. Redone as w29.
}

$>$ WO54 paler A. tralfamadore/masatakae from Cheyonji. Seogwipo Falls paler spec-Amynthas gracilis $100 \%$ - I need to CHECK … most likely mixed with WO55? - redone as w30 (QED).

CCCTATATTTTATTCTAGGAATTTGAGCCGGAATAATTGGAGCTGGAATAAGGCTACTTATTCGAATTGAACTCAGACAGCC GGGATCGTTTCTGGGAAGAGATCAACTATATAATACAATTGTAACAGCTCATGCATTTGTAATAATTTTCTTTCTGGTAATAC CAGTATTCATTGGTGGATTTGGAAACTGACTACTACCTCTAATGCTGGGTACACCAGACATAGCATTTCCGCGACTTAATAA 
TATAAGATTTTGACTACTCCCCCCGTCACTTATCTTACTAGTAAGATCCGCGGCCGTTGAAAAGGGGGCGGGAACTGGATGA ACAGTATATCCCCCGCTGGCAAGAAATATTGCACATGCTGGTCCATCAGTAGATCTAGCAATCTTCTCACTACACTTGGCAG GAGCATCATCTATTCTTGGGGCCATTAACTTTATTACAACTGTAATTAATATACGATGATCTGGATTACGGCTAGAACGAAT CCCCCTATTTGTATGGGCCGTAGTAATTACTGTAGTACTTCTACTATTATCTCTACCTGTACTAGCCGGAGCTATTACTATATT ATTAACAGATCGAAACCTTAACACATCATTCTTTGATCCCGCTGGAGGTGGAGACCCTATTCTATATCAACACCTA

$>$ WO55 - Amynthas sp. Cheyonji - nil result. Redone w31.

$>$ WO56 Metaphire $\mathrm{cf}$ agrestis from Cheonji - megaBLAST $=100 \%$ A. corticis - i.e., WO56 was mixed in lab (with WO57). BLAST WO56 vs. w33 Identities $=646 / 646(100 \%)$ i.e., confirming samples were mixed.

$>$ WO57 A. corticis saeseum-megaBLAST M. californica <99\%, i.e WO57 mixed in lab (with WO58)? Redone as w33.

CCTTATATTTTATTCTAGGAATCTGAGCAGGGATAATCGGAGCAGGGATAAGATTACTTATTCGCATCGAACTAAGACAGCC TGGATCATTCCTTGGAAGAGACCAACTATATAATACAATTGTGACAGCACACGCATTTNTAATAATTTTCTTTNTGGTGATA CCAGTATTTATTGGGGGATTTGGAAACTGACTTCTCCCACTAATGTTAGGAACCCCTGACATAGCGTTCCCTCGACTAAATA ACATAAGATTCTGACTACTGCCACCCTCATTAATTCTACTAGTTAGATCCGCGGCAGTAGAAAAGGGAGCAGGTACAGGAT GAACAGTGTACCCTCCACTAGCTAGAAACATAGCACACGCAGGTCCATCAGTAGATCTTGCAATTTTCTCACTACATTTAGC AGGTGCCTCATCAATTTTGGGGGCCATCAATTTCATTACCACTGTGATCAACATGCGATGATCAGGCCTACGCCTAGAGCGA ATCCCACTATTCGTATGAGCCGTAGTAATCACTGTAGTACTCCTTCTACTATCACTTCCTGTGCTAGCGGGAGCAATTACTAT ATTATTAACGGATCGAAACCTAAATACCTCATTCTTCGACCCTGCA

> WO58 - M. californica from Saeseum Island - nil results (cf. WO57). Resampled as w34.

> WO66 Green M. quelparta seogwipo from Jeju bot gars 9/9/12 - megaBLAST < 93\% for "Chinese herbal medicine" species. BLAST WO66 vs. WM11 Identities=550/648 (84.9\%) shows Amynthas arx to be a different taxon while WO66 vs. WM12 Identities=635/657 $(96.7 \%)$, WO66 vs. WM13 Identities $=633 / 652(97.1 \%)$ and WO66 vs. w20 M. q. valhalla Identities $=637 / 658(96.8 \%)$, ? i.e., possibly subspecies?

GGTCAACAAATCATAAAGATATTGGAACACTATATTTTATCTTGGGTATTTGAGCCGGAATAATTGGAGCCGGTATAAGATT ACTTATTCGAATTGAGCTAAGACAACCTGGATCATTTCTAGGAAGAGATCAACTATATAATACAATTGTAACTGCTCACGCA TTCGTAATAATTTTCTTTCTGGTAATACCAGTATTTATTGGGGGTTTCGGAAACTGGCTACTCCCCCTAATACTTGGGACACC AGACATAGCTTTCCCACGACTAAATAATATAAGATTTTGACTTCTACCCCCCTCACTTATTCTATTAGTGTCATCCGCCGCAG TTGAAAAGGGTGCAGGGACAGGATGAACAGTATATCCCCCACTAGCAAGAAATATTGCCCACGCTGGACCTTCAGTAGATC TGGCAATCTTTTCACTTCATCTTGCTGGGGCATCATCAATTTTGGGAGCTATTAACTTCATCACTACTGTAATTAATATGCGG TGATCGGGACTGCGGTTAGAGCGAATCCCCCTATTTGTGTGGGCAGTAGTAATTACCGTAGTATTATTACTATTATCCCTACC GGTACTAGCAGGAGCTATTACAATGTTATTAACAGATCGAAACCTAAATACATCATTCTTTGATCCGGCTGGTGGAGGAGAT CCAATTCTATATCAACATCTATTCTGATTTTTTGGTCACCCTGAAGTTTA

\footnotetext{
$>$ WO67 A. carnosus from Jeju botanic gardens 9/9/2012 - megaBLAST < 99\% A. carnosus Nara. [Note: BLAST WO67 vs. WO24 Identities $=657 / 657(100 \%)$, WO67 vs. WO32 Identities $=655 / 655(100 \%)$, WO67 vs. WO34 Identities $=657 / 657(100 \%)$ - i.e., some other A. carnosus specimens identified from mainland Korea (Blakemore, in prep.)]. GGTCAACAAATCATAAAGATATTGGAACTTTATATTTTATCTTGGGAATCTGGGCAGGAATAATTGGTGCCGGTATGAGACT ACTTATTCGAATCGAGCTCAGGCAACCGGGCTCCTTTCTGGGAAGGGACCAACTATATAACACAATTGTAACAGCACACGC ATTCCTTATAATTTTCTTTCTAGTTATACCAGTATTCATTGGGGGATTTGGAAATTGGCTACTACCACTCATACTGGGAACAC CGGACATAGCATTTCCACGCCTAAACAACATGAGATTCTGGCTACTGCCCCCATCACTAATCTTACTAGTATCTTCCGCCGC AGTAGAGAAAGGAGCAGGTACAGGATGAACAGTATATCCCCCCCTAGCAAGAAATATTGCTCATGCGGGGCCATCAGTAGA CCTGGCAATTTTCTCACTCCACTTAGCTGGGGCATCCTCAATTTTGGGGGCCATTAACTTTATTACTACAGTAATTAATATGC GCTGGTCTGGACTACGTCTAGAGCGAATCCCGCTATTTGTATGAGCAGTAGTAATTACTGTCGTACTACTATTACTATCATTA CCAGTTCTTGCCGGAGCTATTACAATACTACTAACAGACCGAAACCTAAATACATCATTCTTCGACCCAGCGGGAGGGGGG GACCCAATTCTGTACCAACACCTATTTTGATTTTTTGGTCACCCTGAAGTTTA
}

\footnotetext{
$>$ WO68 Drawida iucn H megaBLAST closest to unidentified Japanese Moniligastridae < 81\%. BLAST WO68 vs. WO69 "D. a. seogwipo"Identities $=686 / 709$ (97\%); WO68 vs. WO46? (or rather vs. WO45) mixed and resampled as w24 "D. $a$. halla" Identities=625/650 (96\%), i.e., likely different taxa. GGTCAACAAATCATAAAGATATTGGAACTTTATATTTCATTTTAGGTGTGTGGGCAGGTATAATTGGTGCTGGTATGAGTCT TTTGATTCGGATTGAACTAAGACAGCCTGGCTCTTTTTTGGGGAGTGATCAGCTCTATAATACTATTGTTACTGCTCATGCTT TCGTTATAATTTTTTTTCTTGTAATACCTGTATTTATTGGTGGATTCGGAAATTGACTTCTTCCTCTTATATTAGGAGCACCGG ATATAGCATTCCCACGTCTTAATAATCTGAGATTTTGACTATTGCCCCCAGCATTAATTCTTTTAGTTTCTTCGGCAGCAGTG GAAAAAGGTGCTGGTACTGGGTGAACAGTATACCCGCCGCTAGCAAGAAACCTGGCTCACGCCGGTCCCTCAGTTGATTTA GCAATTTTTTCTCTTCATTTAGCAGGGGCATCTTCAATTTTGGGCTCTATTAATTTTATTACAACTGTAATTAATATACGATGA TCTGGGATAAATGTAGAACGAATTCCACTATTTGTTTGAGGTGTAGTTATTACTGTAGTATTACTTTTACTATCTCTTCCAGTT TTAGCAGGGGCAATTACTATGCTTTTGACAGATCGAAATCTGAATACGTCTTTCTTCGATCCTGCCGGCGGTGGTGACCCAA TTTTGTACCAGCACTTATTTTGATTTTTTGGTCACCCTGAAGTTTA
} 
$>$ WO69 Drawida a. seogwipo H - megaBLAST unidentified Japanese Moniligastridae <81\%. BLAST WO69 vs. WO45 (=w24 D. a. halla) Identities $=642 / 650(99 \%)$ i.e., perhaps part of the sub-sp complex; cf. WO70.

GGTCAACAAATCATAAAGATATTGGAACTTTATATTTTATTTTACGTGTGTGGGCAGGTATAATTGGTGCTGGTATAAGTCTT TTGATTCGGATTGAATTAAGACAGCCTGGCTCATTTTTGGGAAGCGATCAGCTCTATAATACTATTGTTACTGCTCATGCTTT CGTTATAATTTTTTTTCTTGTAATACCTGTATTTATTGGTGGCTTCGGAAATTGACTTCTTCCTCTTATATTAGGGGCTCCGGA TATAGCATTCCCACGTCTTAATAATCTGAGATTTTGGCTATTGCCCCCAGCATTAATTCTTTTAGTTTCTTCGGCAGCAGTGG AAAAAGGTGCTGGTACTGGGTGAACAGTATACCCGCCGCTAGCAAGCAACCTGGCTCACGCCGGTCCCTCAGTTGATTTAG CAATTTTTTCTCTTCATTTAGCAGGAGCGTCTTCAATTTTGGGCTCTATTAATTTTATTACAACTGTAATTAATATGCGATGAT CTGGGATAAATGTAGAGCGAATTCCACTATTTGTTTGAGGTGTAGTTATTACTGTAGTATTACTTTTATTATCTCTTCCGGTTT TAGCGGGAGCAATTACTATGCTTTTGACAGATCGAAATCTGAATACGTCTTTCTTCGATCCTGCCGGCGGTGGAGATCCAAT TTTGTATCAGCACTTATTTTGATTTTTTGGTCACCCTGAAGTTTA

$>$ WO70 Drawida sp.1 - megaBLAST unidentified Japanese Moniligastridae <91\%. BLAST WO70 vs. WO69 D. a. seogwipo Identities $=702 / 709(99.0 \%)$; WO70 vs. w24 D. a. halla Identities $=647 / 658(98.3 \%)$.

GGTCAACAAATCATAAAGATATTGGAACTTTATATTTTATTTTAGGTGTGTGGGCAGGAATAATTGGTGCTGGTATAAGTCT TTTGATTCGGATTGAATTAAGACAGCCTGGCTCTTTTTTGGGAAGCGATCAGCTCTATAATACTATTGTTACTGCTCATGCTT TCGTTATAATTTTTTTTCTTGTAATACCTGTATTTATTGGTGGCTTCGGAAATTGACTTCTTCCTCTTATATTAGGGGCTCCGG ATATAGCATTCCCACGTCTTAATAATCTGAGATTTTGGCTATTGCCCCCAGCATTAATTCTTTTAGTTTCTTCGGCAGCAGTG GAAAAAGGTGCTGGTACTGGGTGAACAGTATACCCGCCGCTAGCAAGCAACCTGGCTCACGCTGGTCCCTCAGTTGATTTA GCAATTTTTTCTCTTCATTTAGCAGGAGCGTCTTCAATTTTGGGCTCTATTAATTTTATTACAACTGTAATTAATATGCGATGA TCTGGGATAAATGTGGAGCGAATTCCACTATTTGTTTGAGGTGTAGTTATTACTGTAGTATTACTTTTATTATCTCTTCCGGTT TTAGCGGGGGCAATTACTATGCTTTTGACAGATCGAAATCTGAATACGTCTTTCTTCGATCCTGCCGGCGGTGGAGACCCAA TTTTGTATCAGCACTTATTTTGATTTTTTGGTCACCCTGAAGTTTA

> WO71 Drawida a. seogwipo P1 - BLAST WO69 vs. WO71 - Identities=708/709 (99.9\%), i.e., same taxon. GGTCAACAAATCATAAAGATATTGGAACTTTATATTTTATTTTAGGTGTGTGGGCAGGTATAATTGGTGCTGGTATAAGTCTT TTGATTCGGATTGAATTAAGACAGCCTGGCTCATTTTTGGGAAGCGATCAGCTCTATAATACTATTGTTACTGCTCATGCTTT CGTTATAATTTTTTTTCTTGTAATACCTGTATTTATTGGTGGCTTCGGAAATTGACTTCTTCCTCTTATATTAGGGGCTCCGGA TATAGCATTCCCACGTCTTAATAATCTGAGATTTTGGCTATTGCCCCCAGCATTAATTCTTTTAGTTTCTTCGGCAGCAGTGG AAAAAGGTGCTGGTACTGGGTGAACAGTATACCCGCCGCTAGCAAGCAACCTGGCTCACGCCGGTCCCTCAGTTGATTTAG CAATTTTTTCTCTTCATTTAGCAGGAGCGTCTTCAATTTTGGGCTCTATTAATTTTATTACAACTGTAATTAATATGCGATGAT CTGGGATAAATGTAGAGCGAATTCCACTATTTGTTTGAGGTGTAGTTATTACTGTAGTATTACTTTTATTATCTCTTCCGGTTT TAGCGGGAGCAATTACTATGCTTTTGACAGATCGAAATCTGAATACGTCTTTCTTCGATCCTGCCGGCGGTGGAGATCCAAT TTTGTATCAGCACTTATTTTGATTTTTTGGTCACCCTGAAGTTTA

>w1 Jeju \#1 trip lumbricid tumid 9(D. attemsi?) from site \#6 redo of WM10 - nil result.

$>$ w4 Re-do of WM11 A. arx from Sunrise Peak above beach $\cdots$ BLAST nothing better than $<86 \%$ Metaphire sieboldi from Japan or Amynthas spp., i.e., new species to GenBank.

BLAST w4 vs. WM11 Identities $=649 / 650(99.9 \%)$ - OK confirms original result.

BLAST $w 4$ vs. w5 Identities $=552 / 657$ (84\%), i.e., different species.

BLAST w4 vs. w6 Identities $=517 / 617(84 \%)$, i.e., different species.

BLAST $w 4$ vs. WO40 (w20) Identities $=552 / 657(84 \%)$, i.e., different species.

BLAST w4 vs. w21 Identities $=630 / 655(96.2 \%)$, i.e. close but different taxa.

AACCCTTTATTTCATTTTAGGTATCTGAGCTGGGATAATTGGAGCAGGTATGAGACTCCTTATTCGAGTTGAGCTAAGACAA CCAGGATCATTCCTAGGTAGAGACCAACTCTATAATACTATTGTTACAGCACACGCATTTTTAATAATTTTCTTTCTAGTAAT ACCAGTATTTATTGGGGGATTTGGAAACTGACTTTTACCCCTAATACTGGGGACACCAGATATAGCATTCCCACGACTAAAT AATATGAGATTTTGATTACTCCCCCCATCACTTATTTTATTAGTATCATCTGCTGCCGTTGAAAAAGGGGCAGGGACAGGAT GAACAGTATATCCACCCCTTGCAAGAAATATTGCACATGCTGGGCCATCTGTAGATCTTGCAATTTTCTCCCTTCACTTGGCG GGGGCATCATCAATTCTGGGGGCGATCAACTTTATCACCACTGTAATTAATATACGTTGATCAGGTTTACGATTAGAGCGAA TCCCACTATTTGTCTGGGCAGTCGTAATTACTGTGGTACTACTACTCCTATCCCTACCTGTGCTAGCGGGAGCCATTACAATA CTATTAACAGATCGAAATCTAAATACATCCTTCTTCGACCCGGCCGGGGGAGGGGATCCAATCCTCTACCAACACCTATTT

$>$ w5 re-do of WM12. Metaphire haenyeo $\mathrm{H}$ sp. nov from Machair.

megaBLAST $<93 \%$ Chinese herbal medicine as for other samples above.

BLASTn w5 vs. WM12 Identities $=657 / 657(100 \%)$ QED.

BLASTn w5 vs. W20 M. valhalla Identities $=651 / 658$ (98.9\%), i.e., similar samples/sub-species?

AACACTATATTTTATTTTGGGTATTTGAGCTGGAATAATTGGAGCCGGTATAAGACTACTTATTCGAATTGAGCTAAGACAG CCTGGATCATTTCTAGGAAGAGATCAACTATATAATACAATTGTAACTGCTCACGCATTCGTAATAATTTTCTTTCTGGTAAT ACCAGTATTTATTGGGGGCTTCGGAAACTGGCTACTCCCCCTAATACTTGGGACACCAGACATAGCTTTCCCACGACTAAAT AATATAAGTTTCTGACTTCTACCACCCTCACTTATTCTACTAGTATCATCCGCGGCAGTTGAAAAGGGTGCAGGAACAGGGT GAACAGTATATCCCCCACTAGCAAGAAATATTGCCCACGCTGGACCTTCAGTAGATTTGGCAATCTTTTCACTTCATCTTGCT GGGGCATCATCAATTTTGGGAGCTATTAATTTCATCACTACTGTAATTAATATGCGGTGATCGGGACTGCGGTTAGAGCGAA 
TCCCACTATTTGTGTGAGCAGTAGTAATTACCGTAGTATTACTACTACTATCCCTACCGGTACTAGCAGGGGCTATTACAATG TTATTAACAGATCGAAACCTAAATACATCATTTTTTGATCCGGCTGGTGGAGGAGATCCAATTCTATATCAACACCTATTC

\begin{abstract}
> w6 "Metaphire cf. quelparta"? Sanbangsan Temple big green damaged Metaphire (redo of WM13 \& WO11). megaBLAST < 93\% Chinese herbal medicine as for other samples above $\cdots$ BLAST w6 vs. w5 Identities $=599 / 618(97 \%)$ as before for WM13, so probably different (sub-)species?

GGAGCCGGTATAAGATTACTTATTCGAATTGAGMTAAGACAACCTGGATCATTTCTAGGAAGAGATCAACTATATAATACA ATTGTAACTGCTCACGCATTCGTAATAATTTTCTTTCTGGTAATACCAGTATTTATTGGGGGTTTCGGAAACTGGCTACTCCC CCTAATACTCGGGACACCAGACATAGCTTTCCCACGACTAAATAATATAAGATTCTGACTTCTGCCACCCTCACTTATTCTAT TGGTGTCATCCGCCGCAGTTGAAAAAGGTGCAGGAACAGGGTGGACAGTATATCCCCCACTAGCAAGAAATATTGCCCACG CTGGACCTTCAGTAGACCTGGCAATCTTTTCACTTCATCTTGCTGGGGCATCATCAATTTTGGGAGCTATTAATTTCATCACT ACTGTAATTAATATGCGGTGATCGGGACTGCGGTTAGAGCGAATCCCCCTATTTGTGTGAGCAGTAGTAATTACCGTAGTAT TACTACTACTATCCCTACCGGTACTAGCAGGGGCTATTACAATATTATTAACAGATCGAAACCTAAATACATCATTCTTTGAT CCAGCTGGTGGAGGAGATCCAATTCTATATCAACACCTATTC
\end{abstract}

\begin{abstract}
> w17 Drawida cf. koreana Jeju \#2 June 2012 (redo of WO44).
megaBLAST $<80 \%$ for unidentified Glossoscolecidae - this possibly indicating a new moniligastrid. W17 vs. "WO44"=491/647 (76\%) confirming WO44 was mixed, probably with WO45 "Eisenia cf. japonica" from the same locality

GACTTTATATTTTATTTTAGGTGTGTGAGCTGGTATAATTGGTGCTGGAATAAGCCTACTTATTCGAATTGAGTTAAGGCAGC CTGGGGCATTTCTAGGAAGTGATCAGCTATATAACACTATTGTTACTGCACATGCTTTTATTATAATTTTCTTTCTAGTAATG CCTGTTTTTATTGGTGGTTTTGGAAATTGGCTGCTACCACTAATGTTAGGGGCACCAGATATAGCTTTTCCACGGTTAAACAA TTTAAGGTTTTGACTCTTACCACCCGCACTAATTTTATTAGTGTCTTCCGCAGCTGTAGAAAAGGGTGCTGGTACTGGTTGAA CTGTATACCCCCCGCTAGCTAGAAACTTAGCTCATGCGGGCCCTTCTGTTGATTTGGCTATTTTTTCGTTACATTTAGCTGGT GCATCTTCCATTTTGGGCGCTATTAACTTTATCACAACTGTTGTAAATATGCGATGATCTGGAATGCATTTGGAGCGAATTCC TTTATTTGTGTGAGGCGTAGCTATTACTGTTGTATTACTTTTACTTTCTCTGCCAGTTTTAGCAGGCGCGATTACAATGCTTCT GACAGATCGTAATCTTAACACTTCCTTTTTCGACCCAGCTGGAGGAGGAGATCCAATTTTATACCAACATTTGTTC
\end{abstract}

>w18a Jeju \#2 Eisenia japonica (redo of WO45) BLAST 99\% Ischnura asiatica (a dragonfly from Japan)! Same as w10, w12, w15-16, w19, w22-23, w26, w28 and w40. All contamination from the genetics laboratory.

$>$ w18b Jeju \#2 Eisenia japonica (redo of WO45). BLAST 93\% for Eisenia japonica from Japan. This $2^{\text {nd }}$ try by NICEM had good result. w18b vs "WO44" Identities=651/651 (100\%), i.e., sample "WO44" was mixed in the original lab with WO45 that was E. japonica.

AACTCTATACTTCATTCTCGGGGTTTGAGCTGGTATAGTGGGCGCCGGTATAAGACTTCTTATTCGAATCGAACTAAGCCAA CCGGGAGCTTTTCTAGGGAGAGACCAATTATACAACACAATTGTGACAGCACATGCATTTGTAATAATCTTCTTTCTAGTAA TACCTGTATTTATCGGGGGTTTTGGAAATTGACTTCTACCCCTTATATTAGGAGCTCCTGATATAGCCTTCCCGCGGCTAAAT AACATAAGATTTTGACTTTTACCCCCATCCCTTATCCTTCTAGTATCTTCTGCCGCTGTCGAAAAAGGCGCGGGGACGGGAT GAACAGTTTATCCCCCCCTATCAAGAAATCTTGCTCATGCGGGCCCCTCAGTAGACCTAGCTATTTTTTCTCTACACTTAGCA GGAGCTTCTTCAATTCTGGGGGCTATCAACTTTATTACCACAGTTATTAATATACGATGAAGCGGATTACGTTTAGAGCGAA TCCCCCTATTTGTATGAGCCGTAGTTATCACAGTAGTACTCTTACTCCTATCCCTACCAGTCCTTGCAGGAGCCATTACCATA TTATTAACAGACCGAAACCTTAATACCTCATTCTTTGACCCTGCAGGAGGCGGAGACCCTATCTTATATCAACATCTTTTT

>w20 Metaphire valhalla redo of WO40 BLAST < 94\% for some Megascolecidae "Chinese herbal medicine" (as before for WO11, WM12, WO66).

BLASTn w20 vs. WM12 (w5) Jeju1 \#5 Metaphire from Machair - 650/657 (99\%) i.e., similar or same species.

AACACTATATTTTATTTTGGGTATTTGAGCCGGAATAATTGGAGCCGGTATAAGACTACTTATTCGAATTGAGCTAAGACAG CCTGGATCATTTCTAGGAAGAGATCAACTATATAATACAATTGTAACTGCTCACGCATTCGTAATAATTTTCTTTCTGGTAAT ACCAGTATTTATTGGGGGCTTCGGAAACTGGCTACTCCCCCTAATACTTGGGACACCAGACATAGCTTTCCCACGACTAAAC AATATAAGTTTCTGACTTCTACCCCCCTCACTTATTCTATTAGTATCATCCGCGGCAGTTGAAAAGGGTGCAGGAACAGGGT GAACAGTATATCCCCCACTAGCAAGAAATATTGCCCACGCTGGACCTTCAGTAGATTTAGCAATCTTTTCACTTCATCTTGCT GGGGCATCATCAATTTTGGGAGCTATTAATTTCATCACTACTGTAATTAATATGCGGTGATCGGGACTGCGGTTAGAGCGAA TCCCACTATTTGTGTGAGCAGTAGTAATTACCGTAGTATTACTTCTACTATCCCTACCGGTACTAGCAGGAGCTATTACAATG TTATTAACAGATCGAAACCTAAATACATCATTTTTTGATCCGGCTGGTGGAGGAGATCCAATTCTATATCAACACCTATTC

$>$ w21 Amynthas aucklandis (redo of WO41). BLAST <86\% for M. sieboldi or M. agrestis from Japan. BLAST w21 vs. w20 M. q. valhalla Identities: 544/655 (83\%), i.e., different taxa.

AACCCTTTATTTCATTTTAGGTATTTGAGCTGGGATAATTGGAGCAGGTATGAGACTCCTTATTCGAGTTGAGCTAAGACAA CCAGGATCATTCCTAGGTAGAGACCAACTCTATAATACTATTGTTACAGCACACGCATTTTTAATAATTTTCTTTCTAGTAAT ACCAGTATTTATTGGAGGATTTGGAAATTGACTTTTACCCCTAATACTGGGGACACCAGATATAGCATTTCCACGACTAAAT AATATGAGATTTTGATTGCTCCCCCCATCACTTATTTTATTAGTGTCATCTGCTGCTGTTGAAAAGGGGGCGGGAACAGGAT GAACAGTATATCCACCCCTTGCAAGAAATATTGCACATGCTGGGCCATCTGTAGATCTTGCAATTTTCTCCCTTCACTTGGCC GGGGCATCATCAATTCTTGGGGCCATCAACTTTATCACCACTGTAATTAATATACGCTGATCAGGTTTACGATTAGAACGAA TCCCACTATTTGTGTGGGCAGTCGTAATTACTGTGGTACTGCTACTTCTATCCCTACCTGTGCTAGCGGGAGCCATTACAATA CTATTAACAGATCGAAACCTAAATACATCCTTCTTCGATCCAGCAGGGGGCGGGGACCCAATCCTCTACCAGCACCTA 
$>$ w22 Amynthas sp (redo of WO42). Nil - contaminated in lab.

$>$ w23 Amynthas simplex (redo of WO43). Nil result - contaminated in lab.

$>$ w24 Drawida a. halla $\mathrm{H}$ (redo of WO46). BLAST <81\% for some unidentified Moniligastridae from Japan. w24 vs. WO45 "Eisenia japonica from Mt Halla" - 650/650 (100\%) shows that WO45 was mixed with original WO45. BLAST w24 vs. WO68 D. a. iucn Identities $=633 / 658(96 \%)$, i.e. probably different taxa. w24 vs. WO69 D. a. seogwipo Identities $=650 / 658(98.8 \%)$ i.e. a close match but morphological differences indicative.

AACTTTATATTTCATTTTAGGTGTGTGGGCAGGTATAATTGGTGCTGGTATAAGTCTTTTGATTCGGATTGAATTAAGACAGC CTGGCTCATTTTTGGGAAGCGATCAGCTCTATAATACTATTGTTACTGCTCATGCTTTCGTTATAATTTTTTTTCTTGTAATAC CTGTATTTATTGGTGGCTTCGGAAATTGACTTCTTCCTCTTATACTAGGGGCTCCGGATATAGCATTCCCACGTCTTAATAAT CTGAGATTTTGGCTATTGCCCCCAGCATTAATTCTTTTAGTTTCTTCGGCAGCAGTGGAAAAAGGTGCTGGTACTGGGTGAA CAGTATACCCACCGCTAGCAAGCAACCTGGCTCACGCCGGTCCCTCAGTTGATTTAGCAATTTTTTCTCTTCATTTAGCAGGA GCGTCTTCAATTTTGGGCTCAATTAATTTTATTACAACTGTAATTAATATGCGATGATCTGGGATAAATGTAGAGCGAATTCC ACTATTTGTTTGAGGTGTAGTTATTACTGTAGTATTACTTTTATTATCTCTTCCGGTTTTAGCGGGAGCAATTACTATGCTTTT GACAGATCGAAATCTGAATACGTCTTTCTTCGATCCTGCCGGTGGTGGAGACCCAATTCTGTATCAGCACTTATTT

$>$ w25 A. corticis Mt Halla temple (redo of WO47). megaBLAST 99-100\% A. corticis as expected.

AACTCTATACTTCATTTTAGGAATTTGAGCCGGAATAATTGGGGCTGGAATAAGTCTTCTTATTCGAATCGAATTAAGACAA CCTGGGTCATTCCTGGGAAATGACCAACTTTATAACACAATTGTAACAGCACATGCATTCTTAATAATTTTTTTTCTAGTAAT ACCAGTATTTATTGGTGGTTTTGGTAATTGACTATTACCACTTATATTGGGGACTCCAGATATAGCATTTCCACGCCTAAATA ATATAAGATTCTGACTATTACCCCCCTCACTCATCTTATTAGTCTCTTCTGCAGCAGTGGAAAAAGGTGCAGGAACAGGATG AACAGTTTACCCCCCACTAGCAAGTAACATTGCGCATGCTGGACCATCAGTAGATCTGGCAATTTTCTCACTACACTTAGCA GGGGCATCATCAATTCTAGGTGCAATCAATTTTATTACTACAGTAATTAATATACGATGATCAGGCCTACGACTAGAACGAA TTCCCCTATTTGTATGAGCAGTAGTAATTACCGTAGTTCTACTACTTCTATCATTACCAGTACTAGCCGGTGCTATCACAATA CTATTAACAGATCGAAACCTTAATACATCCTTCTTTGATCCAGCAGGAGGGGGAGACCCAATTTTATATCAACACCTATTT

> w26 A. micronarius Mt Halla Temple (redo of WO48). megaBLAST 100\% A. micronarius from Japan. This was $2^{\text {nd }}$ try by NICEM. BLAST WO1 vs. w26 Identities=655/655 (100\%), i.e., =A. micronarius from NIBR.

AACTTTATACTTCATCTTAGGGATCTGAGCCGGAATAATTGGTGCCGGAATAAGATTACTCATTCGAGTCGAATTAAGACAA CCCGGACCATTTCTAGGTAGGGATCAACTATACAATACAATTGTAACCGCACACGCATTCTTAATAATCTTCTTTCTAGTAAT GCCAGTATTTATTGGAGGATTTGGAAATTGATTACTACCTCTAATGTTGGGAACACCAGATATAGCATTCCCACGTCTAAAT AACATGAGATTTTGATTATTACCTCCGTCACTTATTTTACTCGTTTCATCTGCAGCCGTAGAAAAAGGAGCGGGAACAGGGT GAACAGTATACCCCCCGCTAGCAAGAAACATTGCCCATGCCGGGCCATCAGTAGACCTTGCAATTTTCTCACTACACTTAGC AGGAGCCTCCTCAATTTTAGGGGCAATTAACTTTATTACTACAGTAATTAACATGCGATGATCGGGACTACGCTTAGAACGA ATTCCCCTATTCGTATGGGCAGTCGTAATCACCGTAGTATTACTTCTTCTATCACTACCAGTATTAGCGGGAGCCATTACAAT ACTTCTTACAGACCGAAACCTTAACACATCGTTCTTTGATCCAGCAGGTGGGGGGGACCCTATTCTATACCAGCACCTGTTT

$>$ w27 A. kamitai (=maculosus) from Sambangsan stream (redo of WO51). megaBLAST <90\% A. corticis/diffringens i.e. something different! [Originally thought to be A. gracilis].

BLAST w27 vs. WO50 Identities $=619 / 620(99.9 \%)$ i.e. WO50/51 were mixed.

AACTCTATATTTCATTTTAGGAATTTGAGCCGGAATAATTGGAGCAGGGATAAGCCTACTTATCCGAATTGAATTAAGACAA CCAGGATCATTCCTTGGAAGTGATCAACTATATAACACAATTGTAACAGCACATGCATTCTTAATAATCTTTTTTCTAGTGAT ACCAGTATTTATTGGTGGATTTGGAAACTGATTATTACCCCTTATATTAGGGACTCCAGACATAGCATTCCCTCGACTAAAC AATATGAGATTTTGACTATTGCCACCCTCACTGATCTTACTAGTCTCATCTGCCGCAGTAGAAAAAGGTGCAGGGACAGGGT GGACAGTTTACCCACCCCTAGCAAGAAATATCGCACATGCGGGACCATCAGTAGATCTTGCAATTTTCTCACTACACTTAGC AGGAGCATCATCAATCTTGGGAGCAATTAATTTTATTACCACAGTAATTAATATACGGTGATCTGGGCTACGACTAGAACGA ATTCCACTATTTGTCTGAGCAGTGGTAATTACCGTAGTTTTACTACTTTTATCACTACCAGTACTAGCCGGAGCTATTACAAT ACTACTAACAGATCGAAACCTTAATACATCATTCTTTGACCCAGCA

$>$ w28 A. tralfamadore/masatakae from Cheyonji (redo of WO52). Contaminated in the lab.

$>$ w28b A. tralfamadore/masatakae from Cheyonji (redo of WO52). megaBLAST 100\% A. robustus from Japan, or $99 \%$ for "A. triastriatus" and "A. hawayanus hawayanus" from China. This was $2^{\text {nd }}$ try from NICEM. WO2 A. tralfamadore vs. w28 Identities= $608 / 647$ (94\%), i.e., different species.

BLAST WO35 vs. w28b Identities $=657 / 657(100 \%)$ i.e. $=$ A. masatakae QED.

AACCTTATACTTTATCCTGGGAATTTGAGCAGGAATAATTGGTGCAGGAATAAGACTACTTATTCGTATTGAATTAAGACAA CCGGGATCCTTCCTTGGTAGAGACCAACTATATAATACAATTGTAACAGCACATGCATTCTTAATAATCTTCTTTCTAGTAAT GCCAGTATTTATTGGTGGATTCGGAAACTGGCTACTGCCCCTAATACTTGGAACCCCAGATATGGCGTTCCCCCGATTAAAT AACATAAGATTTTGACTACTTCCACCATCCCTAATTTTATTAGTAAGATCGGCCGCGGTAGAAAAAGGAGCGGGCACAGGTT GGACAGTTTACCCACCCCTAGCAAGAAATATAGCACATGCCGGCCCTTCAGTAGACCTTGCAATTTTCTCACTCCATCTTGC AGGTGCCTCATCAATTCTTGGAGCTATTAACTTTATCACAACAGTAATTAACATGCGATGATCTGGGCTACGTCTAGAACGA ATCCCACTATTTGTATGAGCAGTAGTGATCACCGTAGTACTATTATTATTATCCCTACCTGTACTGGCTGGGGCAATTACCAT ATTACTAACGGATCGAAACCTTAATACATCATTCTTTGACCCAGCAGGAGGGGGGGACCCAGTTCTATACCAACACCTATTT 
$>$ w29 A. tralfamadore/masatakae small (redo of WO53). BLAST 93-94\% for the same triumvirate as w28. w28b vs. w29 Identities= 615/658 (93\%) i.e., different species.

WO2 vs. w29 Identities $=647 / 650(99 \%)$ i.e. probably same as $A$. tralfamadore.

WO35 vs. w29 Identities $=614 / 657$ (93\%) i.e. different to A. masatakae.

AACCCTATACTTTATTCTAGGAATTTGAGCAGGAATAATTGGTGCAGGAATAAGACTACTTATTCGTATTGAGTTAAGACAA CCTGGATCCTTCCTTGGTAGGGACCAGCTATATAATACAATTGTAACAGCACATGCATTTTTAATAATCTTCTTTCTAGTAAT GCCAGTATTTATTGGTGGATTCGGAAACTGGTTACTACCCCTAATACTTGGAACCCCAGATATGGCGTTCCCTCGATTAAAT AACATAAGATTTTGACTACTCCCGCCATCATTAATTTTATTGGTAAGATCTGCCGCGGTAGAAAAGGGGGCTGGCACAGGTT GAACAGTTTACCCACCCTTAGCAAGAAATATAGCACATGCCGGTCCTTCAGTAGACCTTGCAATTTTCTCACTTCATCTTGCA GGTGCCTCGTCAATTCTTGGAGCTATTAACTTTATCACAACAGTAATTAACATACGATGATCTGGGTTACGTCTAGAACGAA TCCCACTATTTGTGTGAGCAGTAGTAATCACTGTAGTACTATTACTATTGTCCCTACCTGTACTGGCAGGGGCAATTACCATA TTACTAACAGATCGAAACCTCAATACWTCATTCTTTGATCCAGCAGGAGGSSGAGATCCAATCCTATACCAACACCTATTT

>w30 A. tralfamadore/masatakae paler(redo of WO54). BLAST 93-94\% for the same triumvirate as W28..ditto W29 vs. w30 Identities= $655 / 658(99 \%)$ i.e. same or similar species.

w30 vs. WO2 Identities $=650 / 650(100 \%)$, i.e., same as A. tralfamadore QED.

w30 vs. W29 Identities=647/650 (99\%), i.e., same (w29 has sampling error "SS" and "W").

AACCCTATACTTTATTCTAGGAATTTGAGCAGGAATAATTGGTGCAGGAATAAGACTACTTATTCGTATTGAGTTAAGACAA CCTGGATCCTTCCTTGGTAGGGACCAGCTATATAATACAATTGTAACAGCACATGCATTTTTAATAATCTTCTTTCTAGTAAT GCCAGTATTTATTGGTGGATTCGGAAACTGGTTACTACCCCTAATACTTGGAACCCCAGATATGGCGTTCCCTCGATTAAAT AACATAAGATTTTGACTACTCCCGCCATCATTAATTTTATTGGTAAGATCTGCCGCGGTAGAAAAGGGGGCTGGCACAGGTT GAACAGTTTACCCACCCTTAGCAAGAAATATAGCACATGCCGGTCCTTCAGTAGACCTTGCAATTTTCTCACTTCATCTTGCA GGTGCCTCGTCAATTCTTGGAGCTATTAACTTTATCACAACAGTAATTAACATACGATGATCTGGGTTACGTCTAGAACGAA TCCCACTATTTGTGTGAGCAGTAGTAATCACTGTAGTACTATTACTATTGTCCCTACCTGTACTGGCAGGGGCAATTACCATA TTACTAACAGATCGAAACCTCAATACATCATTCTTTGATCCAGCAGGAGGGGGAGATCCAATCCTATACCAACACCTATTT

\begin{abstract}
$>$ w31 A. gracilis insularum (redo of WO55). megaBLAST=100\% "Amynthas gracilis" GenBank vouchers (AB542484.1 \& 542485.1) specimens were both from Ogasawara Islands, on Chichijima and Hahajima; whereas Identities=623/637 (98\%) for two specimens of "Amynthas gracilis" (AB542589.1 \& 542491.1) from Japanese mainland. Therefore, my conclusion is this is a new sub-species or species comparable to parts of A. gracilis gracilis.

AACCCTATATTTTATTCTAGGAATTTGAGCCGGAATAATTGGAGCTGGAATAAGGCTACTTATTCGAATTGAACTCAGACAG CCGGGATCGTTTCTGGGAAGAGATCAACTATATAATACAATTGTAACAGCTCATGCATTTGTAATAATTTTCTTTCTGGTAAT ACCAGTATTCATTGGTGGATTTGGAAACTGACTACTACCTCTAATGCTGGGTACACCAGACATAGCATTTCCGCGACTTAAT AATATAAGATTTTGACTACTCCCCCCGTCACTTATCTTACTAGTAAGATCCGCGGCCGTTGAAAAGGGGGCGGGAACTGGAT GAACAGTATATCCCCCGCTGGCAAGAAATATTGCACATGCTGGTCCATCAGTAGATCTAGCAATCTTCTCACTACACTTGGC AGGAGCATCATCTATTCTTGGGGCCATTAACTTTATTACAACTGTAATTAATATACGATGATCTGGATTACGGCTAGAACGA ATCCCCCTATTTGTATGGGCCGTAGTAATTACTGTAGTACTTCTACTATTATCTCTACCTGTACTAGCCGGAGCTATTACTAT ATTATTAACAGATCGAAACCTTAACACATCATTCTTTGATCCCGCTGGAGGTGGAGACCCTATTCTATATCAACACCTATTT
\end{abstract}

$>$ w32 Metaphire cf. agrestis (redo of WO56) ? nil result.

$>$ w33 A corticis saeseum (redo of WO57). BLAST <99-100\% A. corticis from Japan that has this Note: "The organism belongs to Amynthas heteropoda, presently a junior synonym of Amynthas corticis". This then is a allopatric and morphological sub-species that has recently diverged and is thus undetected by mitochondrial change.

w33 vs. w25 (redo of WO47) Identities $=614 / 658(93 \%)$ i.e., potentially different species.

w33 vs. w59 A. corticis from Incheon, Identities=611/654 (93\%), i.e., ditto.

AACCCTATACTTCATTTTAGGAATTTGAGCCGGAATAATTGGGGCAGGAATAAGTCTTCTTATTCGAATCGAATTAAGACAA CCAGGATCATTCCTGGGAAGTGACCAACTATATAACACAATTGTAACAGCACATGCATTTTTAATAATTTTTTTTCTAGTAAT ACCAGTATTTATTGGTGGCTTTGGTAACTGATTATTACCACTTATATTAGGAACTCCAGATATAGCATTCCCACGCCTAAATA ATATAAGATTCTGACTATTGCCTCCATCGCTCATCCTATTAGTCTCTTCTGCAGCAGTAGAAAAGGGTGCGGGAACAGGATG AACAGTATATCCCCCACTAGCAAGTAATATTGCACATGCTGGACCATCAGTAGATCTAGCAATTTTCTCACTACACTTAGCG GGGGCATCATCAATTCTAGGTGCAATCAATTTTATTACTACAGTAATTAATATACGATGATCCGGGCTACAACTGGAACGAA TTCCCCTATTTGTATGAGCAGTGGTAATCACCACAGTTTTACTACTTATATCATTACCAGTACTAGCAGGTGCTATTACAATA CTATTAACAGATCGAAACCTTAATACATCATTCTTTGATCCTGCGGGAGGGGGGGACCCAATTTTATATCAACATCTATTT

$>$ w34 M. cal. (redo of WO58). mega BLAST $=100 \%$ M. californica - OK.

AACCTTATATTTTATTCTAGGAATCTGAGCAGGGATAATCGGAGCAGGGATAAGATTACTTATTCGCATCGAACTAAGACAG CCTGGATCATTCCTTGGAAGAGACCAACTATATAATACAATTGTGACAGCACACGCATTTCTAATAATTTTCTTTCTGGTGAT ACCAGTATTTATTGGGGGATTTGGAAACTGACTTCTCCCACTAATGTTAGGAACCCCTGACATAGCGTTCCCTCGACTAAAT AACATAAGATTCTGACTACTGCCACCCTCATTAATTCTACTAGTTAGATCCGCGGCAGTAGAAAAGGGAGCAGGTACAGGA TGAACAGTGTACCCTCCACTAGCTAGAAACATAGCACACGCAGGTCCATCAGTAGATCTTGCAATTTTCTCACTACATTTAG CAGGTGCCTCATCAATTTTGGGGGCCATCAATTTCATTACCACTGTGATCAACATGCGATGATCAGGCCTACGCCTAGAGCG AATCCCACTATTCGTATGAGCCGTAGTAATCACTGTAGTACTCCTTCTACTATCACTTCCTGTGCTAGCGGGAGCAATTACTA 
TATTATTAACGGATCGAAACCTAAATACCTCATTCTTCGACCCTGCAGGTGGGGGAGACCCAATTCTGTATCAACACCTATT C

> w43 Drawida a. halla H "GM" cyst > BLAST w43 vs. w24 Identities 654/654 (100\%).

> w44 D. a. seogwipo H "GM" glands (parasites?) > BLAST w44 vs. WO69 Identities=656/657 (99\%), i.e., indicates only host tissue.

>w63 Amynthas gracilis Cheonji 13 June 2012 - on sheet $17^{\text {th }}$ Dec. 2012.

megaBLAST result Amynthas gracilis (AB542485.1) from Japan 100\%.

BLAST w63 vs. w31 Identities=650/650 (100\%). Taxa correspond.

TATATTTTATTCTAGGAATTTGAGCCGGAATAATTGGAGCTGGAATAAGGCTACTTATTCGAATTGAACTCAGACAGCCGGG ATCGTTTCTGGGAAGAGATCAACTATATAATACAATTGTAACAGCTCATGCATTTGTAATAATTTTCTTTCTGGTAATACCAG TATTCATTGGTGGATTTGGAAACTGACTACTACCTCTAATGCTGGGTACACCAGACATAGCATTTCCGCGACTTAATAATAT AAGATTTTGACTACTCCCCCCGTCACTTATCTTACTAGTAAGATCCGCGGCCGTTGAAAAGGGGGCGGGAACTGGATGAACA GTATATCCCCCGCTGGCAAGAAATATTGCACATGCTGGTCCATCAGTAGATCTAGCAATCTTCTCACTACACTTGGCAGGAG CATCATCTATTCTTGGGGCCATTAACTTTATTACAACTGTAATTAATATACGATGATCTGGATTACGGCTAGAACGAATCCCC CTATTTGTATGGGCCGTAGTAATTACTGTAGTACTTCTACTATTATCTCTACCTGTACTAGCCGGAGCTATTACTATATTATTA ACAGATCGAAACCTTAACACATCATTCTTTGATCCCGCTGGAGGTGGAGACCCTATTCTATATCAACACCTA 